\title{
A Decomposition of U.S. Business Sector TFP Growth into Technical Progress and Cost Efficiency Components
}

W. Erwin Diewert and Kevin J. Fox ${ }^{1}$

June 29, 2016

Discussion Paper 16-04,

Vancouver School of Economics,

The University of British Columbia,

Vancouver, Canada, V6T 1L4.

\begin{abstract}
One of the problems with index number methods for computing TFP growth is that during recessions, these methods show declines in TFP and this seems to imply that technical progress is negative during these periods. This is rather implausible since it implies technological regress; i.e., that that the production frontier has contracted. The paper works out a nonparametric method where one can decompose TFP growth into two components: a technical progress component (i.e., a shift in the production frontier over time) and an inefficiency component that is due to the fixity of capital and labour in the short run. The new decomposition is illustrated using the new Bureau of Economic Analysis (BEA) Integrated Macroeconomic Accounts which facilitated the construction of a set of productivity accounts for two key sectors of the US private business sector: the Corporate Nonfinancial Sector and the Noncorporate Nonfinancial Sector. The analysis sheds light on productivity growth slowdowns over the period 1960 to 2014.
\end{abstract}

\section{Key Words}

Total Factor Productivity, user costs, measures of technical progress, measures of technical and allocative inefficiency, nonparametric cost functions, nonparametric production theory, Fisher ideal indexes

\section{Journal of Economic Literature Classification Numbers}

C43, C61, C67, C82, D24, E22.

W. Erwin Diewert: School of Economics, University of British Columbia, Vancouver B.C., Canada, V6T 1Z1 and the School of Economics, UNSW Australia, Sydney 2052 (email: erwin.diewert@ubc.ca) and Kevin J. Fox: School of Economics, UNSW Australia, Sydney 2052 (email: K.Fox@unsw.edu.au). The first author gratefully acknowledges the financial support of the SSHRC of Canada, and both authors gratefully acknowledge the financial support of the Australian Research Council (DP150100830). 


\section{Introduction}

Total Factor Productivity (TFP) Growth is usually defined as aggregate output growth divided by aggregate input growth. ${ }^{2}$ Output growth and input growth are usually measured using (bilateral) index numbers. During recessions when outputs fall more rapidly than inputs, these index number measures of TFP growth usually show that productivity has declined. Since index number measures of TFP growth are often interpreted as measures of technical progress, such declines in measured productivity are somewhat embarrassing since they seem to imply technological regress; i.e., the production frontier has somehow managed to shrink.

Aiyar, Dalgaard and Moav (2008; 127) note that there are instances of lost knowledge and technical regress in pre-industrial times as, before the printing press and widespread literacy, technological knowledge was typically embodied in humans. Negative population and land productivity shocks could "induce the neglect of techniques rendered temporarily unprofitable". The transmission of these techniques to the following generations would then be lost. However, they also note (p. 126) the following in relation to the relevance of such ancient history to our modern economies.

"The phenomenon of technological regress cannot be reconciled with standard macroeconomic models of innovation (Romer 1990; Grossman and Helpman 1991; Aghion and Howitt 1992; Kortum 1997; Weitzman 1998; Olsson 2000) or adoption (Nelson and Phelps 1966; Eaton and Kortum 1999). Knowledge in these theories is conceived as a stock which may stagnate but never shrinks. Nor is this surprising; the theories are built to explain the facts of the contemporary world, which is mostly characterized by purposive $R \& D$ in rich countries, and the adoption of foreign technology by low-income countries."

Hence, in modern times, any interpretation of declines in productivity as representing technological regress is problematic for economic modelling and policy formulation. Of course, serious students of productivity measurement have long realized that factors other than technical change can explain changes in TFP; see e.g. Färe, Grosskopf, Norris and Zhang (1994), Syverson (2011) and references therein. Two of these explanatory factors are:

- Changes in technical and allocative inefficiency and

- Nonconstant returns to scale in production.

We will not be able to deal with the second explanatory factor in this paper (we will assume constant returns to scale technologies) but we will be able to decompose TFP growth into the product of an efficiency factor (a movement towards the production frontier) times a technical progress factor (an expansion of the production possibilities set for the sector of the economy under consideration). The novelty in our decomposition is that (i) it is a nonparametric method; (ii) it can be readily computed and (iii) our measure of TFP growth is quite closely related to the standard Fisher index of TFP growth. However, our method does have a drawback (in addition to assuming constant returns to scale): our methodology requires that

\footnotetext{
${ }^{2}$ See Jorgenson and Griliches (1967) or Balk (1998).

${ }^{3}$ Examples of technical regress in history include the loss of hydraulic mining technics following the decline of the Roman Empire (Wilson 2002) and the loss of boat building technologies following societal collapse in Easter Island (Diamond 2005).
} 
there be only one output. In many empirical contexts of interest this may not be a major issue, such as the standard macroeconomic context of considering the TFP growth of a country using national accounts data.

Given the recent heightened interest in U.S. productivity growth due to the measured productivity growth slowdown since $2004,{ }^{4}$ the new decomposition is applied to the new U.S. Bureau of Economic Analysis (BEA) Integrated Macroeconomic Accounts. These new accounts enabled the construction of a set of productivity accounts for two key sectors of the US private business sector: the Corporate Nonfinancial Sector and the Noncorporate Nonfinancial Sector.

Our methodology is explained in the following section, and is fairly closely related to the nonparametric production methodology developed by Farrell (1957). Sections 3 and 4 apply the methodology to our two sectors of the U.S. economy: the Corporate Financial Sector and the Noncorporate Nonfinancial Sector. Section 4 concludes. Appendix A briefly describes and lists our data set, which covers the years 1960-2014. Appendix B looks at how our methodology could be extended to the many output case.

\section{The Decomposition of TFP Growth into Technical Progress and Efficiency Components}

Our analytic framework broadly follows the cost decomposition framework explained in Section 2 of Diewert (2014) except that in the present paper, we assume a constant returns to scale best practice technology with one output. A significant innovation is that we are explicit about the determination of the relevant best practice technology, by assuming that the best practice technology production possibilities set is the convex, conical hull of past observations on the production unit up to and including the current period. ${ }^{5}$ Our approach can also be regarded as a modification of Farrell's (1957) one output, constant returns to scale nonparametric model of efficient production where we adapt his method of analysis to the time series context.

We assume that we have information on the period $\mathrm{t}$ output produced by a production unit, $\mathrm{y}^{\mathrm{t}}$ $>0$, on the period $t$ inputs used, $x^{t} \equiv\left[\mathrm{x}_{1}{ }^{\mathrm{t}}, \ldots, \mathrm{x}_{\mathrm{N}}{ }^{\mathrm{t}}\right]>0_{\mathrm{N}}$, and on the input prices faced during period $t$ by the production unit, $\mathrm{w}^{\mathrm{t}} \equiv\left[\mathrm{w}_{1}{ }^{\mathrm{t}}, \ldots, \mathrm{w}_{\mathrm{N}}{ }^{\mathrm{t}}\right]>0_{\mathrm{N}}$ for periods $\mathrm{t}=1, \ldots, \mathrm{T}$. Define the period $\mathrm{t}$ observed total input cost as $\mathrm{C}^{\mathrm{t}} \equiv \mathrm{w}^{\mathrm{t}} \cdot \mathrm{x}^{\mathrm{t}} \equiv \Sigma_{\mathrm{n}=1}{ }^{\mathrm{N}} \mathrm{W}_{\mathrm{n}}{ }^{\mathrm{t}} \mathrm{x}_{\mathrm{n}}{ }^{\mathrm{t}}$ for $\mathrm{t}=1, \ldots, \mathrm{T}$. ${ }^{6}$ If we divide each component of $x^{t}$ by the period t output level $y^{t}$, we obtain the vector of observed input-output coefficients for period $\mathrm{t}, \mathrm{z}^{\mathrm{t}} \equiv \mathrm{x}^{\mathrm{t}} / \mathrm{y}^{\mathrm{t}}$ for $\mathrm{t}=1, \ldots, \mathrm{T}$. If we weight the components of $\mathrm{z}^{\mathrm{t}}$ by the components of $\mathrm{w}^{\mathrm{t}}$ and sum the resulting terms, we obtain the period $\mathrm{t}$ observed unit cost $\mathrm{c}^{\mathrm{t}}$ for the production unit; i.e., we have:

(1) $\mathrm{c}^{\mathrm{t}} \equiv \mathrm{w}^{\mathrm{t}} \cdot \mathrm{z}^{\mathrm{t}}=\mathrm{w}^{\mathrm{t}} \cdot \mathrm{x}^{\mathrm{t}} / \mathrm{y}^{\mathrm{t}}=\mathrm{C}^{\mathrm{t}} / \mathrm{y}^{\mathrm{t}}$

$\mathrm{t}=1, \ldots, \mathrm{T}$.

\footnotetext{
${ }^{4}$ See Gordon (2012), Mokyr, Vickers and Ziebarth (2015), Byrne, Fernald and Reinsdorf (2016), and Syverson (2016).

${ }^{5}$ The use of nonparametric methods for estimating technical progress can be traced back to Diewert (1980b; 264) (1981), Diewert and Parkan (1983; 153-157) and Tulkens (1993; 201-206).

${ }^{6} \mathrm{We}$ assume $\mathrm{C}^{\mathrm{t}}>0$ for each $\mathrm{t}$.
} 
We assume that the production unit's period t production possibilities set can be adequately approximated by the convex ${ }^{7}$ conical free disposal hull of the period $t$ actual production vector and past production vectors that are in our sample of time series observations for the unit. ${ }^{8}$ Using this assumption, we define the period $t$ best practice unit cost function if the producer faces the nonnegative, nonzero vector of input prices $\mathrm{w}>0_{\mathrm{N}}, \mathrm{c}(\mathrm{w}, \mathrm{t})$, as follows:

$$
\text { (2) } \begin{aligned}
\mathrm{c}(\mathrm{w}, \mathrm{t}) & \equiv \min _{\lambda_{1}, \ldots, \lambda_{\mathrm{t}}}\left\{\mathrm{w} \cdot\left(\sum_{\mathrm{s}=1}{ }^{\mathrm{t}} \mathrm{x}^{\mathrm{s}} \lambda_{\mathrm{s}}\right) ; \sum_{\mathrm{s}=1}{ }^{\mathrm{t}} \mathrm{y}^{\mathrm{s}} \lambda_{\mathrm{s}} \geq 1 ; \lambda_{1} \geq 0, \ldots, \lambda_{\mathrm{t}} \geq 0\right\} \\
& =\min \left\{\mathrm{w} \cdot \mathrm{x}^{\mathrm{s}} / \mathrm{y}^{\mathrm{s}}: \mathrm{s}=1,2, \ldots, \mathrm{t}\right\} \\
& =\min \left\{\mathrm{w} \cdot \mathrm{z}^{\mathrm{s}}: \mathrm{s}=1,2, \ldots, \mathrm{t}\right\}
\end{aligned}
$$

where the second line in (2) follows from the first line since there is only one constraint in the linear programming problem defined by the first line and so the solution to the LP problem is a simple minimum of $t$ numbers.

The best practice unit cost function, $\mathrm{c}(\mathrm{w}, \mathrm{t})$, can be used to define the period $\mathrm{t}$ cost efficiency for the production unit. It will not necessarily be the case that the production unit being studied achieves the best practice level of costs in period t; i.e., the following inequalities will be satisfied:

(3) $\mathrm{c}^{\mathrm{t}}=\mathrm{w}^{\mathrm{t}} \cdot \mathrm{z}^{\mathrm{t}} \geq \mathrm{c}\left(\mathrm{w}^{\mathrm{t}}, \mathrm{t}\right)$ $\mathrm{t}=1, \ldots, \mathrm{T}$.

Thus the observed period $t$ unit cost, $c^{t}$, will be equal to or greater than the best practice minimum unit cost, $\mathrm{c}\left(\mathrm{w}^{\mathrm{t}}, \mathrm{t}\right)$. Define the cost efficiency of the production unit during period $\mathrm{t}, \mathrm{e}^{\mathrm{t}}$, as follows: ${ }^{9}$

(4) $\mathrm{e}^{\mathrm{t}} \equiv \mathrm{c}\left(\mathrm{w}^{\mathrm{t}}, \mathrm{t}\right) / \mathrm{w}^{\mathrm{t}} \cdot \mathrm{z}^{\mathrm{t}} \leq 1$

$$
\mathrm{t}=1, \ldots, \mathrm{T}
$$

where the inequalities in (4) follow from the inequalities in (3). We note that this definition of cost efficiency is equivalent to Farrell's $(1957 ; 255)$ measure of overall efficiency, which combined his measures of technical and allocative inefficiency.

Given the above definition of cost efficiency in period $\mathrm{t}$, we can define an index of the change in the production unit's cost efficiency going from period $t-1$ to $t$ as follows:

(5) $\varepsilon^{\mathrm{t}} \equiv \mathrm{e}^{\mathrm{t}} / \mathrm{e}^{\mathrm{t}-1}=\left[\mathrm{c}\left(\mathrm{w}^{\mathrm{t}}, \mathrm{t}\right) / \mathrm{c}^{\mathrm{t}}\right] /\left[\mathrm{c}\left(\mathrm{w}^{\mathrm{t}-1}, \mathrm{t}-1\right) / \mathrm{c}^{\mathrm{t}-1}\right]$.

Thus if $\varepsilon^{t}>1$, then the cost efficiency of the production unit has improved going from period $\mathrm{t}-1$ to $\mathrm{t}$ whereas it has fallen if $\varepsilon^{\mathrm{t}}<1$.

\footnotetext{
${ }^{7}$ The convexity assumption is not required in the one output case. If we assume that the best practice technology can be represented by the conical Free Disposal Hull (FDH) of past observations, then the corresponding best practice unit cost function is still defined by (2). For references to the FDH approach to nonparametric production theory, see the pioneering contributions by Tulkens (1986) (1993) and his coauthors and the subsequent papers by Diewert and Fox (2014) (2016a). Our approach can be viewed as applying cost data to Tulkens (1993; 201-206) Sequential FDH measurement of efficiency and local technical progress approach.

${ }^{8}$ This approximation will become more adequate for the later observations in our sample.

${ }^{9}$ This definition follows Balk $(1998 ; 28)$. Note that $\mathrm{e}^{1}$ is equal to unity.
} 
We now use the best practice unit cost function to define a family of measures of technical progress, $\tau(\mathrm{w}, \mathrm{t})$; i.e., measures of expansion in the production possibilities set going from period $t-1$ to $t$. For a reference vector of input prices $w>0_{N}$, define $\tau(w, t)$ as follows: ${ }^{10}$

(6) $\tau(w, t) \equiv c(w, t-1) / c(w, t)$.

In the regulatory literature, it is quite common to specify technical progress in terms of downward shifts in the cost function over time. Thus in definition (6), we pick a reference vector $\mathrm{w}$ and use the best practice technology of period 0 to calculate the minimum cost of producing one unit of output at the input prices $\mathrm{w}$ using the period $\mathrm{t}-1$ and $\mathrm{t}$ best practice technologies at those times. This gives rise to the unit costs, $c(w, t-1)$ and $c(w, t)$, respectively. If there is positive technical progress going from period $t-1$ to $t$, then $c(w, t)$, will generally be less than $\mathrm{c}(\mathrm{w}, \mathrm{t}-1)$ and hence $\tau(\mathrm{w}, \mathrm{t})=\mathrm{c}(\mathrm{w}, \mathrm{t}-1) / \mathrm{c}(\mathrm{w}, \mathrm{t})$ will be greater than one and this measure of technical progress is the reciprocal of the degree of proportional cost reduction that results from the expansion of the underlying best practice technology sets due to the passage of time. For each choice of a reference vector of input prices w, we obtain a (possibly different) measure of exogenous cost reduction and hence of technical progress. For the period $\mathrm{t}$ measures, it is natural to choose $\mathrm{w}$ to be $\mathrm{w}^{\mathrm{t}-1}$ or $\mathrm{w}^{\mathrm{t}}$, leading to the following Laspeyres and Paasche type measures of period t technical progress:

(7) $\tau_{\mathrm{L}}^{\mathrm{t}} \equiv \tau\left(\mathrm{w}^{\mathrm{t}-1}, \mathrm{t}\right)=\mathrm{c}\left(\mathrm{w}^{\mathrm{t}-1}, \mathrm{t}-1\right) / \mathrm{c}\left(\mathrm{w}^{\mathrm{t}-1}, \mathrm{t}\right)$;

(8) $\tau_{\mathrm{P}}{ }^{\mathrm{t}} \equiv \tau\left(\mathrm{w}^{\mathrm{t}}, \mathrm{t}\right) \quad=\mathrm{c}\left(\mathrm{w}^{\mathrm{t}}, \mathrm{t}-1\right) / \mathrm{c}\left(\mathrm{w}^{\mathrm{t}}, \mathrm{t}\right)$;

$\mathrm{t}=2,3, \ldots, \mathrm{T}$

$\mathrm{t}=2,3, \ldots, \mathrm{T}$.

Since both of the above measures of technical progress, $\tau_{L}{ }^{t}$ and $\tau_{P}{ }^{t}$, are equally representative, a single estimate of technical progress should be set equal to a symmetric average of these two estimates. We choose the geometric mean as our preferred symmetric average ${ }^{11}$ and thus our preferred summary measure of technical progress going from period $t-1$ to $t$ is the following Fisher type index of technical progress, $\tau^{\mathrm{t}}$; for $\mathrm{t}=2, \ldots, \mathrm{T}^{12}$

(9) $\tau^{\mathrm{t}} \equiv\left[\tau_{L}^{\mathrm{t}} \tau_{\mathrm{P}}^{\mathrm{t}}\right]^{1 / 2}$.

We now turn our attention to measures of the effects on best practice unit cost of input price change. We use the period t best practice unit cost function $\mathrm{c}(\mathrm{w}, \mathrm{t})$ in order to define a family of input price indexes going from period $\mathrm{t}-1$ to period $\mathrm{t}, \beta\left(\mathrm{w}^{\mathrm{t}-1}, \mathrm{w}^{\mathrm{t}}, \mathrm{s}\right)$, as follows: ${ }^{13}$

\footnotetext{
${ }^{10}$ This type of cost based measure of technical progress can be traced back to Salter (1960). Balk (1998; 58) defined a family of indexes similar to that defined by (6) by using the best practice total cost function in place of the best practice unit cost function. The definitions (6)-(13) are specializations (to the case of one output) of the cost function based definitions used by Diewert $(2011 ; 181-182)(2012 ; 223-225)$ to decompose total cost growth into explanatory factors using a reference best practice cost function. What is missing in Diewert's decompositions is the change in cost efficiency term $\varepsilon^{t}$ defined by (5).

${ }^{11}$ This will ensure that the resulting measure of technical progress satisfies the time reversal property; i.e., if we reverse the role of time and recalculate the measure of technical progress, we obtain the reciprocal of the original measure when we take the geometric average.

${ }^{12}$ Fisher (1922) defined his ideal index as the geometric mean of the corresponding Laspeyres and Paasche indexes and noted that the resulting index satisfied the time reversal test.

${ }^{13}$ If the number of outputs is equal to one, then the family of indexes defined by (10) reduces to a Konüs (1939) true cost of living index family in the case of homothetic preferences where $\mathrm{c}(\mathrm{w}, \mathrm{t})$ is the consumer's unit utility expenditure function using period t preferences and output is interpreted as a utility level. Shephard (1953) developed this theory of input price indexes for the case of a constant returns to scale production function.
} 
(10) $\beta\left(\mathrm{w}^{\mathrm{t}-1}, \mathrm{w}^{\mathrm{t}}, \mathrm{s}\right) \equiv \mathrm{c}\left(\mathrm{w}^{\mathrm{t}}, \mathrm{s}\right) / \mathrm{c}\left(\mathrm{w}^{\mathrm{t}-1}, \mathrm{~s}\right)$;

$\mathrm{t}=2,3, \ldots, \mathrm{T}$.

Thus the input price index $\beta\left(\mathrm{w}^{\mathrm{t}-1}, \mathrm{w}^{\mathrm{t}}, \mathrm{s}\right)$ defined by (10) is equal to the (hypothetical) unit cost $\mathrm{c}\left(\mathrm{w}^{\mathrm{t}}, \mathrm{s}\right)$ of producing one unit of output when the production unit faces the period $\mathrm{t}$ observed vector of input prices $\mathrm{w}^{\mathrm{t}}$, divided by the unit $\operatorname{cost} \mathrm{c}\left(\mathrm{w}^{\mathrm{t}}, \mathrm{s}\right)$ of producing one unit of output when the production unit faces the period $t-1$ observed vector of input prices $\mathrm{w}^{\mathrm{t}-1}$, where in both cases, the production unit has access to the best practice technology of period s. Hence for each choice of technology, we obtain a (possibly different) input price index.

It is natural to choose $\mathrm{s}$ to be equal to either $\mathrm{t}-1$ or $\mathrm{t}$. Thus for these choices of $\mathrm{s}$, we obtain the following Laspeyres and Paasche type input price indexes, $\beta_{\mathrm{L}}{ }^{\mathrm{t}}$ and $\beta_{\mathrm{P}}{ }^{\mathrm{t}}$, that measure input price change going from period $\mathrm{t}-1$ to $\mathrm{t}$ :

(11) $\beta_{\mathrm{L}}{ }^{\mathrm{t}} \equiv \beta\left(\mathrm{w}^{\mathrm{t}-1}, \mathrm{w}^{\mathrm{t}}, \mathrm{t}-1\right)=\mathrm{c}\left(\mathrm{w}^{\mathrm{t}}, \mathrm{t}-1\right) / \mathrm{c}\left(\mathrm{w}^{\mathrm{t}-1}, \mathrm{t}-1\right)$;

$\mathrm{t}=2,3, \ldots, \mathrm{T}$

(12) $\beta_{\mathrm{P}}{ }^{\mathrm{t}} \equiv \beta\left(\mathrm{w}^{\mathrm{t}-1}, \mathrm{w}^{\mathrm{t}}, \mathrm{t}\right) \quad=\mathrm{c}\left(\mathrm{w}^{\mathrm{t}}, \mathrm{t}\right) / \mathrm{c}\left(\mathrm{w}^{\mathrm{t}-1}, \mathrm{t}\right)$;

$\mathrm{t}=2,3, \ldots, \mathrm{T}$.

Since both input price indexes, $\beta_{\mathrm{L}}{ }^{\mathrm{t}}$ and $\beta_{\mathrm{P}}{ }^{\mathrm{t}}$, are equally representative, a single estimate of input price change should be set equal to a symmetric average of these two estimates. We again choose the geometric mean as our preferred symmetric average and thus our preferred overall measure of input price growth going from period $\mathrm{t}-1$ to $\mathrm{t}$ is the following Fisher type theoretical input price index, $\beta^{\mathrm{t}}$ :

(13) $\beta^{t} \equiv\left[\beta_{L}{ }^{t} \beta_{P}^{t}\right]^{1 / 2}$

$$
\mathrm{t}=2,3, \ldots, \mathrm{T}
$$

The input price index $\beta^{\mathrm{t}}$ can be used to deflate the (total) cost ratio going from period $\mathrm{t}$ to $\mathrm{t}-1$, $\mathrm{C}^{\mathrm{t}} / \mathrm{C}^{\mathrm{t}-1}$, in order to obtain the following implicit input quantity index going from period $\mathrm{t}-1$ to $\mathrm{t}, \gamma^{\mathrm{t}}$ :

(14) $\gamma^{\mathrm{t}} \equiv\left[\mathrm{C}^{\mathrm{t}} / \mathrm{C}^{\mathrm{t}-1}\right] / \beta^{\mathrm{t}}$

$\mathrm{t}=2,3, \ldots, \mathrm{T}$.

Following Jorgenson and Griliches (1967), Total Factor Productivity Growth going from period $t-1$ to $t$, $\mathrm{TFP}_{\mathrm{G}}{ }^{\mathrm{t}}$, can be defined as (one plus) output growth, $\mathrm{y}^{\mathrm{t}} / \mathrm{y}^{\mathrm{t}-1}$, divided by (one plus) input growth, $\gamma^{\mathrm{t}}$ :

(15) $\mathrm{TFPG}^{\mathrm{t}} \equiv\left[\mathrm{y}^{\mathrm{t}} / \mathrm{y}^{\mathrm{t}-1}\right] / \gamma^{\mathrm{t}}$;

$\mathrm{t}=2,3, \ldots, \mathrm{T}$.

We can use the above definitions to show that TFP growth is equal to the product of cost efficiency growth times technical progress; i.e., we can show that $\mathrm{TFPG}^{\mathrm{t}}=\varepsilon^{\mathrm{t}} \tau^{\mathrm{t}}$ where $\varepsilon^{\mathrm{t}}$ is defined by (5) and $\tau^{t}$ is defined by (9). To show this, start off with definition (15):

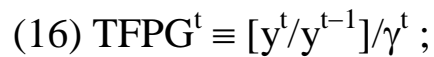

$$
\begin{aligned}
& =\left[\mathrm{y}^{\mathrm{t}} / \mathrm{y}^{\mathrm{t}-1}\right] \beta^{\mathrm{t}} /\left[\mathrm{C}^{\mathrm{t}} / \mathrm{C}^{\mathrm{t}-1}\right] \\
& =\left[\mathrm{C}^{\mathrm{t}-1} / \mathrm{C}^{\mathrm{t}}\right]\left[\mathrm{c}\left(\mathrm{w}^{\mathrm{t}}, \mathrm{t}\right) / \mathrm{c}\left(\mathrm{w}^{\mathrm{t}-1}, \mathrm{t}-1\right)\right]\left[\mathrm{c}\left(\mathrm{w}^{\mathrm{t}-1}, \mathrm{t}-1\right) / \mathrm{c}\left(\mathrm{w}^{\mathrm{t}}, \mathrm{t}\right)\right] \beta^{\mathrm{t}} \\
& =\varepsilon^{\mathrm{t}}\left[\mathrm{c}\left(\mathrm{w}^{\mathrm{t}-1}, \mathrm{t}-1\right) / \mathrm{c}\left(\mathrm{w}^{\mathrm{t}}, \mathrm{t}\right)\right] \beta^{\mathrm{t}} \\
& =\varepsilon^{\mathrm{t}}\left[\mathrm{c}\left(\mathrm{w}^{\mathrm{t}-1}, \mathrm{t}-1\right) / \mathrm{c}\left(\mathrm{w}^{\mathrm{t}}, \mathrm{t}\right)\right]\left[\beta_{\mathrm{L}}{ }^{\mathrm{t}} \beta_{\mathrm{P}}{ }^{\mathrm{t}}\right]^{1 / 2} \\
& =\varepsilon^{\mathrm{t}}\left[\mathrm{c}\left(\mathrm{w}^{\mathrm{t}-1}, \mathrm{t}-1\right) / \mathrm{c}\left(\mathrm{w}^{\mathrm{t}}, \mathrm{t}\right)\right]\left[\mathrm{c}\left(\mathrm{w}^{\mathrm{t}}, \mathrm{t}-1\right) / \mathrm{c}\left(\mathrm{w}^{\mathrm{t}-1}, \mathrm{t}-1\right)\right]^{1 / 2}\left[\mathrm{c}\left(\mathrm{w}^{\mathrm{t}}, \mathrm{t}\right) / \mathrm{c}\left(\mathrm{w}^{\mathrm{t}-1}, \mathrm{t}\right)\right]^{1 / 2}
\end{aligned}
$$




$$
\begin{aligned}
& =\varepsilon^{\mathrm{t}}\left[\mathrm{c}\left(\mathrm{w}^{\mathrm{t}}, \mathrm{t}-1\right) / \mathrm{c}\left(\mathrm{w}^{\mathrm{t}}, \mathrm{t}\right)\right]^{1 / 2}\left[\mathrm{c}\left(\mathrm{w}^{\mathrm{t}-1}, \mathrm{t}-1\right) / \mathrm{c}\left(\mathrm{w}^{\mathrm{t}-1}, \mathrm{t}\right)\right]^{1 / 2} \\
& =\varepsilon^{\mathrm{t}}\left[\tau_{\mathrm{P}}^{\mathrm{t}}\right]^{1 / 2}\left[\tau_{\mathrm{L}}^{\mathrm{t}}\right]^{1 / 2} \\
& =\varepsilon^{\mathrm{t}} \tau^{\mathrm{t}}
\end{aligned}
$$

using (11) and (12)

rearranging terms

using (7) and (8)

using (9).

As will be seen in subsequent sections of the paper, the decomposition of TFP growth defined by (16) seems to work well in practice; i.e., the time $t$ technical progress measure $\tau^{t}$ never falls below 1 (so there is never any technological regress using our methodology) and the time t measure of cost efficiency change $\varepsilon^{\mathrm{t}}$ falls below 1 during recession years as anticipated. ${ }^{14}$

Finally, it is convenient to convert the above growth decomposition into a levels decomposition. Thus we start off by setting the period 1 input level, $\mathrm{X}^{1}$, and the period 1 output level, $\mathrm{y}^{1},{ }^{15}$ equal to period 1 total cost, $\mathrm{C}^{1}$ :

(17) $X^{1}=y^{1} \equiv C^{1}$.

The period 1 TFP level TFP ${ }^{1}$, cost efficiency level $\mathrm{E}^{1}$ and technology level $\mathrm{T}^{1}$ are all set equal to 1:

(18) $\mathrm{TFP}^{1}=\mathrm{E}^{1}=\mathrm{T}^{1} \equiv 1$.

The period $t$ input level $X^{t}$, TFP level TFP ${ }^{t}$, cost efficiency level $E^{t}$ and technology level $T^{t}$ for $t$ greater than one are defined recursively as follows:

(19) $\mathrm{X}^{\mathrm{t}} \equiv \gamma^{\mathrm{t}} \mathrm{X}^{\mathrm{t}-1} ; \mathrm{TFP}^{\mathrm{t}} \equiv \mathrm{y}^{\mathrm{t}} / \mathrm{X}^{\mathrm{t}} ; \mathrm{E}^{\mathrm{t}} \equiv \varepsilon^{\mathrm{t}} \mathrm{E}^{\mathrm{t}-1} ; \mathrm{T}^{\mathrm{t}} \equiv \tau^{\mathrm{t}} \mathrm{T}^{\mathrm{t}-1}$ $\mathrm{t}=2,3 \ldots ., \mathrm{T}$.

Using the above definitions, it can be shown that we have the following decomposition of the productivity level at time $t$ into the product of the time $t$ level of cost efficiency times the time t level of technology; i.e., we have: ${ }^{16}$

(20) $\mathrm{TFP}^{\mathrm{t}}=\mathrm{y}^{\mathrm{t}} / \mathrm{X}^{\mathrm{t}}=\mathrm{E}^{\mathrm{t}} \mathrm{T}^{\mathrm{t}}$;

$\mathrm{t}=1, \ldots, \mathrm{T}$.

We will illustrate the decompositions defined by (16) and (20) using U.S. data in the following sections.

\section{TFP Decompositions for the U.S. Corporate Nonfinancial Sector}

The US Bureau of Economic Analysis (BEA), in conjunction with the Bureau of Labor Statistics (BLS) and the Board of Governors of the Federal Reserve, have developed a new set of production accounts (the Integrated Macroeconomic Accounts or IMA) for two major

\footnotetext{
${ }^{14}$ Our measure of technical progress is not perfect: in a given period, cost inefficiency could be large enough to negate the effects of actual technical progress so that our nonparametric indicator of technical progress will indicate that there is no technical progress when in fact there was technical progress.

${ }^{15}$ This implies that we are choosing a particular unit of measurement for aggregate output.

${ }^{16}$ Of course, equation (20) can be rearranged to give a decomposition of real output of the production unit in period $t, y^{t}$, into the product of the period $t$ input level $X^{t}$ times the period $t$ level of cost efficiency $E^{t}$ times the period t level of technology $\mathrm{T}^{\mathrm{t}}$. This is similar to Kohli's (1990) decomposition of nominal GDP into the product of explanatory factors. See also Fox and Kohli (1998) for a similar decomposition.
} 
private sectors of the US economy: the Corporate Nonfinancial Sector and the Noncorporate Nonfinancial Sector. The Balance Sheet Accounts in the IMA cover the years 1960-2014 but do not provide a decomposition of output, input and asset values into price and quantity components. Diewert and Fox (2016b) provided such a decomposition and we will use their data in this study. The data for the two subsectors are described and listed in Appendix A. In this section, we will use their output and input data for the U.S. Corporate Nonfinancial Sector for the 55 years 1960-2014. The year $\mathrm{t}$ output $\mathrm{y}^{\mathrm{t}}$ is real value added ${ }^{17}$ and the corresponding year $t$ value added deflator is denoted as $\mathrm{p}^{\mathrm{t}}$. The ten inputs used by this sector are labour and the services of nine types of asset, which are listed in Appendix A. The year $t$ input vector is $\mathrm{x}^{\mathrm{t}} \equiv\left[\mathrm{x}_{1}{ }^{\mathrm{t}}, \mathrm{x}_{2}{ }^{\mathrm{t}}, \ldots, \mathrm{x}_{10}{ }^{\mathrm{t}}\right]$ where $\mathrm{x}_{1}{ }^{\mathrm{t}}$ is year $\mathrm{t}$ labour input measured in billions of 1960 dollars and $\mathrm{x}_{2}{ }^{\mathrm{t}}, \ldots, \mathrm{x}_{10}{ }^{\mathrm{t}}$ are capital service inputs measured in billions of 1960 capital stock dollars. The corresponding year $\mathrm{t}$ input price vector is $\mathrm{w}^{\mathrm{t}} \equiv\left[\mathrm{w}_{1}{ }^{\mathrm{t}}, \mathrm{w}_{2}{ }^{\mathrm{t}}, \ldots, \mathrm{w}_{10}{ }^{\mathrm{t}}\right]$ for $\mathrm{t}=$ $1960, \ldots, 2014$.

It is straightforward to do the computations using the above data to compute the year $t$ cost efficiency factors $e^{t}$ defined by (4), the year $t$ change in cost efficiency factors $\varepsilon^{t}$ defined by (5), the year $t$ measures of technical progress $\tau^{\mathrm{t}}$ defined by (9), the nonparametric input price index $\beta^{\mathrm{t}}$ defined by (13), the nonparametric input quantity (or volume) index $\gamma^{\mathrm{t}}$ defined by (14) and the nonparametric Total Factor Productivity Growth factor for year t, $\mathrm{TFPG}^{\mathrm{t}} \equiv$ $\left[\mathrm{y}^{\mathrm{t}} / \mathrm{y}^{\mathrm{t}-1}\right] / \gamma^{\mathrm{t}}$ defined by (15). These growth factors are listed in Table 1 below along with the output growth factors, $\mathrm{y}^{\mathrm{t}} / \mathrm{y}^{\mathrm{t}-1}$. For comparison purposes, we also computed chained Fisher (1922) input indexes where $\gamma^{\mathrm{t}^{*}}$ denotes the year $\mathrm{t}$ Fisher chain link. ${ }^{18}$ These Fisher input indexes were then used to form a conventional Fisher TFP growth index, TFPG ${ }^{t^{*}}$, which is defined as follows:

(21) $\mathrm{TFPG}^{\mathrm{t}^{*}} \equiv\left[\mathrm{y}^{\mathrm{t}} / \mathrm{y}^{\mathrm{t}-1}\right] / \gamma^{\mathrm{t}^{*}}$

$$
\mathrm{t}=1961, \ldots, 2014
$$

The growth factors $y^{t} / y^{t-1}, \gamma^{t^{*}}$ and the Fisher Total Factor Productivity Growth index, TFPG ${ }^{t^{*}}$ are also listed in Table 1. It can be verified that the TFP growth decomposition defined by (16) in the previous section holds; i.e., for each year $t$, nonparametric TFP growth TFPG ${ }^{t}$ equals the product of cost efficiency growth $\varepsilon^{t}$ times the year $t$ cost based technical progress measure $\tau^{\mathrm{t}}$. It can also be seen when cost efficiency in year $\mathrm{t}, \mathrm{e}^{\mathrm{t}}$, is less than one, then the year $\mathrm{t}$ technical progress measure $\tau^{\mathrm{t}}$ always equals one so that there is no technical progress in years where the cost efficiency is less than one. Our nonparametric measure of technical progress $\tau^{\mathrm{t}}$ is always equal to or greater than one; i.e., our measure never indicates technological regress. Another important empirical regularity emerges from Table 1: for most years, when the year $t$ cost efficiency growth factor $\varepsilon^{t}$ is equal to one, the Fisher index measure of TFP growth, $\mathrm{TFPG}^{\mathrm{t}^{*}}$, is equal to our nonparametric measure of TFP growth, TFPG ${ }^{t}$. This follows from the fact, that for most years, the Fisher index of input growth, $\gamma^{t^{*}}$, is equal to the corresponding nonparametric index of input growth, $\gamma^{\mathrm{t}}$. Thus empirically, our nonparametric indexes of TFP growth are not all that different from ordinary Fisher indexes of TFP growth. Over the 55 years in our sample, the geometric average nonparametric rate of TFP growth TFPG $^{t}$ was $1.688 \%$ per year while the corresponding Fisher index rate of TFP

\footnotetext{
${ }^{17}$ The published data for this sector did not allow Diewert and Fox to decompose real value added into gross output and intermediate input components.

${ }^{18}$ The year $\mathrm{t}$ Fisher chain link input index is defined as $\gamma^{\mathrm{t}^{*}} \equiv\left[\left(\mathrm{w}^{\mathrm{t}-1} \cdot \mathrm{x}^{\mathrm{t}} \mathrm{w}^{\mathrm{t}} \cdot \mathrm{x}^{\mathrm{t}}\right) /\left(\mathrm{w}^{\mathrm{t}-1} \cdot \mathrm{x}^{\mathrm{t}-1} \mathrm{w}^{\mathrm{t}} \cdot \mathrm{x}^{\mathrm{t}-1}\right)\right]^{1 / 2}$.
} 
growth TFPG $^{\mathrm{t}^{*}}$ was $1.693 \%$ per year. ${ }^{19}$ These average rates of TFP growth are very large indeed!

Table 1: U.S. Corporate Nonfinancial Fisher TFP Growth TFPG ${ }^{*}$, Nonparametric TFP Growth TFPG ${ }^{t}$, Cost Efficiency Growth Factors $\varepsilon^{t}$, Technical Progress Growth Factors $\tau^{t}$, Nonparametric Input Growth Factors $\gamma^{\mathrm{t}}$, Fisher Input Growth Indexes $\gamma^{t^{*}}$, Output Growth Factors $y^{t} / y^{t-1}$ and Cost Efficiency Factors $e^{t}$

\begin{tabular}{|c|c|c|c|c|c|c|c|c|c|}
\hline Year & TFPG $^{t^{*}}$ & TFPG $^{t}$ & $\varepsilon^{t}$ & $\overline{\tau^{t}}$ & $\beta^{t}$ & $\gamma^{t}$ & $\overline{\gamma^{t^{*}}}$ & $y^{t} / y^{t-1}$ & $\mathbf{e}^{\mathbf{t}}$ \\
\hline 1961 & 1.0191 & 1.0191 & 1.0000 & 1.0191 & 1.0222 & 1.0047 & 1.0047 & 1.0238 & 1.0000 \\
\hline 1962 & 1.0484 & 0484 & 1.0000 & 1.0484 & 1.0552 & 1.0346 & 1.0346 & 1.0847 & 10000 \\
\hline 1963 & 1.0364 & 1.0364 & 1.0000 & 1.0364 & 1.0416 & 1.0244 & 1.0244 & 1.0617 & 1.0000 \\
\hline 1964 & 1.0415 & 1.0415 & 1.0000 & 1.0415 & 1.0511 & 1.0275 & 1.0275 & 1.0702 & 1.0000 \\
\hline 1965 & 1.0372 & 1.0372 & 1.0000 & 1.0372 & 1.0555 & 1.0452 & 1.0452 & 1.0841 & 1.0000 \\
\hline 1966 & 1.0217 & 1.0217 & 1.0000 & 1.0217 & 1.0515 & 1.0511 & 1.0511 & 1.0739 & 1.0000 \\
\hline 1967 & 12 & 12 & 1.0000 & 1.0012 & 1.0236 & 1.0274 & 1.0274 & 1.0287 & 1.0000 \\
\hline 1968 & 1.0291 & 1.0291 & 1.0000 & 1.0291 & 1.0611 & 1.0347 & 1.0347 & 1.0649 & 1.0000 \\
\hline 1969 & 0.9994 & 0.9995 & 0.9995 & 1.0000 & 1.0416 & 1.0405 & 1.0406 & 1.0400 & 0.9995 \\
\hline 1970 & 0.9923 & 0.9934 & 0.9934 & 1.0000 & 1.0303 & 0.9979 & 0.9990 & 0.9913 & 0.9929 \\
\hline 1971 & 1.0318 & 1.0317 & 1.0072 & 1.0244 & 1.0690 & 1.0074 & 1.0074 & 1.0394 & 1.0000 \\
\hline 1972 & & & 1.0000 & 0333 & 1.0701 & 1.0411 & 1.0411 & 1.0758 & 1.0000 \\
\hline 1973 & 1.0124 & 1.0124 & 1.0000 & 1.0124 & 1.0717 & 1.0478 & 1.0478 & 1.0607 & 1.0000 \\
\hline 1974 & 0.97 & 0.97 & 0.9742 & 1.0000 & 1.0699 & 1.0110 & 1.0113 & 0.9848 & 0.9742 \\
\hline 1975 & 5 & 31 & 1.0031 & 1.0000 & 1.1015 & 0.9828 & 0.9824 & 0.9858 & 0.9772 \\
\hline 1976 & & 1.0 & 1.0233 & 1.0203 & 1.0949 & 1.0361 & 1.0355 & 1.0819 & 1.0000 \\
\hline $197^{\prime}$ & & & 1.0000 & 0299 & 1.0882 & 1.0425 & 1.0425 & 1.0737 & 1.0000 \\
\hline 1978 & 1.01 & 1. & 1.0000 & 1.0126 & 1.0850 & 1.0517 & 1.0517 & 1.0650 & 1.0000 \\
\hline 1979 & 0. & 0. & 0.9937 & 1.0000 & 1.0752 & 1.0386 & 1.0386 & 1.0321 & 0.9937 \\
\hline 1980 & 0. & 54 & 0.9864 & 1.0000 & 1.0787 & 1.0038 & 1.0042 & 0.9902 & 0.9802 \\
\hline 1981 & & & 1.0202 & 1.0005 & 1.1085 & 1.0195 & 1.0193 & 1.0406 & 1.0000 \\
\hline 198 & 0. & 0. & 0.9929 & 1.0000 & 1.0520 & 0.9852 & 0.9858 & 0.9782 & 0.9929 \\
\hline 1082 & & & 1.0072 & 1.0219 & 1.0481 & 1.0192 & 1.0190 & 1.0490 & 1.0000 \\
\hline 198 & & & 1.0000 & 33 & 1.0704 & 1.0489 & 1.0489 & 1.0891 & 1.0000 \\
\hline 1985 & & & 00 & 1. & 87 & & 52 & 1.0463 & 1.0000 \\
\hline 108 & & & 1.0000 & 1. & 51 & 1.0149 & & 1.0261 & 1.0000 \\
\hline 198 & & & 1.0000 & 10 & 135 & 1.0283 & 1.0 & 1.0530 & 1.0000 \\
\hline 1988 & & & 1.0000 & & & & & & 1.0000 \\
\hline 1989 & 0.99 & 0.9 & 0.9941 & 1.0000 & 1.0243 & 1.0251 & 1.0251 & 1.0190 & 0.9941 \\
\hline 1990 & & & 1. & 1 & & 1.0074 & 1.0073 & 45 & 1.0000 \\
\hline 1001 & & 1. & 1.0000 & 7 & 30 & 0.9843 & 0.9843 & 0.9 & 1.0000 \\
\hline 199 & & & 1.0000 & 8 & 338 & 1.0095 & 1.0095 & 1.0305 & 1.0000 \\
\hline - & & & & & & & & & 1.0000 \\
\hline 1994 & & & 1.0000 & 31 & 37 & 1.0326 & 1.0326 & 1.0615 & 1.0000 \\
\hline 199 & & & 1.0000 & & & & 1.0324 & 1.0 & 1.0000 \\
\hline - & & & 0000 & & & & & & 1000 \\
\hline 199 & & & 1.0000 & 1. & 1.0361 & 1.0377 & 1.0377 & 1.0667 & 1.0000 \\
\hline & & & & & & & & & 1.0000 \\
\hline 1999 & & & 0 & & & 22 & & 44 & 1.0000 \\
\hline 2000 & & & & & & & & & 1.0000 \\
\hline & & & & & & & & & \\
\hline 200 & & & & & & & 46 & 1.0086 & 1.0000 \\
\hline & & & & & & & & & \\
\hline & & & & & & 98 & & & 1.0000 \\
\hline 2005 & & & & & & & & & 1.0000 \\
\hline & & & & & & & & & \\
\hline 200 & & & & & & & & & 97 \\
\hline 2008 & & & & & & & & 0.9871 & 908 \\
\hline 2009 & 0.9748 & 0.9762 & 0.9762 & 1.0000 & 0.9921 & 0.9517 & 0.9531 & 0.9290 & 0.96 \\
\hline
\end{tabular}

\footnotetext{
${ }^{19}$ These percentages are actually percentage points. The corresponding geometric average rate of growth for our nonparametric rate of input growth $\gamma^{t}$ was $1.881 \%$ per year while the corresponding Fisher index rate of input growth $\gamma^{t^{*}}$ was $1.886 \%$ per year.
} 


\begin{tabular}{llllllllll}
\hline 2010 & 1.0538 & $\mathbf{1 . 0 5 2 7}$ & $\mathbf{1 . 0 3 3 9}$ & $\mathbf{1 . 0 1 8 2}$ & $\mathbf{1 . 0 5 3 5}$ & $\mathbf{1 . 0 0 2 6}$ & $\mathbf{1 . 0 0 1 5}$ & $\mathbf{1 . 0 5 5 4}$ & $\mathbf{1 . 0 0 0 0}$ \\
$\mathbf{2 0 1 1}$ & $\mathbf{1 . 0 0 3 3}$ & $\mathbf{1 . 0 0 3 3}$ & $\mathbf{1 . 0 0 0 0}$ & $\mathbf{1 . 0 0 3 3}$ & $\mathbf{1 . 0 2 5 6}$ & $\mathbf{1 . 0 2 1 7}$ & $\mathbf{1 . 0 2 1 7}$ & $\mathbf{1 . 0 2 5 1}$ & $\mathbf{1 . 0 0 0 0}$ \\
$\mathbf{2 0 1 2}$ & $\mathbf{1 . 0 1 6 6}$ & $\mathbf{1 . 0 1 6 6}$ & $\mathbf{1 . 0 0 0 0}$ & $\mathbf{1 . 0 1 6 6}$ & $\mathbf{1 . 0 3 3 6}$ & $\mathbf{1 . 0 2 3 4}$ & $\mathbf{1 . 0 2 3 4}$ & $\mathbf{1 . 0 4 0 3}$ & $\mathbf{1 . 0 0 0 0}$ \\
$\mathbf{2 0 1 3}$ & $\mathbf{1 . 0 0 8 5}$ & $\mathbf{1 . 0 0 8 5}$ & $\mathbf{1 . 0 0 0 0}$ & $\mathbf{1 . 0 0 8 5}$ & $\mathbf{1 . 0 1 5 0}$ & $\mathbf{1 . 0 2 1 6}$ & $\mathbf{1 . 0 2 1 6}$ & $\mathbf{1 . 0 3 0 3}$ & $\mathbf{1 . 0 0 0 0}$ \\
$\mathbf{2 0 1 4}$ & $\mathbf{1 . 0 0 4 8}$ & $\mathbf{1 . 0 0 4 8}$ & $\mathbf{1 . 0 0 0 0}$ & $\mathbf{1 . 0 0 4 8}$ & $\mathbf{1 . 0 1 2 8}$ & $\mathbf{1 . 0 2 6 9}$ & $\mathbf{1 . 0 2 6 9}$ & $\mathbf{1 . 0 3 1 7}$ & $\mathbf{1 . 0 0 0 0}$ \\
\hline
\end{tabular}

Table 2: Corporate Nonfinancial Fisher TFP Levels TFP ${ }^{t^{*}}$, Nonparametric TFP Levels TFP ${ }^{t}$, Cumulated Cost Efficiency Levels $\mathbf{E}^{\mathrm{t}}$, Technology Levels $\mathbf{T}^{\mathbf{t}}$, Real Value Added $\mathbf{y}^{\mathrm{t}}$, Nonparametric Aggregate Input $\mathrm{X}^{\mathrm{t}}$ and Fisher Year $\mathbf{t}$ Input Levels $\mathrm{X}^{\mathbf{t}^{*}}$

\begin{tabular}{|c|c|c|c|c|c|c|c|}
\hline Year & TFP $^{\mathbf{t}^{*}}$ & TFP $^{t}$ & $E^{t}$ & $T^{t}$ & $\mathbf{y}^{t}$ & $\mathbf{X}^{\mathbf{t}}$ & $\mathbf{X}^{\mathbf{t}^{*}}$ \\
\hline 1960 & 1.00000 & 1.00000 & 1.00000 & 1.00000 & 255.90 & 255.90 & 255.90 \\
\hline 1961 & 1.01910 & 1.01910 & 1.00000 & 1.01910 & 262.00 & 257.10 & 257.10 \\
\hline 1962 & 1.06840 & 1.06840 & 1.00000 & 1.06840 & 284.19 & 266.00 & 266.00 \\
\hline 1963 & 1.10730 & 1.10730 & 1.00000 & 1.10730 & 301.71 & 272.47 & 272.47 \\
\hline 1964 & 1.15330 & 1.15330 & 1.00000 & 1.15330 & 322.89 & 279.97 & 279.97 \\
\hline 1965 & 1.19620 & 1.19620 & 1.00000 & 1.19620 & 350.04 & 292.62 & 292.62 \\
\hline 1966 & 1.22220 & 1.22220 & 1.00000 & 1.22220 & 375.92 & $\mathbf{3 0 7 . 5 8}$ & 307.58 \\
\hline 1967 & 1.22370 & 1.22370 & 1.00000 & 1.22370 & 386.69 & 316.00 & 316.00 \\
\hline 1968 & 1.25940 & 1.25940 & 1.00000 & 1.25940 & 411.77 & 326.97 & 326.97 \\
\hline 1969 & 1.25870 & 1.25870 & 0.99950 & 1.25940 & 428.24 & 340.21 & 340.23 \\
\hline 1970 & 1.24900 & 1.25040 & 0.99290 & 1.25940 & 424.52 & 339.51 & 339.89 \\
\hline 1971 & 1.28870 & 1.29000 & 1.00000 & 1.29000 & 441.24 & 342.03 & 342.40 \\
\hline 1972 & 1.33160 & 1.33300 & 1.00000 & 1.33300 & 474.69 & 356.11 & 356.48 \\
\hline 1973 & 1.34800 & 1.34950 & 1.00000 & 1.34950 & 503.52 & 373.12 & 373.52 \\
\hline 1974 & 1.31280 & 1.31460 & 0.97420 & 1.34950 & 495.88 & 377.21 & 377.74 \\
\hline 1975 & 1.31730 & 1.31870 & 0.97720 & 1.34950 & 488.85 & 370.70 & 371.09 \\
\hline 1976 & 1.37630 & 1.37690 & 1.00000 & 1.37690 & 528.86 & 384.10 & 384.27 \\
\hline 1977 & 1.41740 & 1.41 & 1.00000 & 1.41800 & 567.81 & 400.43 & 400.61 \\
\hline 1978 & 1.43530 & 1.43 & 1.00000 & 1.43590 & 604.69 & 421.12 & 421.31 \\
\hline 1979 & 1.42620 & 1.42680 & 0.99370 & 1.43590 & 624.08 & 437.40 & 437.59 \\
\hline 1980 & 1.40630 & 1.40750 & 0.98020 & 1.43590 & 617.97 & 439.07 & 439.44 \\
\hline 1981 & 1.43570 & 1.43 & 1.00 & 1.43660 & 643.05 & 447.62 & 447.91 \\
\hline 1982 & 1.4 & 1.42 & 0.9 & 1.43660 & 629.01 & 440.99 & 441.55 \\
\hline 1983 & 1.46640 & 1.46 & 1.00000 & 1.46800 & 659.80 & 449.46 & 449.95 \\
\hline 1984 & 1.52250 & 1.52420 & 1.00000 & 1.52420 & 718.57 & 471.44 & 471.95 \\
\hline 1985 & 1.55400 & 1.55570 & 1.00000 & 1.55570 & 751.87 & 483.31 & 483.84 \\
\hline 1986 & 1.57100 & 1.57280 & 1.00000 & 1.57280 & 771.48 & 490.53 & 491.06 \\
\hline 1987 & 1.60 & 1.6 & 1.0 & 1.61060 & 812.39 & 504.41 & 504.96 \\
\hline 1988 & 1.66390 & 1.66 & 1.00000 & 1.66570 & 862.30 & 517.68 & 518.25 \\
\hline 1989 & 1.65400 & 1.65580 & 0.99 & 1.66570 & 878.69 & 530.67 & 531.24 \\
\hline 1990 & 1.66580 & 1.66750 & 1.00000 & 1.66750 & 891.40 & 534.58 & 535.13 \\
\hline 1991 & 1.68 & 1.68 & 1.0 & 1.68540 & 886.79 & 526.17 & 526.71 \\
\hline 1992 & 1.7 & 1.72 & 1.0 & 1.72040 & 913.84 & 531.19 & 531.74 \\
\hline 1993 & 1.72480 & 1.72660 & 1.00000 & 1.72660 & 936.36 & 542.33 & 542.89 \\
\hline 1994 & 1.77320 & 1.77500 & 1.00000 & 1.77500 & 993.98 & 559.98 & 560.56 \\
\hline 1995 & 1.80050 & 1.80240 & 1.00000 & 1.80240 & 1041.97 & 578.11 & 578.71 \\
\hline 1996 & 1.8 & 1.8 & 1.0 & 1.86620 & 103.04 & 591.08 & 591.69 \\
\hline 1997 & 1.91 & 1.9 & 1.00000 & 1.9 & 1176.66 & 613.39 & 614.02 \\
\hline 1998 & 1.97770 & 1.97980 & 1.00000 & 1.97980 & 1243.46 & 628.09 & 628.74 \\
\hline 1999 & 2.02020 & 2.02230 & 1.00000 & 2.02230 & 1311.12 & 648.33 & 649.01 \\
\hline 2000 & 2.07430 & 2.07640 & 1.00000 & 2.07640 & 1382.97 & 666.04 & 666.73 \\
\hline 2001 & 2.06250 & 2.06660 & 0.99530 & 2.07640 & 1353.60 & 654.98 & 656.28 \\
\hline 2002 & 2.11280 & 2.11700 & 1.00000 & 2.11700 & 1365.17 & 644.85 & 646.14 \\
\hline 2003 & 2.18020 & 2.18450 & 1.00000 & 2.18450 & 1394.84 & 638.51 & 639.78 \\
\hline 2004 & 2.25620 & 2.26070 & 1.00000 & 2.26070 & 1457.60 & 644.75 & 646.04 \\
\hline 2005 & 2.30260 & 2.30 & 1.00000 & 2.30720 & 1505.65 & 652.58 & 653.89 \\
\hline 2006 & 2.34780 & 2.35250 & 1.00000 & 2.35250 & 1563.63 & 664.66 & 665.99 \\
\hline 2007 & 2.34690 & 2.35180 & 0.99970 & 2.35250 & 1579.29 & 671.53 & 672.93 \\
\hline 2008 & 2.32540 & 2.33090 & 0.99080 & 2.35250 & 1558.90 & 668.79 & 670.39 \\
\hline 2009 & 2.26660 & 2.27540 & 0.96720 & 2.35250 & 1448.25 & 636.47 & 638.94 \\
\hline 2010 & 2.38870 & 2.39530 & 1.00000 & 2.39530 & 1528.52 & 638.14 & 639.90 \\
\hline 2011 & 2.39660 & 2.40320 & 1.00000 & 2.40320 & 1566.84 & 651.97 & 653.77 \\
\hline
\end{tabular}




\begin{tabular}{llllllll}
\hline 2012 & 2.43640 & $\mathbf{2 . 4 4 3 1 0}$ & $\mathbf{1 . 0 0 0 0 0}$ & $\mathbf{2 . 4 4 3 1 0}$ & $\mathbf{1 6 3 0 . 0 5}$ & $\mathbf{6 6 7 . 2 0}$ & $\mathbf{6 6 9 . 0 4}$ \\
$\mathbf{2 0 1 3}$ & $\mathbf{2 . 4 5 7 1 0}$ & $\mathbf{2 . 4 6 3 9 0}$ & $\mathbf{1 . 0 0 0 0 0}$ & $\mathbf{2 . 4 6 3 9 0}$ & $\mathbf{1 6 7 9 . 4 3}$ & $\mathbf{6 8 1 . 6 2}$ & $\mathbf{6 8 3 . 5 1}$ \\
$\mathbf{2 0 1 4}$ & $\mathbf{2 . 4 6 8 8 0}$ & $\mathbf{2 . 4 7 5 6 0}$ & $\mathbf{1 . 0 0 0 0 0}$ & $\mathbf{2 . 4 7 5 6 0}$ & $\mathbf{1 7 3 2 . 7 4}$ & $\mathbf{6 9 9 . 9 3}$ & $\mathbf{7 0 1 . 8 6}$ \\
\hline
\end{tabular}

From Table 1, it can be seen that there were cost efficiencies $\mathrm{e}^{\mathrm{t}}$ below 1 for the following eleven years: 1969, 1970, 1974, 1979, 1980, 1982, 1989, 2001, 2007, 2008 and 2009. For the most part, these years were recession years for the U.S. economy. These efficiency declines were less than one percent in most years except there was a $3.28 \%$ efficiency decline for this sector in $2009 .{ }^{20}$ Thus in general, cost inefficiencies are a transitory phenomenon which show up during recessions but which vanish when the economy recovers from the recession. Nevertheless, the cost inefficiency factors $e^{t}$ provide a rough and ready numerical estimate of the costs of a recession. During the Great Recession, this cost was significant and it illustrates the importance of macroeconomic stability in preventing recessions.

Recall that equations (17)-(19) in the previous section converted the growth decomposition defined by (16) into the levels decomposition $\mathrm{TFP}^{\mathrm{t}} \equiv \mathrm{y}^{\mathrm{t}} / \mathrm{X}^{\mathrm{t}}=\mathrm{E}^{\mathrm{t}} \mathrm{T}^{\mathrm{t}}$ defined by (20) where $\mathrm{TFP}^{\mathrm{t}}$ is the level of nonparametric TFP in year $t, y^{t}$ is output in year $t, X^{t}$ is the level of nonparametric input in year $t, E^{t}$ is the cumulated level of cost efficiency in year $t$ (which is equal to $\mathrm{e}^{\mathrm{t}}$, the cost efficiency factor defined by (4) above) and $\mathrm{T}^{\mathrm{t}}$ is the cumulated level of technical progress for year $\mathrm{t}$. Define $\mathrm{X}^{\mathrm{t}^{*}}$ as the Fisher chained index level of input for year $\mathrm{t}$ and define the Fisher index level of TFP in year $t$ as $\mathrm{TFP}^{\mathrm{t}^{*}} \equiv \mathrm{y}^{\mathrm{t}} / \mathrm{X}^{\mathrm{t}^{*}}$. The Fisher input and productivity level series, $\mathrm{X}^{\mathrm{t}^{*}}$ and $\mathrm{TFP}^{\mathrm{t}^{*}}$, are also listed in Table 2 for comparison purposes. It can be seen that these indexes are very close to their nonparametric counterparts, $\mathrm{X}^{\mathrm{t}}$ and $\mathrm{TFP}^{\mathrm{t}}$. It can also be seen that the technology level series, $\mathrm{T}^{\mathrm{t}}$, is also very close to $\mathrm{TFP}^{\mathrm{t}}$ and $\mathrm{TFP}^{\mathrm{t}^{*}}$.

The nonparametric TFP levels TFP ${ }^{t}$, cumulated cost efficiency factors $E^{t}$ (equal to the year $t$ cost efficiency factor $e^{t}$ ) and the year $t$ level of technology $T^{t}$ are plotted in Figure 1 . It can be seen that except for recession years $t$ when $\mathrm{e}^{\mathrm{t}}$ is less than 1 , the year $t$ nonparametric TFP level $\mathrm{TFP}^{\mathrm{t}}$ is equal to the corresponding level of technology index $\mathrm{T}^{\mathrm{t}}$. During the years $t$ when $\mathrm{e}^{\mathrm{t}}$ is less than 1, TFP $^{t}$ falls below the technology index, $T^{t}$, since $T_{F P}^{t} / T^{t}$ is equal to $e^{t}$.

It can be seen that the rate of technical change in the Corporate Nonfinancial sector slowed down between the years 1973-1982, grew rapidly during 1983-1988, slowed down during 1989-1993, grew rapidly during 1994-2006 and then experienced a major slowdown 20062009 and then grew modestly during 2009-2014.

\section{Figure 1: Corporate Nonfinancial Sector TFP Levels TFP ${ }^{t}$, Cumulated Cost Efficiency Factors $E^{t}$ and Levels of Technology $T^{t}$}

\footnotetext{
${ }^{20}$ Note that the output decline in 2009 was $7.10 \%$ while the nonparametric input decline was $4.83 \%$. Thus during recessions when demand drops unexpectedly, many inputs are quasi-fixed and cannot drop at the same rate, leading to a TFP decline. Thus during recession years, the observed output and input combination generally ends up in the interior of the production possibilities set.
} 


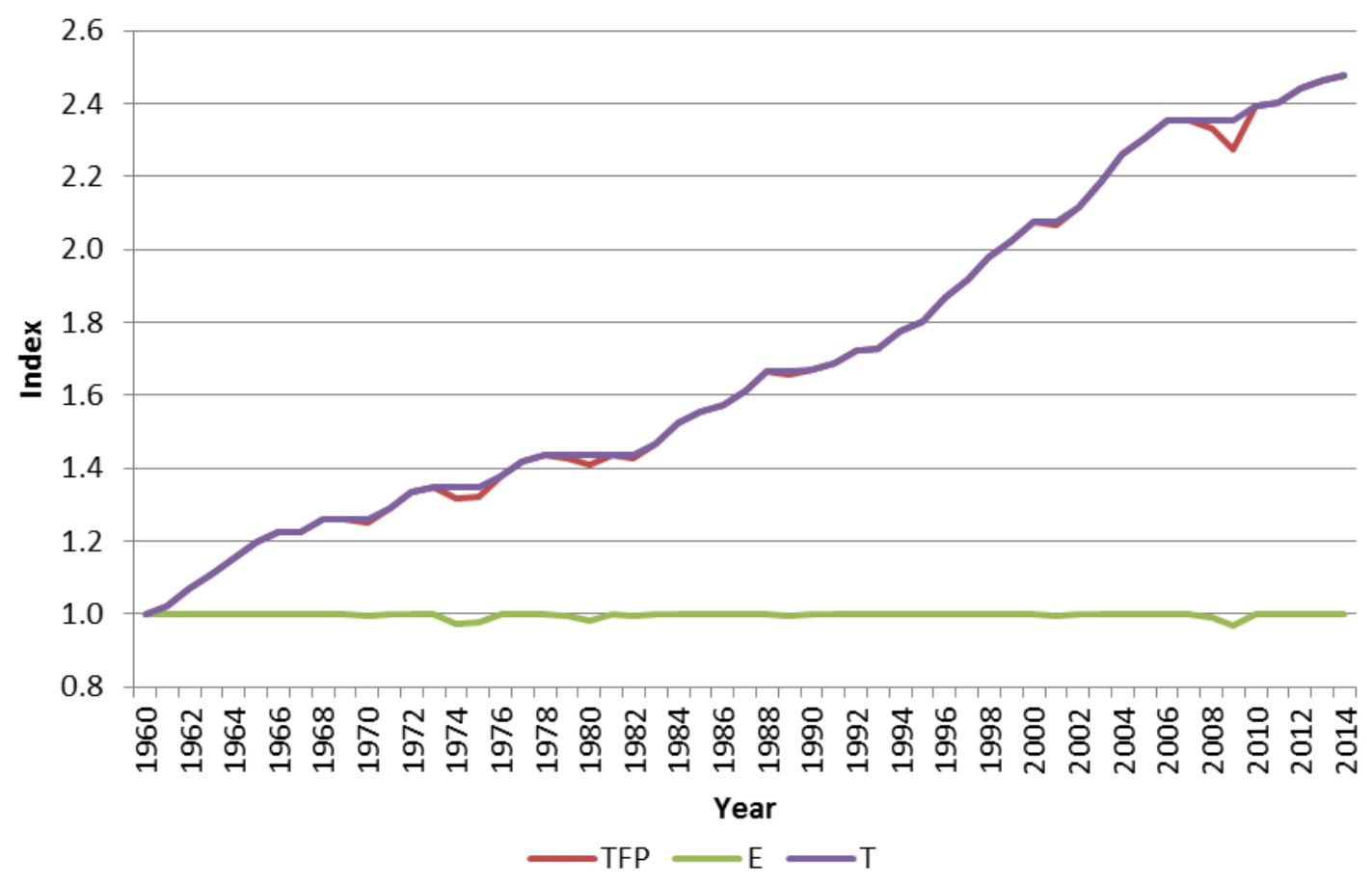

\section{TFP Decompositions for the U.S. Noncorporate Nonfinancial Sector}

In this section, we will use the Diewert and Fox (2016b) output and input data for the U.S. Noncorporate Nonfinancial Sector for the 55 years 1960-2014. We use the same notation that was used in the previous section. Now the year $t$ output $y^{t}$ is Noncorporate Nonfinancial real value added and the corresponding year $t$ value added deflator is denoted as $p^{t}$. The fifteen inputs used by this sector are labour and the services of fourteen types of asset, which are listed in Appendix A. The year $\mathrm{t}$ input vector is $\mathrm{x}^{\mathrm{t}} \equiv\left[\mathrm{x}_{1}{ }_{1}^{\mathrm{t}}, \mathrm{x}_{2}{ }^{\mathrm{t}}, \ldots, \mathrm{x}_{15}{ }^{\mathrm{t}}\right]$ where $\mathrm{x}_{1}{ }^{\mathrm{t}}$ is year $\mathrm{t}$ labour input measured in billions of 1960 dollars and $\mathrm{x}_{2}{ }^{\mathrm{t}}, \ldots, \mathrm{x}_{15}{ }^{\mathrm{t}}$ are capital service inputs measured in billions of 1960 capital stock dollars. The corresponding year $t$ input price vector is $\mathrm{w}^{\mathrm{t}} \equiv$ $\left[\mathrm{w}_{1}{ }^{\mathrm{t}}, \mathrm{w}_{2}{ }^{\mathrm{t}}, \ldots, \mathrm{w}_{15}{ }^{\mathrm{t}}\right]$ for $\mathrm{t}=1960, \ldots, 2014$.

Again, it is straightforward using the above data to compute the year $t$ cost efficiency factors $\mathrm{e}^{\mathrm{t}}$ defined by (4), the year $\mathrm{t}$ change in cost efficiency factors $\varepsilon^{\mathrm{t}}$ defined by (5), the year $\mathrm{t}$ measures of technical progress $\tau^{\mathrm{t}}$ defined by (9), the nonparametric input price index $\beta^{\mathrm{t}}$ defined by (13), the nonparametric input quantity (or volume) index $\gamma^{t}$ defined by (14) and the nonparametric Total Factor Productivity Growth factor for year $t, \mathrm{TFPG}^{\mathrm{t}} \equiv\left[\mathrm{y}^{\mathrm{t}} / \mathrm{y}^{\mathrm{t}-1}\right] / \gamma^{\mathrm{t}}$ defined by (15). These growth factors are listed in Table 3 along with the output growth factors, $y^{t} / y^{t-1}$. For comparison purposes, we also computed chained Fisher input indexes where $\gamma^{t^{*}}$ denotes the year $t$ Fisher chain link. These Fisher input indexes were then used to form the Fisher TFP growth index, TFPG ${ }^{\mathrm{t}^{*}}$, which was defined by (21) in the previous section. The output and

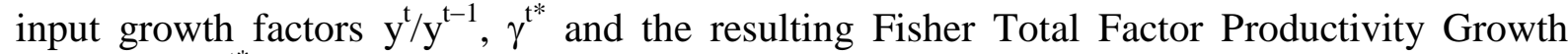
index, TFPG $^{\mathrm{t}^{*}}$ are also listed in Table 3 .

Table 3: U.S. Noncorporate Nonfinancial Fisher TFP Growth TFPG ${ }^{t^{*}}$, Nonparametric TFP Growth TFPG ${ }^{t}$, Cost Efficiency Growth Factors $\varepsilon^{t}$, Technical Progress Growth Factors $\tau^{t}$, 
Nonparametric Input Growth Factors $\gamma^{\mathrm{t}}$, Fisher Input Growth Indexes $\gamma^{\mathrm{t}^{*}}$, Output Growth Factors $\mathbf{y}^{\mathrm{t}} / \mathbf{y}^{\mathrm{t}-1}$ and Cost Efficiency Factors $\mathrm{e}^{\mathrm{t}}$

\begin{tabular}{|c|c|c|c|c|c|c|c|c|c|}
\hline Year & TFPG $^{t^{*}}$ & TFPG $^{t}$ & $\varepsilon^{t}$ & $\tau^{\mathrm{t}}$ & $\beta^{t}$ & $\gamma^{\mathrm{t}}$ & $\gamma^{t^{*}}$ & $\mathbf{y}^{\mathrm{t} / \mathbf{y}^{\mathrm{t}-1}}$ & $\mathrm{e}^{\mathbf{t}}$ \\
\hline 1961 & 1.0270 & 1.0270 & 1.0000 & 1.0270 & 1.0435 & 0.9834 & 0.9834 & 1.0099 & 1.0000 \\
\hline 1962 & 1.0315 & 0315 & 1.0000 & 1.0315 & 1.0462 & 0.9905 & 0.9905 & 1.0217 & 1.0000 \\
\hline 1963 & 1.0243 & 0243 & 1.0000 & 1.0243 & 1.0360 & 0.9898 & 0.9898 & 1.0138 & 1.0000 \\
\hline 1964 & 1.0317 & 1.0317 & .0000 & 1.0317 & 1.0490 & 1.0013 & 1.0013 & 1.0330 & 1.0000 \\
\hline 1965 & 1.0389 & 1.0389 & 1.0000 & 1.0389 & 1.0596 & 0.9975 & 0.9975 & 1.0363 & 1.0000 \\
\hline 1966 & 1.0332 & 1.0332 & 1.0000 & 1.0332 & 1.0649 & 1.0005 & 1.0005 & 1.0337 & 1.0000 \\
\hline 1967 & & 1.0000 & 1.0000 & 1.0000 & 1.0317 & 0.9945 & 0.9947 & 0.9945 & \\
\hline 1968 & 1.0162 & 1.0162 & 1.0000 & 1.0162 & 1.0622 & 0.9924 & 0.9924 & 1.0085 & 1.0000 \\
\hline 1969 & 1.0033 & 1.0033 & 1.0000 & 1.0033 & 1.0472 & 1.0059 & 1.0059 & 1.0092 & 1.0000 \\
\hline 1970 & 0.9998 & 1.0002 & 1.0000 & 1.0002 & 1.0395 & 0.9962 & 0.9966 & 0.9964 & 1.0000 \\
\hline 1971 & 1.0153 & 1.0153 & 1.0000 & 1.0153 & 1.0631 & 0.9965 & 0.9965 & 1.0117 & 1.0000 \\
\hline 1972 & & 7 & 1.0000 & 1.0397 & 1.0896 & 1.0137 & 1.0137 & 1.0539 & 1.0000 \\
\hline 1973 & 1.0818 & 1.0818 & 1.0000 & 1.0818 & 1.1292 & 1.0353 & 1.0353 & 1.1199 & 1.0000 \\
\hline 1974 & 0. & 0.9404 & 0.9404 & 1.0000 & 1.0290 & 1.0227 & 1.0231 & 0.9617 & 0.9404 \\
\hline 1975 & 0.9780 & 0.9782 & 0.9782 & 1.0000 & 1.0789 & 0.9930 & 0.9932 & 0.9713 & 0.9199 \\
\hline 1976 & 0.9992 & 0.9990 & 0.9990 & 1.0000 & 1.0860 & 1.0071 & 1.0068 & 1.0061 & 0.9189 \\
\hline 1977 & 0.9921 & 19 & 0.9919 & 1.0000 & 1.0752 & 1.0148 & 1.0145 & 1.0065 & 0.9114 \\
\hline 1978 & 1.0298 & 91 & 1.0291 & 1.0000 & 1.1026 & 1.0276 & 1.0269 & 1.0575 & 0.9380 \\
\hline 1979 & 0.97 & 03 & 0.9703 & 1.0000 & 1.0848 & 1.0290 & 1.0292 & 0.9985 & 0.9101 \\
\hline 1980 & 0.9706 & 14 & 0.9714 & 1.0000 & 1.0362 & 1.0145 & 1.0152 & 0.9854 & 0.8841 \\
\hline 1981 & 0.9 & 83 & 0.9883 & 1.0000 & 1.0841 & 1.0048 & 1.0052 & 0.9930 & 0.8737 \\
\hline 198 & & & 0.9735 & 1.0000 & 1.0364 & 1.0071 & 1.0091 & 0.9804 & 0.8506 \\
\hline 198 & & (o & 0.9678 & 1.0000 & 1.0407 & 1.0162 & 1.0180 & 0.9835 & 0.8232 \\
\hline 198 & & & 1.1144 & 1.0000 & .1287 & 1.0254 & 1.0239 & 1.1427 & 0.9174 \\
\hline 1985 & & 1. & 1. & 1.0000 & & & & & \\
\hline 198 & & & 8 & 1.0000 & 91 & 1.0103 & 1.0130 & 1.0414 & 0.9627 \\
\hline 198 & & & 0.9858 & 1.0000 & 0342 & 1.0144 & 1.0140 & 1.0000 & 0.9491 \\
\hline 108 & & & 32 & 1.0000 & 28 & & 1.0153 & 1.0389 & 0.9711 \\
\hline 198 & & 0.9914 & 0.9914 & 1.0000 & 1.0444 & 1.0193 & 1.0212 & 1.0105 & 0.9627 \\
\hline 1990 & & & 0.9929 & 1.0000 & & & & 1.0014 & 0.9558 \\
\hline 199 & 0 . & 0 & 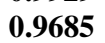 & 00 & 37 & 1.0005 & 1.0046 & 0.9690 & 0.9257 \\
\hline 1992 & & & 1. & 1.0000 & 24 & 0.9860 & 0.9867 & 1.0508 & 0.9865 \\
\hline 100 & & & 0 . & 00 & & & & & 0.9786 \\
\hline 199 & & 1. & 1.0219 & 67 & 05 & 1.0118 & 1.0124 & 1.0408 & 1.0000 \\
\hline 199 & & & & 00 & & 1.0 & 1.0094 & 0.9958 & 0.9865 \\
\hline 199 & & & 7 & 27 & 88 & 1.0088 & 1.0088 & 1.0254 & 1.0000 \\
\hline 1997 & & & 1. & 92 & 28 & 1.0193 & 1.0193 & 87 & 1.0000 \\
\hline & & & & & & & & & 1.0000 \\
\hline 1999 & & & 00 & 2 & & 86 & 086 & 81 & 1.0000 \\
\hline 200 & & & & & & & & & 1.0000 \\
\hline 200 & & & 00 & 9 & 60 & 1.0809 & 1.0809 & 1.1035 & 1.0000 \\
\hline 200 & & & 1. & & & & 1. & 81 & 1.0000 \\
\hline & & & & & & & & & \\
\hline 200 & & & & & & & & & 1.0000 \\
\hline 200 & & & & & & & & & 1.0000 \\
\hline 20 & & & & & & & & & 1.0000 \\
\hline 200 & & & 0. & 00 & 0.9975 & 1.0301 & 1.0299 & 1.0030 & 0.9737 \\
\hline & & & & & & & & & \\
\hline 2009 & & & & & & & & & 0.9748 \\
\hline 2 & & & & & & & & & 0.9779 \\
\hline & & & & & & & & & \\
\hline 2012 & & & & & & & & & \\
\hline 2013 & & & & & & & & & \\
\hline 2014 & 1.0034 & 1.0034 & 1.0000 & 1.0034 & 1.0270 & 1.0170 & 1.0170 & 1.0205 & 1.0000 \\
\hline
\end{tabular}

It can be verified that the TFP growth decomposition defined by (16) holds; i.e., for each year $\mathrm{t}$, nonparametric TFP growth TFPG ${ }^{\mathrm{t}}$ equals the product of $\cos$ efficiency growth $\varepsilon^{\mathrm{t}}$ times the 
year $t$ cost based technical progress measure $\tau^{t}$. As in the previous section, it can also be seen when cost efficiency in year $t, e^{t}$, is less than one, then the year $t$ technical progress measure $\tau^{t}$ always equals one so that there is no technical progress in years where the cost efficiency is less than one.

Over the 55 years in our sample, the geometric average nonparametric rate of TFP growth TFPG $^{\mathrm{t}}$ was $1.210 \%$ per year while the corresponding Fisher index rate of TFP growth TFPG ${ }^{\mathrm{t}^{*}}$ was $1.240 \%$ per year. ${ }^{21}$ These average rates of TFP growth are substantial but considerably smaller than the corresponding average rates of TFP growth for the Corporate Nonfinancial Sector (which were $1.688 \%$ and $1.693 \%$ per year). From Table 3, it can be seen that there were cost efficiencies $e^{t}$ below 1 for the 20 consecutive years $1974-1993^{22}$ and in addition, for the years 1995, 2007, 2009 and 2010. Some of the cost efficiency declines were substantial; e.g., for 1983 , $\mathrm{e}^{1983}$ was equal to 0.8232 , indicating that optimal input cost was only $82.32 \%$ of observed input cost for that year. Thus the behavior and technology of the Noncorporate Nonfinancial Sector is very different from the behavior and technology of the Corporate Financial Sector. ${ }^{23}$

Again recall that equations (17)-(19) in section 2 above converted the growth decomposition for TFP into the levels decomposition $\mathrm{TFP}^{\mathrm{t}} \equiv \mathrm{y}^{\mathrm{t}} / \mathrm{X}^{\mathrm{t}}=\mathrm{E}^{\mathrm{t}} \mathrm{T}^{\mathrm{t}}$ defined by (20). Again define $\mathrm{X}^{\mathrm{t}^{*}}$ as the Fisher chained index level of input for year $t$ and again define the Fisher index level of TFP in year $t$ as $\mathrm{TFP}^{\mathrm{t}^{*}} \equiv \mathrm{y}^{\mathrm{t}} / \mathrm{X}^{\mathrm{t}^{*}}$. The Fisher input and productivity level series for the Noncorporate Nonfinancial Sector, $\mathrm{X}^{\mathrm{t}^{*}}$ and $\mathrm{TFP}^{\mathrm{t}^{*}}$, are also listed in Table 4 for comparison purposes. It can be seen that these indexes are very close to their nonparametric counterparts, $\mathrm{X}^{\mathrm{t}}$ and TFP $\mathrm{t}^{\mathrm{t}}$. However, it is no longer the case that the technology level series, $\mathrm{T}^{\mathrm{t}}$, is always close to $\mathrm{TFP}^{\mathrm{t}}$ and $\mathrm{TFP}^{\mathrm{t}^{*}}$ : when $\mathrm{e}^{\mathrm{t}}$ is substantially less than $1, \mathrm{TFP}^{\mathrm{t}}$ and $\mathrm{TFP}^{\mathrm{t}^{*}}$ are substantially below $\mathrm{T}^{\mathrm{t}}$. However, we note that at the end of the sample period, $\mathrm{TFP}^{\mathrm{t}^{*}}, \mathrm{TFP}^{\mathrm{t}}$ and $\mathrm{T}^{\mathrm{t}}$ end up at $1.9150,1.9456$ and 1.9456 respectively. Note that $\mathrm{y}^{\mathrm{t}}, \mathrm{X}^{\mathrm{t}}$ and $\mathrm{X}^{\mathrm{t}^{*}}$ are measured in billions of constant 1960 dollars.

Table 4: Noncorporate Nonfinancial Fisher TFP Levels TFP ${ }^{t^{*}}$, Nonparametric TFP Levels TFP ${ }^{t}$ Cumulated Cost Efficiency Levels $\mathbf{E}^{\mathrm{t}}$, Technology Levels $\mathbf{T}^{\mathrm{t}}$, Real Value Added $\mathbf{y}^{\mathbf{t}}$, Nonparametric Aggregate Input $X^{t}$ and Fisher Year $t$ Input Levels $X^{t^{*}}$

\begin{tabular}{cccccccc}
\hline Year & TFP $^{t^{*}}$ & TFP $^{\mathrm{t}}$ & $\mathbf{E}^{\mathrm{t}}$ & $\mathrm{T}^{\mathrm{t}}$ & $\mathbf{y}^{\mathrm{t}}$ & $\mathbf{X}^{\mathrm{t}}$ & $\mathbf{X}^{\mathrm{t}^{*}}$ \\
\hline $\mathbf{1 9 6 0}$ & $\mathbf{1 . 0 0 0 0}$ & $\mathbf{1 . 0 0 0 0}$ & $\mathbf{1 . 0 0 0 0}$ & $\mathbf{1 . 0 0 0 0}$ & $\mathbf{1 0 7 . 4 0}$ & $\mathbf{1 0 7 . 4 0}$ & $\mathbf{1 0 7 . 4 0}$ \\
$\mathbf{1 9 6 1}$ & $\mathbf{1 . 0 2 7 0}$ & $\mathbf{1 . 0 2 7 0}$ & $\mathbf{1 . 0 0 0 0}$ & $\mathbf{1 . 0 2 7 0}$ & $\mathbf{1 0 8 . 4 6}$ & $\mathbf{1 0 5 . 6 1}$ & $\mathbf{1 0 5 . 6 1}$ \\
$\mathbf{1 9 6 2}$ & $\mathbf{1 . 0 5 9 3}$ & $\mathbf{1 . 0 5 9 3}$ & $\mathbf{1 . 0 0 0 0}$ & $\mathbf{1 . 0 5 9 3}$ & $\mathbf{1 1 0 . 8 1}$ & $\mathbf{1 0 4 . 6 1}$ & $\mathbf{1 0 4 . 6 1}$ \\
$\mathbf{1 9 6 3}$ & $\mathbf{1 . 0 8 5 0}$ & $\mathbf{1 . 0 8 5 0}$ & $\mathbf{1 . 0 0 0 0}$ & $\mathbf{1 . 0 8 5 0}$ & $\mathbf{1 1 2 . 3 4}$ & $\mathbf{1 0 3 . 5 4}$ & $\mathbf{1 0 3 . 5 4}$ \\
$\mathbf{1 9 6 4}$ & $\mathbf{1 . 1 1 9 4}$ & $\mathbf{1 . 1 1 9 4}$ & $\mathbf{1 . 0 0 0 0}$ & $\mathbf{1 . 1 1 9 4}$ & $\mathbf{1 1 6 . 0 5}$ & $\mathbf{1 0 3 . 6 7}$ & $\mathbf{1 0 3 . 6 7}$ \\
$\mathbf{1 9 6 5}$ & $\mathbf{1 . 1 6 2 9}$ & $\mathbf{1 . 1 6 2 9}$ & $\mathbf{1 . 0 0 0 0}$ & $\mathbf{1 . 1 6 2 9}$ & $\mathbf{1 2 0 . 2 6}$ & $\mathbf{1 0 3 . 4 1}$ & $\mathbf{1 0 3 . 4 1}$ \\
$\mathbf{1 9 6 6}$ & $\mathbf{1 . 2 0 1 5}$ & $\mathbf{1 . 2 0 1 5}$ & $\mathbf{1 . 0 0 0 0}$ & $\mathbf{1 . 2 0 1 5}$ & $\mathbf{1 2 4 . 3 1}$ & $\mathbf{1 0 3 . 4 6}$ & $\mathbf{1 0 3 . 4 6}$ \\
$\mathbf{1 9 6 7}$ & $\mathbf{1 . 2 0 1 3}$ & $\mathbf{1 . 2 0 1 6}$ & $\mathbf{1 . 0 0 0 0}$ & $\mathbf{1 . 2 0 1 6}$ & $\mathbf{1 2 3 . 6 3}$ & $\mathbf{1 0 2 . 8 9}$ & $\mathbf{1 0 2 . 9 2}$ \\
1968 & $\mathbf{1 . 2 2 0 7}$ & $\mathbf{1 . 2 2 1 0}$ & $\mathbf{1 . 0 0 0 0}$ & $\mathbf{1 . 2 2 1 0}$ & $\mathbf{1 2 4 . 6 8}$ & $\mathbf{1 0 2 . 1 1}$ & $\mathbf{1 0 2 . 1 4}$ \\
\hline
\end{tabular}

\footnotetext{
${ }^{21}$ The corresponding geometric average rates of growth for our nonparametric rate of input growth $\gamma^{\mathrm{t}}$ was $1.137 \%$ per year while the corresponding geometric average Fisher index rate of input growth $\gamma^{t^{*}}$ was $1.166 \%$ per year.

${ }_{22}$ Evidently it took the Noncorporate Sector some 20 years to fully recover from the effects of the first oil shock recession in 1973-74. Another possibility is that there is a considerable amount of measurement error in our data for this sector.

${ }^{23}$ The Noncorporate Sector uses land and structure services more intensively while the Corporate Sector uses machinery and equipment services more intensively. Thus during recessions, it is much more difficult for the Noncorporate Sector to reduce its input usage due to the fixity of its land and structure inputs.
} 


\begin{tabular}{|c|c|c|c|c|c|c|c|}
\hline 1969 & 1.2248 & 1.2251 & 1.0000 & 1.2250 & 125.83 & 102.71 & 102.74 \\
\hline 1970 & 1.2245 & 1.2253 & 1.0000 & 1.2253 & 125.38 & 102.32 & 102.39 \\
\hline 1971 & 1.2433 & 1.2441 & 1.0000 & 1.2441 & 126.85 & 101.96 & 102.03 \\
\hline 1972 & 1.2926 & 1.2934 & 1.0000 & 1.2934 & 133.69 & 103.36 & 103.43 \\
\hline 1973 & 1.3982 & 1.3991 & 1.0000 & 1.3991 & 149.72 & 107.01 & 107.08 \\
\hline 1974 & 1.3143 & 1.3158 & 0.9404 & 1.3991 & 143.99 & 109.43 & 109.55 \\
\hline 1975 & 1.2854 & 1.2870 & 0.9199 & 1.3991 & 139.86 & 108.67 & 108.81 \\
\hline 1976 & 1.2844 & 1.2857 & 0.9189 & 1.3991 & 140.71 & 109.45 & 109.55 \\
\hline 1977 & 1.2743 & 1.2752 & 0.9114 & 1.3991 & 141.63 & 111.06 & 111.14 \\
\hline 1978 & 1.3122 & 1.3123 & 0.9380 & 1.3991 & 149.77 & 114.12 & 114.13 \\
\hline 1979 & 1.2731 & 1.2734 & 0.9101 & 1.3991 & 149.54 & 117.44 & 117.46 \\
\hline 1980 & 1.2357 & 1.2369 & 0.8841 & 1.3991 & 147.36 & 119.13 & 119.25 \\
\hline 1981 & 1.2207 & 1.2224 & 0.8737 & 1.3991 & 146.33 & 119.70 & 119.87 \\
\hline 1982 & 1.1860 & 1.1901 & 0.8506 & 1.3991 & 143.46 & 120.55 & 120.96 \\
\hline 1983 & 1.1458 & 1.1518 & 0.8232 & 1.3991 & 141.09 & 122.50 & 123.13 \\
\hline 1984 & 1.2788 & 1.2835 & 0.9174 & 1.3991 & 161.22 & 125.61 & 126.07 \\
\hline 1985 & 1.3022 & 1.3067 & 0.9340 & 1.3991 & 165.94 & 126.99 & 127.44 \\
\hline 1986 & 1.3387 & 1.3470 & 0.9627 & 1.3991 & 172.81 & 128.29 & 129.09 \\
\hline 1987 & 1.3202 & 1.3279 & 0.9491 & 1.3991 & 172.81 & 130.14 & 130.90 \\
\hline 1988 & 1.3509 & 1.3587 & 0.9711 & 1.3991 & 179.53 & 132.14 & 132.90 \\
\hline 1989 & 1.3367 & 1.3469 & 0.9627 & 1.3991 & 181.42 & 134.69 & 135.72 \\
\hline 1990 & 1.3239 & 1.3373 & 0.9558 & 1.3991 & 181.67 & 135.85 & 137.23 \\
\hline 1991 & 1.2770 & 1.2952 & 0.9257 & 1.3991 & 176.04 & 135.92 & 137.86 \\
\hline 1992 & 1.3599 & 1.3803 & 0.9865 & 1.3991 & 184.98 & 134.01 & 136.02 \\
\hline 1993 & 1.3482 & 1.3691 & 0.9785 & 1.3991 & 187.72 & 137.11 & 139.24 \\
\hline 1994 & 1.3861 & 1.4084 & 1.0000 & 1.4084 & 195.38 & 138.72 & 140.96 \\
\hline 1995 & 1.3673 & 1.3894 & 0.9865 & 1.4084 & 194.55 & 140.02 & 142.28 \\
\hline 1996 & 1.3898 & 1.4123 & 1.0000 & 1.4123 & 199.49 & 141.25 & 143.54 \\
\hline 1997 & 1.4025 & 1.4252 & 1.0000 & 1.4252 & 205.21 & 143.99 & 146.31 \\
\hline 1998 & 1.4704 & 1.4941 & 1.0000 & 1.4941 & 217.13 & 145.32 & 147.67 \\
\hline 1999 & 1.5280 & 1.5527 & 1.0000 & 1.5527 & 227.58 & 146.57 & 148.94 \\
\hline 2000 & 1.5435 & 1.5684 & 1.0000 & 1.5684 & 234.13 & 149.28 & 151.69 \\
\hline 2001 & 1.5757 & 1.6012 & 1.0000 & 1.6012 & 258.36 & 161.36 & 163.97 \\
\hline 2002 & 1.6059 & 1.6319 & 1.0000 & 1.6319 & 268.19 & 164.35 & 167.00 \\
\hline 2003 & 1.6314 & 1.6578 & 1.0000 & 1.6578 & 280.52 & 169.22 & 171.95 \\
\hline 2004 & 1.6801 & 1.7073 & 1.0000 & 1.7073 & 298.44 & 174.81 & 177.63 \\
\hline 2005 & 1.7034 & 1.7309 & 1.0000 & 1.7309 & 312.47 & 180.52 & 183.44 \\
\hline 2006 & 1.7684 & 1.7970 & 1.0000 & 1.7970 & 336.97 & 187.52 & 190.55 \\
\hline 2007 & 1.7221 & 1.7496 & 0.9737 & 1.7970 & 337.97 & 193.16 & 196.26 \\
\hline 2008 & 1.7984 & 1.8273 & 1.0000 & 1.8273 & 353.53 & 193.47 & 196.58 \\
\hline 2009 & 1.7512 & 1.7812 & 0.9748 & 1.8273 & 338.15 & 189.84 & 193.09 \\
\hline 2010 & 1.7574 & 1.7869 & 0.9779 & 1.8273 & 337.58 & 188.92 & 192.09 \\
\hline 2011 & 1.8653 & 1.8952 & 1.0000 & 1.8952 & 358.99 & 189.42 & 192.46 \\
\hline 2012 & 1.9035 & 1.9340 & 1.0000 & 1.9340 & 371.99 & 192.34 & 195.42 \\
\hline 2013 & 1.9085 & 1.9391 & 1.0000 & 1.9390 & 376.96 & 194.40 & 197.52 \\
\hline 2014 & 1.9150 & 1.9456 & 1.0000 & 1.9456 & 384.68 & 197.71 & 200.88 \\
\hline
\end{tabular}

The nonparametric TFP levels TFP ${ }^{t}$, cumulated cost efficiency factors $E^{t}$ (equal to the year $t$ cost efficiency factor $\mathrm{e}^{\mathrm{t}}$ ) and the year $\mathrm{t}$ level of technology $\mathrm{T}^{\mathrm{t}}$ for the Noncorporate Sector are graphed in Figure 2. It can be seen that except for recession years $t$ when $\mathrm{e}^{t}$ is less than 1 , the year $\mathrm{t}$ nonparametric TFP level $\mathrm{TFP}^{\mathrm{t}}$ is equal to the corresponding level of technology index $\mathrm{T}^{\mathrm{t}}$. During the years $\mathrm{t}$ when $\mathrm{e}^{\mathrm{t}}$ is less than 1 , $\mathrm{TFP}^{\mathrm{t}}$ falls below the technology index, $\mathrm{T}^{\mathrm{t}}$, since $\mathrm{TFP}^{\mathrm{t}} / \mathrm{T}^{\mathrm{t}}$ is equal to $\mathrm{e}^{\mathrm{t}}$.

Note that the level of technology index $\mathrm{T}^{\mathrm{t}}$ is constant for the years 1973-1993 and there is another flat spot on the plot for $\mathrm{T}^{\mathrm{t}}$ for the years 2008-2010. Finally, we note that there were two periods of rapid technological progress for this sector: the years 1960-1973 and the years 1993-2008. 


\section{Figure 2: Noncorporate Nonfinancial Sector TFP Levels TFP ${ }^{\mathrm{t}}$, Cumulated Cost Efficiency Factors $E^{t}$ and Levels of Technology $T^{t}$}

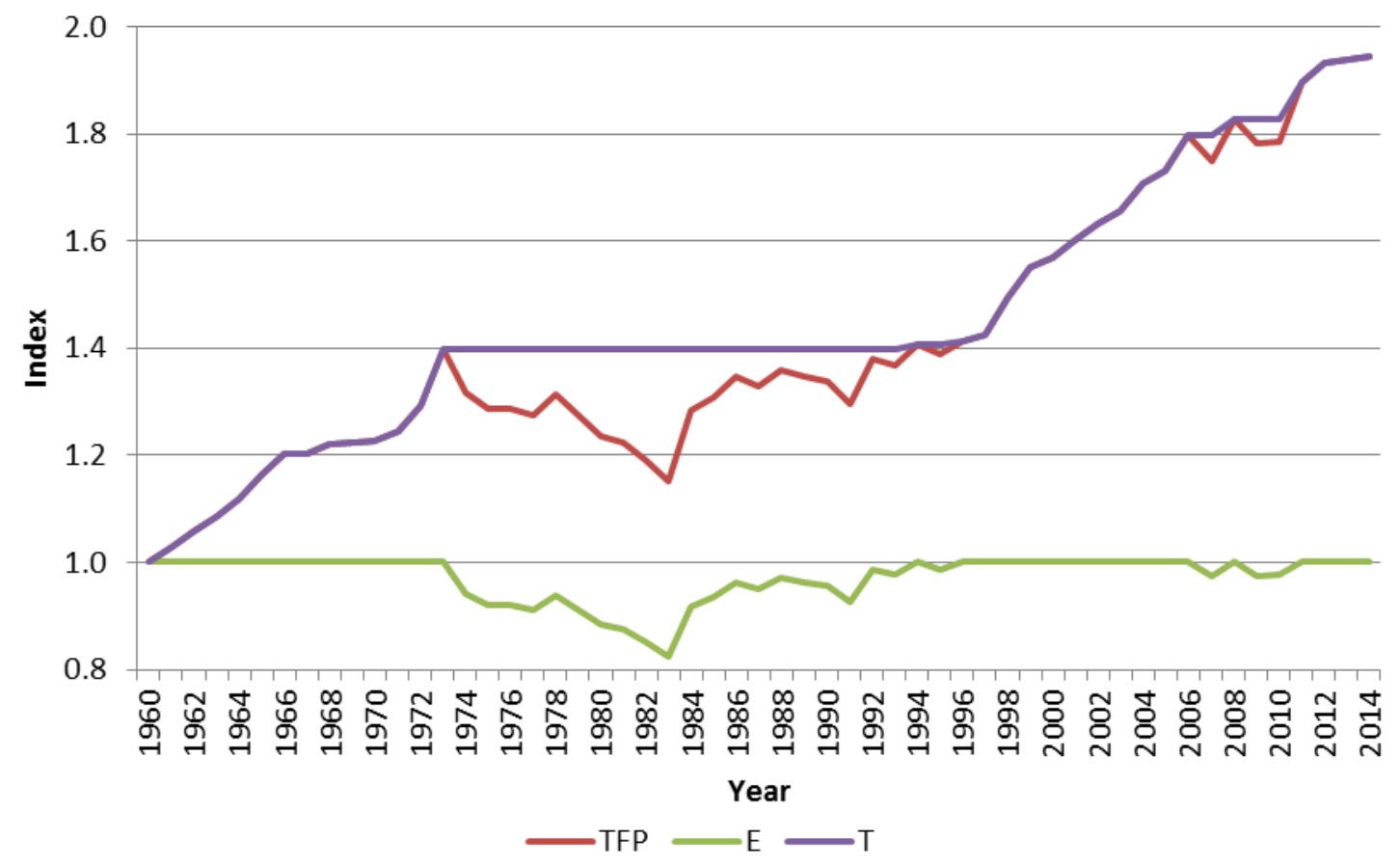

\section{Conclusion}

We have provided a fairly simple decomposition of TFP growth into technical progress and technical efficiency components. These components are relatively simple to compute and could be used by statistical agencies to provide more insight into the sources of Total Factor Productivity Growth. In particular, the efficiency component, when less than one shows the cost to the economy of recessions and underlines the importance of achieving macroeconomic stability.

For the U.S. Corporate Nonfinancial Sector there were cost inefficiencies in the following eleven years: 1969, 1970, 1974, 1979, 1980, 1982, 1989, 2001, 2007, 2008 and 2009. For 2009 there was a 3.28\% efficiency decline, providing a numerical estimate of the cost of the Great Recession.

The results for the Noncorporate Nonfinancial Sector were quite different, with cost inefficiencies for twenty consecutive years 1974-1993, and also for the years 1995, 2007, 2009 and 2010. For 1983, cost inefficiency was equal to 0.8232 , meaning that optimal input cost was only $82.32 \%$ of observed input cost for that year. Hence the behavior and technology of the Noncorporate Nonfinancial Sector is very different from the behavior and technology of the Corporate Financial Sector. ${ }^{24}$ Such differences between major sectors of the economy are informative as to the sources of productivity slowdowns and possible policy responses.

\footnotetext{
${ }^{24}$ The finding that cost inefficiency is so much bigger in the Noncorporate Sector can be at least partially explained by the fact that this sector uses land and structures much more intensively than the Corporate Sector, which uses machinery and equipment more intensively. Thus when there is a recession, due to the quasi-fixed nature of the land and structure inputs, the Noncorporate Sector cannot reduce their use of these inputs.
} 
We have also shown that standard Fisher index measure of TFP growth will provide an adequate long run measure of growth in TFP. Thus our methodology should be viewed as a complement to standard statistical agency measures of TFP growth. Our decomposition is different from standard growth accounting decomposition approaches and gives policy makers information on two important components of TFP growth, while avoiding the interpretation of productivity declines implying disappearing technologies.

In Appendix B, we show how our one output methodology can be extended to the case of many outputs. However, it should be noted that the multiple output joint cost function $\mathrm{C}(\mathrm{y}, \mathrm{w}, \mathrm{t})$ defined by equation (B1) in Appendix B may not be well defined; i.e., the linear program defined in (B1) may not have a feasible solution if the output vector $y$ has some negative components in it that represent amounts used of intermediate inputs. Even if all components of the $\mathrm{y}$ vector are positive, the optimal costs defined by $\mathrm{C}(\mathrm{y}, \mathrm{w}, \mathrm{t})$ can be quite volatile and this will tend to make the methodology based on this nonparametric cost function impractical for statistical agencies to use. Thus the one output methodology that we have illustrated above may be the most suitable application for statistical agencies that provide TFP measures.

\section{Appendix A: U.S. Corporate and Noncorporate Nonfinancial Sector Data}

The output and input price data that we use in this paper are drawn from Diewert and Fox (2016b). These data were constructed from Bureau of Economic Analysis (BEA) National Income and Product Accounts Tables, BEA Capital Stock Tables, the Integrated Macroeconomic Accounts Tables constructed by the BEA, Bureau of Labor Statistics and the Board of Governors of the Federal Reserve and published by the BEA. Diewert and Fox also used other information on the price and quantity of farm land and on commercial property in the U.S. Diewert and Fox used the data from these sources to construct productivity accounts for two subsectors of the U.S. private sector: the Corporate Nonfinancial Sector and the Noncorporate Nonfinancial Sector. For convenience, we list the data used for these two subsectors in the present study in this Appendix. ${ }^{25}$ The user costs for the various capital stocks that we list here are the user costs that use predicted end of period asset prices rather than actual end of period asset prices in the user cost formula. Our use of predicted prices dramatically smoothed the resulting user costs and in particular, eliminated the negative user costs that occurred when actual end of period asset prices were used in the user cost formula. We used balancing rates of return in our user costs so that the value of inputs was equal to the corresponding value added for each year and for each of our two sectors.

The output and input price information for the U.S. Corporate Nonfinancial Sector are listed in Table A1. The first input is labour input and the remaining input prices are user $\operatorname{costs}^{26}$ for

\footnotetext{
${ }^{25}$ Diewert and Fox (2016b) did not construct data for the third subsector of the U.S. Private Sector economy which is the Financial Sector. There is no general consensus on how to measure outputs and inputs for the Financial Sector.

${ }^{26}$ Diewert and Fox used end of period user costs as the price for the services of a unit of capital. The period $\mathrm{t}$ user cost $\mathrm{u}^{\mathrm{t}}$ for an asset with beginning of year $\mathrm{t}$ asset price $\mathrm{P}^{\mathrm{t}}$ is defined as $\mathrm{u}^{\mathrm{t}} \equiv\left[1+\mathrm{r}^{\mathrm{t}}-\left(1+\mathrm{i}^{\mathrm{t}}\right)(1-\delta)\right] \mathrm{P}^{\mathrm{t}}=\left[\mathrm{r}^{\mathrm{t}}-\mathrm{i}^{\mathrm{t}}+\right.$ $\left.\left(1+\mathrm{i}^{\mathrm{t}}\right) \delta\right] \mathrm{P}^{\mathrm{t}}$ where $\delta$ is the geometric depreciation rate, $\mathrm{r}^{\mathrm{t}}$ is the year $\mathrm{t}$ cost of capital and $\mathrm{i}^{\mathrm{t}}$ is either the ex post asset inflation rate for year $\mathrm{t}$ or an anticipated asset inflation rate. This formula for the user cost of capital was obtained by Christensen and Jorgenson $(1969 ; 302)$ using ex post asset inflation rates. Diewert and Fox used anticipated asset inflation rates where the anticipated rate in year $t$ was essentially the geometric average growth rate of the asset price over the 25 years prior to year $t$.
} 
nine types of capital, where the stock price was set equal to unity in 1960 for each asset. The nine types of asset and their input number are as follows: $2=$ Equipment; $3=$ Intellectual property products; $4=$ Nonresidential structures; $5=$ Residential structures; $6=$ Residential land; $7=$ Farm land; $8=$ Commercial land; $9=$ Beginning of year inventory stocks and $10=$ Beginning of the year real holdings of currency and deposits. The quantity (or volume) data that match up with the price data in Table A1 are listed in Table A2.

The output and input price information for the U.S. Noncorporate Nonfinancial Sector are listed in Table A3. The first input is labour input and the remaining input prices are user costs for 14 types of capital, where the stock price was set equal to unity in 1960 for each asset. The 14 types of asset and their input number are as follows: $2=$ Equipment held by sole proprietors; 3 = Equipment held by partners; 4 = Equipment held by cooperatives; $5=$ Intellectual property products held by sole proprietors; $6=$ Intellectual property products held by partners; $7=$ Nonresidential structures held by sole proprietors; $8=$ Nonresidential structures held by partners; $9=$ Nonresidential structures held by cooperatives; ; $10=$ Residential structures held by the noncorporate nonfinancial sector; $11=$ Residential land held by the noncorporate nonfinancial sector; $12=$ Farm land held by the noncorporate nonfinancial sector; $13=$ Commercial land held by noncorporate nonfinancial sector; $14=$ Beginning of the year inventories held by the noncorporate nonfinancial sector and $15=$ Beginning of the year real holdings of currency and deposits by noncorporate nonfinancial sector.

The quantity (or volume) data that match up with the above price data for the Noncorporate Nonfinancial Sector are listed in Table A4.

Table A1: Value Added Output Price Indexes $p^{t}$ and Input Price Indexes $w_{1}{ }^{t}$ for Labour and $\mathrm{w}_{2}{ }^{\mathrm{t}}-\mathrm{w}_{10}{ }^{\mathrm{t}}$ for Capital Services for the U.S. Corporate Nonfinancial Sector, 1960-2014

\begin{tabular}{|c|c|c|c|c|c|c|c|c|c|c|c|}
\hline Year & $p^{t}$ & $w_{1}^{t}$ & $w_{2}{ }^{t}$ & $w_{3}{ }^{t}$ & $w_{4}{ }^{t}$ & $w_{5}{ }^{t}$ & $w_{6}{ }^{t}$ & $w_{7}{ }^{t}$ & $w_{8}{ }^{t}$ & $\mathrm{w}_{9}{ }^{t}$ & $W_{10}^{t}$ \\
\hline 1960 & 1.00000 & 1.00000 & 0.19256 & 0.26274 & 0.10410 & .09655 & $\begin{array}{l}0.07678 \\
\end{array}$ & .02889 & 0.08036 & .08052 & 0.06627 \\
\hline 1961 & 305 & 00 & 121 & 13 & & 68 & 97987 & 63 & 7546 & & \\
\hline 1962 & 1.00954 & & 0442 & 8147 & 475 & 1000 & 09169 & 4384 & 08626 & 99257 & 07969 \\
\hline 1963 & 1.01454 & 1.10939 & 0.21268 & 0.29413 & 0.12139 & 111579 & 0.10456 & 05301 & 0.09376 & 10000 & 08741 \\
\hline 1964 & 1.02388 & 1.16396 & 0.22183 & 0.30549 & 0.12856 & 0.12127 & 0.11296 & 0.06475 & 0.10190 & 0.10479 & 0.09581 \\
\hline 1965 & 1.04188 & 1.20847 & 0.23669 & 0.32458 & 0.14241 & 0.13640 & 0.11341 & 0.08218 & 0.11494 & 0.11501 & 0.10830 \\
\hline 1966 & 1.07230 & 1.27923 & 0.24516 & $\mathbf{0 . 3 3 8 9 7}$ & 0.14790 & 0.14206 & 0.12100 & 0.08799 & 0.11795 & 0.11812 & 0.11505 \\
\hline 1967 & 1. & & & 53 & 29 & 446 & 1277 & 943 & 874 & 27 & 0712 \\
\hline 1968 & 024 & 89 & 11 & 250 & 20 & 841 & 292 & 31 & 185 & 292 & 1043 \\
\hline 1969 & 785 & 45 & 606 & 80 & 58 & 30 & 0370 & 991 & 519 & 651 & 403 \\
\hline 1970 & 1.22161 & 1.66899 & 0.23706 & 0.35763 & 0.12647 & 0.11951 & 0.07744 & 0.06326 & 0.08785 & .09230 & 08892 \\
\hline 1971 & 1.26574 & 1.77389 & 0.25229 & 0.38147 & 0.13929 & 0.13209 & 0.08240 & 0.08156 & 0.09820 & 0.10138 & 0.09973 \\
\hline 1972 & 1.3 & & & 67 & & & 0.09972 & & 0.11083 & 142 & 11193 \\
\hline 1973 & & & & & & & & 866 & & & 2109 \\
\hline & & & & & & & 067 & 29 & & 298 & 1295 \\
\hline 197 & 52 & & 942 & 289 & & 105 & 0.08722 & 12 & 316 & 104 & 2395 \\
\hline 1976 & 491 & 2.62329 & 0.36716 & 4321 & 566 & 19687 & 0.10911 & 12218 & 0.14793 & .14343 & 0.14440 \\
\hline 1977 & 1.85432 & 3198 & 0413 & 0.58130 & 0.24237 & 1878 & 0.13752 & 4120 & 0.16947 & 15795 & 0.16026 \\
\hline 1978 & 1.98679 & 3.07219 & 0.43817 & 0.61872 & 0.26551 & 0.24329 & 0.14831 & 0.16334 & 0.18682 & 0.16864 & 0.17269 \\
\hline 1979 & 2.14974 & 3.36681 & & 0.65391 & 37 & 4602 & 0.10610 & 3216 & 8151 & .16846 & 0.16952 \\
\hline 1980 & & & & & & 4192 & & & & 16147 & 16096 \\
\hline & & & & & & & & & & & 18884 \\
\hline & & & & & & 92 & & & & & 7749 \\
\hline 1093 & 5464 & 65 & 07 & 50 & 86 & 378 & 36 & 12 & 494 & 362 & 0.19835 \\
\hline 0 & 3966 & 4.78376 & 470 & 0142 & 08 & 5503 & 0.28351 & 0.28564 & 0.27945 & .23090 & 0.23849 \\
\hline 1985 & 2.89001 & 5.04887 & 0.62004 & 1881 & 62 & 0.35491 & 0.27704 & 0.28537 & 0.27793 & .22652 & 0.23644 \\
\hline 1986 & 2.93035 & 5.32469 & 0.60603 & 0.91172 & $\mathbf{0 . 3 6 3 3 7}$ & 0.34405 & 0.34997 & 0.32864 & 0.26629 & 0.19382 & 0.22287 \\
\hline 1987 & 2.9 & 260 & 0.63339 & 4070 & 0.3 & 8132 & 0.40407 & 4765 & 0.30274 & 0.2 & 23946 \\
\hline & 3.06238 & 5.82700 & 0.66869 & 0.99297 & & 0.42055 & 0.4 & 52 & 0.35243 & 0.23015 & 0.26237 \\
\hline 1989 & 3.15539 & 6.01745 & 0.67102 & 1.01225 & 0.41931 & 0.41277 & 0.50524 & 0.42883 & 0.34280 & 0.23860 & 0.25451 \\
\hline
\end{tabular}




\begin{tabular}{|c|c|c|c|c|c|c|c|c|c|c|c|}
\hline 1990 & 3.25074 & 6.33621 & 0.67795 & 1.02431 & 0.42395 & 0.40154 & 0.53776 & 0.41802 & 0.32516 & 0.22965 & 0.24551 \\
\hline 1991 & 3.32219 & 6.65680 & 0.68223 & 1.04054 & 0.41384 & 0.38647 & 0.57116 & 0.42496 & 0.31992 & 0.21556 & 0.23699 \\
\hline 1992 & 3.36449 & 6.99763 & 0.68436 & 1.03788 & 0.40575 & 0.38768 & 0.58334 & 0.44836 & 0.32374 & 0.20533 & 0.23064 \\
\hline 1993 & 3.43456 & 7.10638 & 0.70682 & 1.06689 & 0.43069 & 0.42736 & 0.71268 & 0.48853 & 0.35962 & 0.21578 & 0.24861 \\
\hline 1994 & 3.48679 & 7.25300 & 0.75313 & 1.11924 & 0.48826 & 0.47959 & 0.93686 & 0.59185 & 0.43389 & 0.25403 & 0.29332 \\
\hline 1995 & 3.53437 & 7.39298 & 0.78121 & 1.17658 & 0.51979 & 0.50898 & 0.98009 & 0.64555 & 0.46164 & 0.27131 & 0.31057 \\
\hline 1996 & 3.55779 & 7.68905 & $\mathbf{0 . 8 0 3 8 3}$ & 1.21993 & 0.55075 & 0.53839 & 0.98237 & 0.70123 & 0.50483 & 0.29167 & .33388 \\
\hline 1997 & 3.58599 & 7.96034 & 0.81598 & 1.27200 & 0.58614 & 0.57325 & 1.00470 & 0.72237 & 0.54560 & 0.29511 & 0.35232 \\
\hline 1998 & 3.59545 & 8.48754 & 0.78691 & 1.27544 & 0.57043 & 0.55798 & 0.90282 & 0.67356 & $\mathbf{0 . 5 2 7 5 3}$ & 0.26988 & 0.32898 \\
\hline 1999 & 3.61926 & 8.85048 & 0.77469 & 1.31806 & 0.56243 & 0.56739 & 0.94087 & 0.66969 & 0.54253 & 0.26082 & 0.32448 \\
\hline 2000 & 3.66103 & 9.45242 & 0.75184 & 1.35132 & $\mathbf{0 . 5 3 7 1 3}$ & 0.55208 & 0.87348 & 0.57220 & 0.51378 & 0.24160 & 0.29908 \\
\hline 2001 & 3.71320 & 9.82805 & 0.70784 & 1.31973 & 0.49969 & 0.51276 & 0.76280 & 0.43952 & 0.44344 & 0.20973 & 0.26160 \\
\hline 2002 & 3.71089 & 10.03735 & 0.71070 & 1.31519 & $\mathbf{0 . 5 3 8 4 3}$ & 0.56070 & 0.97163 & 0.43830 & 0.50197 & 0.21640 & 0.28450 \\
\hline 2003 & 3.74861 & 10.40118 & 0.72529 & 1.34037 & 0.59229 & 0.62017 & 1.21573 & 0.51345 & 0.59073 & 0.24184 & 0.32026 \\
\hline 2004 & 3.82615 & 10.82730 & 0.75909 & 1.38485 & 0.67653 & 0.70191 & 1.58749 & 0.61013 & 0.74340 & 0.27886 & 0.36908 \\
\hline 2005 & 3.95768 & 11.21466 & 0.80199 & 1.44142 & $\mathbf{0 . 7 8 9 3 7}$ & 0.78852 & 2.00121 & 0.73406 & 0.87517 & 0.31149 & 0.41586 \\
\hline 2006 & 4.07891 & 11.61647 & 0.83696 & 1.49294 & 0.90066 & 0.87282 & 2.24793 & 0.86854 & 0.98306 & 0.34048 & 0.45815 \\
\hline 2007 & 4.16098 & 12.07780 & 0.83088 & 1.49253 & 0.88964 & 0.84784 & 2.08966 & 0.82053 & 0.91385 & 0.32041 & 0.43718 \\
\hline 2008 & 4.24922 & 12.42257 & 0.81797 & 1.48885 & $\mathbf{0 . 8 7 5 3 5}$ & 0.81287 & 1.84853 & 0.75691 & 0.80840 & 0.32167 & 0.42098 \\
\hline 2009 & 4.31825 & 12.65447 & 0.78688 & 1.43364 & 0.81163 & 0.74772 & 1.56751 & 0.67751 & 0.68550 & 0.28203 & 0.39094 \\
\hline 2010 & 4.32164 & 12.88069 & 0.84979 & 1.51054 & 0.95496 & 0.87881 & 1.98741 & 0.95722 & 0.86913 & 0.34693 & 0.48488 \\
\hline 2011 & 4.41763 & 13.16690 & 0.87482 & 1.53668 & 1.00154 & 0.90534 & 1.91087 & 1.04686 & 0.83566 & 0.37164 & 0.50267 \\
\hline 2012 & 4.49159 & 13.48156 & 0.90515 & 1.57710 & 1.08104 & 0.95401 & 1.96570 & 1.19245 & 0.85964 & 0.40289 & 0.53983 \\
\hline 2013 & 4.52053 & 13.62476 & 0.90913 & 1.59736 & 1.12044 & 0.99709 & 2.20110 & 1.30349 & 0.96258 & 0.40288 & 0.54803 \\
\hline 2014 & 4.55684 & 13.95482 & 0.89901 & 1.60571 & 1.11386 & 1.00166 & 2.33484 & 1.27996 & 1.02107 & 0.37644 & 0.52966 \\
\hline
\end{tabular}

Table A2: Real Value Added Output Volumes $y^{t}$ and Input Quantities $x_{1}{ }^{t}$ for Labour and $\mathbf{x}_{2}{ }^{t}-\mathbf{x}_{10}{ }^{t}$ for Capital Services for the U.S. Corporate Nonfinancial Sector, 1960-2014 in Billions of 1960 U.S. Dollars

\begin{tabular}{|c|c|c|c|c|c|c|c|c|c|c|c|}
\hline Year & $y^{t}$ & $\mathrm{x}_{1}{ }^{\mathrm{t}}$ & $\mathbf{x}_{2}{ }^{t}$ & $\mathbf{x}_{3}{ }^{t}$ & $\mathbf{x}_{4}{ }^{t}$ & $\mathbf{x}_{5}{ }^{t}$ & $\mathbf{x}_{6}{ }^{t}$ & $x_{7}{ }^{t}$ & $x_{8}{ }^{t}$ & $\mathrm{x}_{9}{ }^{\mathrm{t}}$ & $\mathbf{x}_{10}{ }^{t}$ \\
\hline 1960 & 255.90 & 180.40 & 136.30 & 23.30 & 256.90 & 4.70 & 1.17 & 13.43 & 79.21 & 87.40 & 31.00 \\
\hline 1961 & 262.00 & 179.30 & 141.30 & 24.91 & 262.41 & 5.19 & 1.27 & 13.41 & 80.43 & 89.86 & 31.13 \\
\hline 1962 & 284.19 & 185.83 & 145.36 & 26.69 & 267.71 & 5.71 & 1.38 & 13.40 & 81.58 & 91.29 & 35.43 \\
\hline 1963 & 301.71 & 189.38 & 151.17 & 28.41 & 273.13 & 6.34 & 1.53 & 13.38 & 82.74 & 95.51 & 39.21 \\
\hline 1964 & 322.89 & 193.91 & 157.80 & 30.43 & 278.37 & 6.92 & 1.63 & 13.33 & 83.82 & 102.30 & 38.24 \\
\hline 1965 & 350.04 & 203.07 & 166.42 & 32.35 & 285.12 & 7.56 & 1.78 & 13.26 & 85.34 & 108.93 & 38.28 \\
\hline 1966 & 375.92 & 213.33 & 179.03 & 34.84 & 294.21 & 8.02 & 1.89 & 13.14 & 87.53 & 114.65 & 39.93 \\
\hline 1967 & 386.69 & 215.62 & 195.16 & 37.96 & 304.82 & 8.72 & 2.05 & 12.99 & 90.14 & 127.78 & 37.80 \\
\hline 1968 & 411.77 & 221.02 & 208.63 & 41.17 & 315.05 & 9.23 & 2.17 & 12.82 & 92.60 & 138.18 & 40.09 \\
\hline 1969 & 428.24 & 229.08 & 222.80 & 44.43 & 325.33 & 9.41 & 2.22 & 12.62 & 95.03 & 144.62 & 40.71 \\
\hline 1970 & 424.52 & 224.39 & 238.11 & 47.56 & 336.33 & 9.72 & 2.34 & 12.41 & 97.64 & 151.24 & 38.71 \\
\hline 1971 & 441.24 & 223.35 & 250.13 & 49.82 & 347.09 & 9.98 & 2.40 & 12.20 & 100.14 & 154.24 & 38.69 \\
\hline 1972 & 474.69 & 233.38 & 260.20 & 51.50 & 356.88 & 10.35 & 2.45 & 12.02 & 102.32 & 157.40 & 39.66 \\
\hline 1973 & 503.52 & 245.62 & 273.63 & 53.63 & 367.04 & 10.89 & 2.54 & 11.89 & 104.58 & 157.42 & 42.43 \\
\hline 1974 & 495.88 & 245.21 & 292.51 & 55.75 & 378.21 & 11.31 & 2.61 & 11.82 & 107.08 & 158.63 & 44.46 \\
\hline 1975 & 488.85 & 234.31 & 310.48 & 57.59 & 389.20 & 11.70 & 2.72 & 11.83 & 109.49 & 180.63 & 43.99 \\
\hline 1976 & 528.86 & 243.97 & 320.07 & 58.89 & 397.51 & 11.79 & 2.74 & 11.89 & 111.11 & 177.49 & 47.78 \\
\hline 1977 & 567.81 & 255.40 & 330.91 & 61.22 & 405.73 & 11.82 & 2.67 & 11.95 & 112.68 & 190.31 & $\mathbf{5 0 . 8 7}$ \\
\hline 1978 & 604.69 & 270.00 & 347.72 & 64.19 & 414.14 & 11.92 & 2.62 & 11.97 & 114.27 & 201.26 & 54.06 \\
\hline 1979 & 624.08 & 279.91 & 369.13 & 67.30 & 425.62 & 12.07 & 2.59 & 11.93 & 116.67 & 206.24 & 55.68 \\
\hline 1980 & 617.97 & 276.25 & 392.10 & 71.35 & 439.13 & 12.31 & 2.57 & 11.83 & 119.58 & 214.11 & 54.97 \\
\hline 1981 & 643.05 & 279.73 & 409.11 & 75.30 & 453.99 & 12.46 & 2.57 & 11.71 & 122.81 & 215.05 & 51.13 \\
\hline 1982 & 629.01 & 269.42 & 425.13 & 80.35 & 469.41 & 12.56 & 2.59 & 11.60 & 126.14 & 230.39 & 44.56 \\
\hline 1983 & 659.80 & 273.81 & 433.71 & 85.53 & 482.96 & 12.57 & 2.60 & 11.52 & 128.91 & 224.53 & 48.06 \\
\hline 1984 & 718.57 & 290.19 & 441.74 & 91.16 & 492.95 & 12.68 & 2.59 & 11.46 & 130.69 & 223.74 & 56.80 \\
\hline 1985 & 751.87 & 295.14 & 458.19 & 98.48 & 506.51 & 12.84 & 2.58 & 11.41 & 133.37 & 245.05 & 58.41 \\
\hline 1986 & 771.48 & 296.39 & 473.93 & 106.18 & 521.46 & 13.03 & 2.57 & 11.36 & 136.37 & 253.96 & 64.35 \\
\hline 1987 & 812.39 & 304.65 & 485.39 & 113.59 & 531.66 & 13.27 & 2.56 & 11.30 & 138.08 & 259.07 & 69.51 \\
\hline 1988 & 862.30 & 313.25 & 493.06 & 119.86 & 541.10 & 13.45 & 2.53 & 11.23 & 139.56 & 266.81 & 69.04 \\
\hline 1989 & 878.69 & 321.53 & 502.49 & 126.00 & 549.97 & 13.56 & 2.51 & 11.15 & 140.86 & 270.62 & 70.58 \\
\hline 1990 & 891.40 & 321.56 & 513.17 & 133.47 & 558.87 & 13.68 & 2.49 & 11.07 & 142.13 & 279.81 & 68.10 \\
\hline 1991 & 886.79 & 311.13 & 523.24 & 141.59 & 571.46 & 13.75 & 2.48 & 11.00 & 144.30 & 284.15 & 64.92 \\
\hline 1992 & 913.84 & 312.78 & 529.82 & 149.42 & 580.43 & 13.86 & 2.48 & 10.98 & 145.52 & 285.39 & 66.24 \\
\hline 1993 & 936.36 & 319.57 & 539.56 & 156.86 & 587.08 & 13.94 & 2.45 & 10.99 & 146.13 & 288.02 & 62.99 \\
\hline 1994 & 993.98 & 330.72 & 555.99 & 164.36 & 594.31 & 14.02 & 2.42 & 11.03 & 146.86 & 294.53 & 68.76 \\
\hline 1995 & 1041.97 & 341.49 & 578.75 & 171.62 & 600.71 & 14.18 & 2.41 & 11.08 & 147.36 & 312.83 & 71.42 \\
\hline
\end{tabular}




\begin{tabular}{|c|c|c|c|c|c|c|c|c|c|c|c|}
\hline 1996 & 1103.04 & 346.95 & 608.03 & 180.65 & 610.50 & 14.43 & 2.42 & 11.12 & 148.67 & 323.14 & 72.11 \\
\hline 1997 & 1176.66 & 359.61 & 640.06 & 192.35 & 621.99 & 14.65 & 2.42 & 11.14 & 150.34 & 331.08 & 76.01 \\
\hline 1998 & 1243.46 & 364.51 & 676.37 & 206.79 & 633.94 & 15.00 & 2.43 & 11.13 & 152.09 & 351.46 & 79.77 \\
\hline 1999 & 1311.12 & 373.99 & 714.79 & 220.93 & 647.08 & 15.32 & 2.42 & 11.09 & 154.08 & 373.25 & 83.47 \\
\hline 2000 & 1382.97 & 380.57 & 758.83 & 236.72 & 659.77 & 15.71 & 2.43 & 11.02 & 155.91 & 391.40 & 97.80 \\
\hline 2001 & 1353.60 & 364.73 & 807.14 & 253.06 & 675.31 & 16.17 & 2.46 & 10.94 & 158.37 & 406.54 & 108.50 \\
\hline 2002 & 1365.17 & 352.88 & 836.85 & 264.77 & 688.89 & 16.59 & 2.48 & 10.86 & 160.31 & 396.48 & 96.15 \\
\hline 2003 & 1394.84 & 345.70 & 849.80 & 270.94 & 695.87 & 17.04 & 2.47 & 10.78 & 160.69 & 400.70 & 94.92 \\
\hline 2004 & 1457.60 & 347.53 & 864.24 & 277.85 & 701.20 & 17.55 & 2.48 & 10.72 & 160.65 & 404.04 & 119.48 \\
\hline 2005 & 1505.65 & 350.46 & 883.71 & 285.80 & 704.80 & 18.07 & 2.51 & 10.65 & 160.21 & 422.06 & 126.94 \\
\hline 2006 & 1563.63 & 355.47 & 911.51 & 297.23 & 708.42 & 18.60 & 2.52 & 10.58 & 159.76 & 437.87 & 145.58 \\
\hline 2007 & 1579.29 & 356.46 & 948.76 & 309.84 & 716.01 & 19.03 & 2.54 & 10.50 & 160.18 & 456.20 & 127.52 \\
\hline 2008 & 1558.90 & 350.81 & 982.79 & 322.87 & 727.05 & 19.36 & 2.58 & 10.43 & 161.34 & 431.97 & 112.36 \\
\hline 2009 & 1448.25 & & 1003.81 & 335.42 & 739.66 & 19.56 & 2.61 & & & & 73.39 \\
\hline 2010 & 1528.52 & 322.86 & 989.83 & 342.65 & 745.03 & 19.58 & 2.62 & 10.36 & 162.65 & 422.76 & 116.40 \\
\hline 2011 & 1566.84 & 331.39 & 993.14 & 352.42 & 746.62 & 19.53 & 2.62 & 10.37 & 161.66 & 433.31 & 140.21 \\
\hline 2012 & 1630.05 & 340.71 & 1012.94 & 362.86 & 749.80 & 19.51 & 2.64 & 10.41 & 160.99 & 438.69 & 142.93 \\
\hline 2013 & 1679.43 & 348.44 & 1042.71 & 373.67 & 755.97 & 19.57 & 2.63 & 10.50 & 160.96 & 453.65 & 138.55 \\
\hline 2014 & 1732.74 & 358.00 & 1072.23 & 386.19 & 762.97 & 19.69 & 2.66 & 10.50 & 161.08 & 469.18 & 161.67 \\
\hline
\end{tabular}

Table A3: Value Added Output Price Indexes $p^{t}$ and Input Price Indexes $w_{1}{ }^{t}$ for Labour and $\mathrm{w}_{2}{ }^{\mathrm{t}}-\mathrm{w}_{15}{ }^{\mathrm{t}}$ for Capital Services for the U.S. Noncorporate Nonfinancial Sector, 1960-2014

\begin{tabular}{|c|c|c|c|c|c|c|c|c|}
\hline Year & $p^{t}$ & $w_{1}{ }^{t}$ & $w_{2}{ }^{t}$ & $w_{3}{ }^{t}$ & $w_{4}{ }^{t}$ & $w_{5}{ }^{t}$ & $w_{6}{ }^{t}$ & $\mathrm{w}_{7}{ }^{\mathrm{t}}$ \\
\hline 1960 & 1.00000 & 1.00000 & 22676 & 0.21874 & 0.18916 & 0.17713 & 20184 & 08767 \\
\hline 1961 & 01608 & 02966 & 54 & 22458 & 19258 & .22663 & .20632 & 09207 \\
\hline 1962 & 63 & 07195 & 24029 & 22913 & 01 & .22519 & 9617 & 09648 \\
\hline 19 & & & & 3621 & & & & 111 \\
\hline 1964 & & & & 4363 & & & & 461 \\
\hline 1965 & 1.08098 & 1.20531 & 0.26270 & 0.25396 & 64 & 166 & 25750 & 11968 \\
\hline 1966 & 1.11417 & 1.26753 & 0.27539 & 0.26764 & 0.20379 & 0.29442 & 0.26760 & 0.13079 \\
\hline 196 & 1.14936 & 1.3 & 0.27994 & 0.26851 & & & 47 & 12965 \\
\hline & & 21 & & 0.27683 & & & 725 & 666 \\
\hline 196 & & 32 & & 8596 & & & 114 & 373 \\
\hline 1970 & 323 & 164 & & 0.29090 & & & 899 & 3984 \\
\hline 1971 & & 181 & & 29890 & & & 7216 & 0.14877 \\
\hline 197 & & 55 & & 0.32034 & 04 & & 731 & 16980 \\
\hline & & & & & & & 990 & 811 \\
\hline 19 & & & & 718 & & & 493 & 257 \\
\hline 197 & & & & & & & & 935 \\
\hline 197 & & 11 & & & & & & 0.23953 \\
\hline 197 & & 36 & & 20 & & & 32 & 667 \\
\hline & & & & & & & 33 & 977 \\
\hline 1979 & & & & & & & 300 & 527 \\
\hline & & & & & & & & \\
\hline 198 & & & & & & & & \\
\hline & & 3 & & 01 & & & & 908 \\
\hline & & & & & & & & 300 \\
\hline 19 & & & & 65 & & & 34 & 341 \\
\hline & & & & & & & & 407 \\
\hline 1986 & & & & 0.71484 & & & & 796 \\
\hline & & & & & & & & \\
\hline & & & & & & & & 796 \\
\hline & & & & & & & 33 & 0148 \\
\hline & & & & 0.78002 & & & 1.14971 & 0.50378 \\
\hline & & 68 & & 0.76984 & & 370 & 6579 & 0.47152 \\
\hline & & & & & & & & 681 \\
\hline & & & & 21 & & & 670 & 0.50657 \\
\hline & & & & & & & 1.21956 & 0.52853 \\
\hline & & 6.77705 & & 5329 & & & 6365 & 0.55904 \\
\hline & & & & 25 & & 395 & 232 & 0.62418 \\
\hline & & & & & & & & 0.66305 \\
\hline 199 & 5.66193 & 7.79292 & 1.07972 & 0.94669 & 0.68875 & 1.42473 & 1.49293 & 0.72412 \\
\hline
\end{tabular}




\begin{tabular}{lllllllll}
\hline $\mathbf{1 9 9 9}$ & $\mathbf{5 . 7 6 6 3 5}$ & $\mathbf{8 . 1 3 4 6 7}$ & $\mathbf{1 . 0 9 3 7 4}$ & $\mathbf{0 . 9 6 4 6 8}$ & $\mathbf{0 . 6 8 7 3 4}$ & $\mathbf{1 . 5 1 7 8 8}$ & $\mathbf{1 . 6 3 9 7 2}$ & $\mathbf{0 . 7 6 2 6 5}$ \\
$\mathbf{2 0 0 0}$ & $\mathbf{6 . 0 6 7 9 7}$ & $\mathbf{8 . 6 6 0 1 5}$ & $\mathbf{1 . 1 0 8 3 2}$ & $\mathbf{0 . 9 8 0 6 3}$ & $\mathbf{0 . 6 9 7 5 2}$ & $\mathbf{1 . 6 1 0 6 7}$ & $\mathbf{1 . 7 5 4 8 5}$ & $\mathbf{0 . 8 0 3 8 5}$ \\
$\mathbf{2 0 0 1}$ & $\mathbf{6 . 3 3 6 5 2}$ & $\mathbf{8 . 9 2 5 0 7}$ & $\mathbf{1 . 1 3 1 1 3}$ & $\mathbf{0 . 9 9 1 8 6}$ & $\mathbf{0 . 7 1 2 5 6}$ & $\mathbf{1 . 6 4 6 8 8}$ & $\mathbf{1 . 8 1 2 0 6}$ & $\mathbf{0 . 8 7 3 9 6}$ \\
$\mathbf{2 0 0 2}$ & $\mathbf{6 . 3 6 4 9 5}$ & $\mathbf{9 . 0 8 3 3 9}$ & $\mathbf{1 . 1 2 4 6 7}$ & $\mathbf{0 . 9 7 3 5 4}$ & $\mathbf{0 . 6 7 9 6 4}$ & $\mathbf{1 . 6 4 4 5 4}$ & $\mathbf{1 . 7 9 3 8 5}$ & $\mathbf{0 . 8 9 3 4 7}$ \\
$\mathbf{2 0 0 3}$ & $\mathbf{6 . 4 1 8 4 2}$ & $\mathbf{9 . 3 8 9 3 5}$ & $\mathbf{1 . 1 1 3 0 5}$ & $\mathbf{0 . 9 4 7 0 0}$ & $\mathbf{0 . 6 5 8 7 1}$ & $\mathbf{1 . 6 6 4 1 2}$ & $\mathbf{1 . 7 5 7 5 7}$ & $\mathbf{0 . 8 9 4 6 6}$ \\
$\mathbf{2 0 0 4}$ & $\mathbf{6 . 5 4 6 4 1}$ & $\mathbf{9 . 7 9 4 2 2}$ & $\mathbf{1 . 1 3 5 8 3}$ & $\mathbf{0 . 9 5 2 3 5}$ & $\mathbf{0 . 6 5 5 1 0}$ & $\mathbf{1 . 6 9 5 2 6}$ & $\mathbf{1 . 7 5 6 3 6}$ & $\mathbf{0 . 9 3 4 0 6}$ \\
$\mathbf{2 0 0 5}$ & $\mathbf{6 . 6 8 4 1 6}$ & $\mathbf{1 0 . 1 1 9 9 3}$ & $\mathbf{1 . 1 5 2 4 5}$ & $\mathbf{0 . 9 5 6 2 1}$ & $\mathbf{0 . 6 6 6 2 8}$ & $\mathbf{1 . 7 3 8 4 6}$ & $\mathbf{1 . 7 6 1 0 8}$ & $\mathbf{0 . 9 8 4 0 1}$ \\
$\mathbf{2 0 0 6}$ & $\mathbf{6 . 8 0 5 0 6}$ & $\mathbf{1 0 . 5 2 4 4 5}$ & $\mathbf{1 . 1 8 3 7 7}$ & $\mathbf{0 . 9 7 3 7 4}$ & $\mathbf{0 . 6 8 8 8 7}$ & $\mathbf{1 . 7 9 5 7 2}$ & $\mathbf{1 . 7 9 4 4 1}$ & $\mathbf{1 . 0 8 6 2 7}$ \\
$\mathbf{2 0 0 7}$ & $\mathbf{6 . 9 7 1 8 7}$ & $\mathbf{1 0 . 9 6 2 8 6}$ & $\mathbf{1 . 1 6 8 3 1}$ & $\mathbf{0 . 9 5 3 5 9}$ & $\mathbf{0 . 6 7 2 2 9}$ & $\mathbf{1 . 7 9 5 1 1}$ & $\mathbf{1 . 7 7 8 6 5}$ & $\mathbf{1 . 0 5 7 5 7}$ \\
$\mathbf{2 0 0 8}$ & $\mathbf{6 . 9 9 9 3 9}$ & $\mathbf{1 1 . 2 5 8 5 4}$ & $\mathbf{1 . 2 0 7 4 1}$ & $\mathbf{0 . 9 8 4 3 3}$ & $\mathbf{0 . 7 1 3 7 2}$ & $\mathbf{1 . 8 3 0 6 6}$ & $\mathbf{1 . 8 4 9 2 7}$ & $\mathbf{1 . 1 6 1 5 4}$ \\
$\mathbf{2 0 0 9}$ & $\mathbf{6 . 8 6 3 8 0}$ & $\mathbf{1 1 . 4 0 6 6 4}$ & $\mathbf{1 . 1 7 3 9 9}$ & $\mathbf{0 . 9 4 2 3 2}$ & $\mathbf{0 . 6 8 1 2 0}$ & $\mathbf{1 . 7 6 6 7 0}$ & $\mathbf{1 . 7 9 3 3 1}$ & $\mathbf{1 . 0 8 4 1 8}$ \\
$\mathbf{2 0 1 0}$ & $\mathbf{7 . 0 9 6 0 2}$ & $\mathbf{1 1 . 6 8 1 0 9}$ & $\mathbf{1 . 2 1 2 6 3}$ & $\mathbf{0 . 9 7 0 2 9}$ & $\mathbf{0 . 7 0 3 9 0}$ & $\mathbf{1 . 7 9 1 2 4}$ & $\mathbf{1 . 8 2 6 4 7}$ & $\mathbf{1 . 1 2 8 9 8}$ \\
$\mathbf{2 0 1 1}$ & $\mathbf{7 . 2 2 2 7 7}$ & $\mathbf{1 1 . 9 7 4 6 6}$ & $\mathbf{1 . 3 2 3 7 8}$ & $\mathbf{1 . 0 5 4 8 5}$ & $\mathbf{0 . 7 8 8 4 5}$ & $\mathbf{1 . 8 8 9 6 2}$ & $\mathbf{1 . 9 2 9 2 2}$ & $\mathbf{1 . 3 2 1 3 5}$ \\
$\mathbf{2 0 1 2}$ & $\mathbf{7 . 3 7 1 8 8}$ & $\mathbf{1 2 . 2 7 4 4 0}$ & $\mathbf{1 . 3 8 7 5 0}$ & $\mathbf{1 . 0 9 6 1 3}$ & $\mathbf{0 . 8 2 8 8 1}$ & $\mathbf{1 . 9 5 3 9 7}$ & $\mathbf{1 . 9 8 3 1 6}$ & $\mathbf{1 . 4 2 4 4 8}$ \\
$\mathbf{2 0 1 3}$ & $\mathbf{7 . 5 3 3 3 9}$ & $\mathbf{1 2 . 3 9 7 7 4}$ & $\mathbf{1 . 3 8 0 7 2}$ & $\mathbf{1 . 0 8 6 6 2}$ & $\mathbf{0 . 8 1 2 1 7}$ & $\mathbf{1 . 9 6 2 9 4}$ & $\mathbf{1 . 9 8 7 3 4}$ & $\mathbf{1 . 4 4 4 2 8}$ \\
$\mathbf{2 0 1 4}$ & $\mathbf{7 . 7 1 1 0 0}$ & $\mathbf{1 2 . 6 6 6 6 0}$ & $\mathbf{1 . 3 7 7 4 3}$ & $\mathbf{1 . 0 8 1 3 1}$ & $\mathbf{0 . 7 9 5 3 1}$ & $\mathbf{1 . 9 7 3 2 6}$ & $\mathbf{2 . 0 0 2 4 1}$ & $\mathbf{1 . 4 7 3 6 4}$ \\
\hline
\end{tabular}

Table A3 Continued: Value Added Output Price Indexes $\mathrm{p}^{\mathrm{t}}$ and Input Price Indexes $\mathrm{w}_{1}{ }^{\mathrm{t}}$ for Labour and $\mathrm{w}_{2}{ }^{\mathrm{t}}-\mathrm{w}_{15}{ }^{\mathrm{t}}$ for Capital Services for the U.S. Noncorporate Nonfinancial Sector, 19602014

\begin{tabular}{|c|c|c|c|c|c|c|c|c|}
\hline Year & $\mathrm{w}_{8}{ }^{\mathrm{t}}$ & $\mathrm{wg}^{\mathrm{t}}$ & $w_{10}{ }^{t}$ & $w_{11}{ }^{t}$ & $w_{12}{ }^{t}$ & $w_{13}{ }^{t}$ & $\mathrm{w}_{14}{ }^{\mathrm{t}}$ & $\mathrm{w}_{15}{ }^{\mathrm{t}}$ \\
\hline 1960 & 0.09072 & 0.08984 & 0.09213 & 0.06938 & 0.02148 & 0.07296 & 0.07312 & 0.05886 \\
\hline 1961 & 0.09506 & 0.09238 & 0.09872 & 0.07675 & 0.02848 & 0.07264 & 0.07942 & 0.06610 \\
\hline 1962 & 0.09886 & 0.09617 & 0.10338 & 0.08287 & 0.03465 & 0.07829 & 0.08402 & 0.07099 \\
\hline 1963 & 0.10042 & 0.10002 & 0.10491 & 0.08980 & 0.03791 & 0.08101 & 0.08642 & 0.07353 \\
\hline 1964 & 0.10822 & 0.10590 & 0.10671 & 0.09425 & 0.04452 & 0.08560 & 0.08805 & 0.07812 \\
\hline 1965 & 0.11990 & 0.11811 & 0.12094 & 0.09570 & 0.05982 & 0.09755 & 0.09763 & 0.08965 \\
\hline 1966 & 0.13161 & 0.12746 & 0.13017 & 0.10773 & 0.06890 & 0.10378 & 0.10399 & 0.10018 \\
\hline 1967 & 0.13386 & 0.12812 & 0.13047 & 0.10692 & 0.07038 & 0.10218 & 0.10394 & 0.10034 \\
\hline 1968 & 0.13766 & 0.13266 & 0.13612 & 0.10840 & 0.07836 & 0.10709 & 0.10840 & 0.10545 \\
\hline 1969 & 0.13969 & 0.13350 & 0.13734 & 0.10482 & 0.08148 & 0.10625 & 0.10749 & 0.10514 \\
\hline 1970 & 0.13989 & 0.13318 & 0.13726 & 0.09503 & 0.08352 & 0.10269 & 0.10516 & 0.10338 \\
\hline 1971 & 0.15276 & 0.14215 & 0.14820 & 0.09693 & 0.09623 & 0.10931 & 0.11056 & 0.11022 \\
\hline 1972 & 0.17157 & 0.16120 & 0.16771 & 0.11916 & 0.11818 & 0.12582 & 0.12331 & 0.12547 \\
\hline 1973 & 0.21048 & 0.19441 & 0.20087 & 0.16222 & 0.16111 & 0.15963 & 0.14975 & 0.15367 \\
\hline 1974 & 0.20619 & 0.19081 & 0.19644 & 0.14346 & 0.13619 & 0.14857 & 0.14443 & 0.14403 \\
\hline 1975 & 0.22286 & 0.20855 & 0.20823 & 0.14216 & 0.14520 & 0.15712 & 0.15241 & 0.15198 \\
\hline 1976 & 0.24167 & 0.23149 & 0.22752 & 0.15871 & 0.16747 & $\mathbf{0 . 1 7 6 3 7}$ & 0.16491 & 0.16659 \\
\hline 1977 & 0.26097 & 0.24898 & 0.24405 & 0.17589 & 0.17862 & 0.19110 & 0.17320 & 0.17627 \\
\hline 1978 & 0.29480 & 0.27448 & 0.27715 & 0.20855 & 0.21803 & 0.21831 & 0.18958 & 0.19512 \\
\hline 1979 & 0.32396 & 0.29523 & 0.30019 & 0.21885 & 0.23318 & 0.23535 & 0.20505 & 0.20687 \\
\hline 1980 & 0.32133 & 0.28985 & 0.29581 & 0.17490 & 0.17918 & 0.21942 & 0.19834 & 0.19733 \\
\hline 1981 & 0.35111 & $\mathbf{0 . 3 0 5 3 7}$ & 0.31073 & 0.20339 & 0.18103 & 0.22887 & 0.20795 & 0.20617 \\
\hline 1982 & 0.35116 & 0.29705 & 0.30339 & 0.18084 & 0.15976 & 0.21273 & 0.19442 & 0.19669 \\
\hline 1983 & 0.34869 & 0.30429 & 0.31264 & 0.19397 & 0.18691 & 0.21737 & 0.19505 & 0.19988 \\
\hline 1984 & 0.41227 & 0.37015 & 0.37854 & 0.31695 & 0.31778 & 0.30091 & 0.24344 & 0.25197 \\
\hline 1985 & 0.44322 & 0.39791 & 0.40994 & 0.37602 & 0.35963 & 0.33931 & 0.26097 & 0.27433 \\
\hline 1986 & 0.44501 & 0.39257 & 0.40744 & 0.48061 & 0.40255 & 0.34018 & 0.23306 & 0.26840 \\
\hline 1987 & 0.45613 & 0.40177 & 0.42784 & 0.50342 & 0.39231 & 0.35387 & 0.23012 & 0.27049 \\
\hline 1988 & 0.49275 & 0.43272 & 0.46325 & 0.58695 & 0.44723 & 0.40169 & 0.25508 & 0.29233 \\
\hline 1989 & 0.50591 & 0.45954 & 0.47642 & 0.66349 & 0.49404 & 0.41777 & 0.27652 & 0.29909 \\
\hline 1990 & 0.50911 & 0.46847 & 0.46526 & 0.72205 & 0.49003 & 0.40575 & 0.27057 & 0.29461 \\
\hline 1991 & 0.47602 & 0.44283 & 0.43609 & 0.70229 & 0.47826 & 0.37726 & 0.24522 & 0.27355 \\
\hline 1992 & 0.51076 & 0.48413 & 0.47672 & 0.86271 & 0.55879 & 0.44591 & 0.26449 & 0.30692 \\
\hline 1993 & 0.51095 & 0.47477 & 0.48665 & 0.89740 & 0.56382 & 0.44040 & 0.25523 & 0.30042 \\
\hline 1994 & 0.53294 & 0.49626 & 0.50510 & 1.00655 & 0.62121 & 0.46437 & 0.26870 & 0.31309 \\
\hline 1995 & 0.56335 & 0.52162 & 0.52950 & 1.03423 & 0.66920 & 0.48532 & 0.28290 & 0.32625 \\
\hline 1996 & 0.62861 & 0.58282 & 0.58647 & 1.12767 & 0.76565 & 0.56837 & 0.32232 & 0.37511 \\
\hline 1997 & 0.66856 & 0.60585 & 0.61740 & 1.16206 & 0.79336 & 0.61442 & 0.32750 & 0.39663 \\
\hline 1998 & 0.72867 & 0.65763 & 0.67889 & 1.29081 & 0.84876 & 0.69721 & 0.34387 & 0.43271 \\
\hline 1999 & 0.76565 & 0.68368 & 0.72428 & 1.47920 & 0.89927 & 0.77795 & 0.35011 & 0.45535 \\
\hline 2000 & 0.80768 & 0.71105 & 0.76233 & 1.66345 & 0.89806 & 0.85925 & 0.36292 & 0.47644 \\
\hline 2001 & 0.87725 & 0.76629 & 0.82632 & 2.02110 & 0.93616 & 0.99372 & 0.38508 & 0.51789 \\
\hline
\end{tabular}




\begin{tabular}{lllllllll}
\hline $\mathbf{2 0 0 2}$ & $\mathbf{0 . 8 9 6 0 9}$ & $\mathbf{0 . 7 7 2 6 8}$ & $\mathbf{0 . 8 4 7 8 8}$ & $\mathbf{2 . 2 1 4 2 0}$ & $\mathbf{0 . 9 0 9 9 6}$ & $\mathbf{1 . 0 4 5 3 7}$ & $\mathbf{0 . 3 6 8 7 9}$ & $\mathbf{0 . 5 1 6 1 1}$ \\
$\mathbf{2 0 0 3}$ & $\mathbf{0 . 8 9 5 4 1}$ & $\mathbf{0 . 7 6 8 4 3}$ & $\mathbf{0 . 8 5 1 1 5}$ & $\mathbf{2 . 3 5 2 7 2}$ & $\mathbf{0 . 8 9 4 2 0}$ & $\mathbf{1 . 0 8 7 9 6}$ & $\mathbf{0 . 3 6 5 9 5}$ & $\mathbf{0 . 5 0 8 7 7}$ \\
$\mathbf{2 0 0 4}$ & $\mathbf{0 . 9 3 5 9 5}$ & $\mathbf{0 . 7 9 9 1 8}$ & $\mathbf{0 . 8 8 5 5 6}$ & $\mathbf{2 . 6 2 0 2 8}$ & $\mathbf{0 . 9 2 1 0 6}$ & $\mathbf{1 . 1 9 5 0 6}$ & $\mathbf{0 . 3 8 1 5 6}$ & $\mathbf{0 . 5 2 3 5 7}$ \\
$\mathbf{2 0 0 5}$ & $\mathbf{0 . 9 8 7 6 4}$ & $\mathbf{0 . 8 2 6 6 5}$ & $\mathbf{0 . 9 1 0 4 6}$ & $\mathbf{2 . 7 8 5 3 3}$ & $\mathbf{0 . 9 7 6 5 1}$ & $\mathbf{1 . 2 1 8 0 8}$ & $\mathbf{0 . 3 8 3 5 9}$ & $\mathbf{0 . 5 2 2 1 5}$ \\
$\mathbf{2 0 0 6}$ & $\mathbf{1 . 0 9 1 9 0}$ & $\mathbf{0 . 8 7 8 2 1}$ & $\mathbf{0 . 9 8 3 7 6}$ & $\mathbf{3 . 0 0 9 9 9}$ & $\mathbf{1 . 1 2 3 1 1}$ & $\mathbf{1 . 3 1 6 3 2}$ & $\mathbf{0 . 4 0 6 1 0}$ & $\mathbf{0 . 5 5 3 1 7}$ \\
$\mathbf{2 0 0 7}$ & $\mathbf{1 . 0 6 8 4 9}$ & $\mathbf{0 . 8 6 3 0 2}$ & $\mathbf{0 . 9 4 8 3 7}$ & $\mathbf{2 . 7 4 4 1 7}$ & $\mathbf{1 . 0 6 8 0 6}$ & $\mathbf{1 . 2 0 0 0 8}$ & $\mathbf{0 . 3 7 7 7 1}$ & $\mathbf{0 . 5 1 9 7 6}$ \\
$\mathbf{2 0 0 8}$ & $\mathbf{1 . 1 7 4 2 4}$ & $\mathbf{0 . 9 6 5 5 6}$ & $\mathbf{1 . 0 2 4 8 4}$ & $\mathbf{2 . 9 0 8 1 7}$ & $\mathbf{1 . 2 0 7 9 6}$ & $\mathbf{1 . 2 7 1 8 0}$ & $\mathbf{0 . 4 3 9 6 6}$ & $\mathbf{0 . 5 7 4 8 1}$ \\
$\mathbf{2 0 0 9}$ & $\mathbf{1 . 0 9 6 7 1}$ & $\mathbf{0 . 9 3 3 8 3}$ & $\mathbf{0 . 9 4 3 9 2}$ & $\mathbf{2 . 3 2 7 8 4}$ & $\mathbf{1 . 0 9 7 9 0}$ & $\mathbf{1 . 0 1 8 0 1}$ & $\mathbf{0 . 3 8 1 3 6}$ & $\mathbf{0 . 5 3 4 0 9}$ \\
$\mathbf{2 0 1 0}$ & $\mathbf{1 . 1 4 2 3 6}$ & $\mathbf{0 . 9 7 3 7 3}$ & $\mathbf{0 . 9 9 8 1 7}$ & $\mathbf{2 . 4 5 2 4 1}$ & $\mathbf{1 . 2 2 8 5 5}$ & $\mathbf{1 . 0 7 2 4 9}$ & $\mathbf{0 . 4 1 1 5 2}$ & $\mathbf{0 . 5 7 7 8 0}$ \\
$\mathbf{2 0 1 1}$ & $\mathbf{1 . 3 4 1 8 5}$ & $\mathbf{1 . 1 8 2 7 3}$ & $\mathbf{1 . 1 6 4 7 7}$ & $\mathbf{2 . 6 8 5 6 6}$ & $\mathbf{1 . 6 3 4 2 2}$ & $\mathbf{1 . 1 7 4 4 9}$ & $\mathbf{0 . 5 1 0 9 9}$ & $\mathbf{0 . 6 9 2 1 0}$ \\
$\mathbf{2 0 1 2}$ & $\mathbf{1 . 4 5 0 6 2}$ & $\mathbf{1 . 2 9 1 3 1}$ & $\mathbf{1 . 2 3 4 9 3}$ & $\mathbf{2 . 7 4 0 4 0}$ & $\mathbf{1 . 8 8 2 2 6}$ & $\mathbf{1 . 1 9 8 4 3}$ & $\mathbf{0 . 5 6 1 1 2}$ & $\mathbf{0 . 7 4 8 1 6}$ \\
$\mathbf{2 0 1 3}$ & $\mathbf{1 . 4 6 9 4 7}$ & $\mathbf{1 . 2 9 8 0 9}$ & $\mathbf{1 . 2 5 8 3 6}$ & $\mathbf{3 . 0 3 3 1 5}$ & $\mathbf{2 . 0 4 0 3 5}$ & $\mathbf{1 . 3 2 6 4 5}$ & $\mathbf{0 . 5 4 9 6 1}$ & $\mathbf{0 . 7 4 2 5 8}$ \\
$\mathbf{2 0 1 4}$ & $\mathbf{1 . 4 9 8 4 3}$ & $\mathbf{1 . 2 9 2 3 7}$ & $\mathbf{1 . 2 8 1 9 1}$ & $\mathbf{3 . 3 0 0 8 1}$ & $\mathbf{2 . 1 1 5 2 3}$ & $\mathbf{1 . 4 4 3 5 1}$ & $\mathbf{0 . 5 2 7 7 8}$ & $\mathbf{0 . 7 3 4 5 7}$ \\
\hline
\end{tabular}

Table A4: Real Value Added Output Volumes $y^{t}$ and Input Quantities $x_{1}{ }^{t}$ for Labour and $x_{2}{ }^{t}-x_{15}{ }^{t}$ for Capital Services for the U.S. Corporate Nonfinancial Sector, 1960-2014 in Billions of 1960 U.S. Dollars

\begin{tabular}{|c|c|c|c|c|c|c|c|c|}
\hline Year & $y^{t}$ & $\mathbf{x}_{1}{ }^{t}$ & $\mathbf{x}_{2}{ }^{t}$ & $\mathbf{x}_{3}{ }^{t}$ & $\mathrm{x}_{4}{ }^{\mathrm{t}}$ & $\mathbf{x}_{5}{ }^{\mathrm{t}}$ & $x_{6}{ }^{t}$ & $\mathbf{x}_{7}{ }^{\mathrm{t}}$ \\
\hline 1960 & 107.40 & 76.63 & 27.20 & 14.50 & 1.70 & 2.30 & 2.60 & 32.30 \\
\hline 1961 & 108.46 & 74.57 & 27.09 & 14.56 & 1.77 & 2.34 & 2.65 & 33.24 \\
\hline 1962 & 110.81 & 73.13 & 26.87 & 14.55 & 1.82 & 2.42 & 2.76 & 34.14 \\
\hline 1963 & 112.34 & 71.47 & 27.07 & 14.73 & 1.89 & 2.48 & 2.83 & 35.23 \\
\hline 1964 & 116.05 & 71.22 & 27.60 & 15.08 & 1.97 & 2.52 & 2.88 & 36.38 \\
\hline 1965 & 120.26 & 70.24 & 28.43 & 15.71 & 2.00 & 2.52 & 2.88 & 37.54 \\
\hline 1966 & 124.31 & 69.03 & 29.71 & 16.53 & 2.06 & 2.53 & 2.88 & 38.85 \\
\hline 1967 & 123.63 & 67.32 & 31.28 & 17.67 & 2.17 & 2.53 & 2.86 & 39.97 \\
\hline 1968 & 124.68 & 65.69 & 32.39 & 18.46 & 2.25 & 2.50 & 2.81 & 40.99 \\
\hline 1969 & 125.83 & 65.08 & 33.35 & 19.20 & 2.34 & 2.48 & 2.79 & 41.84 \\
\hline 1970 & 125.38 & 63.31 & 34.51 & 20.17 & 2.42 & 2.47 & 2.76 & 42.81 \\
\hline 1971 & 126.85 & 61.94 & 35.59 & 21.08 & 2.53 & 2.46 & 2.73 & 43.77 \\
\hline 1972 & 133.69 & 61.82 & 36.64 & 21.81 & 2.61 & 2.44 & 2.68 & 44.60 \\
\hline 1973 & 149.72 & 63.69 & 37.95 & 22.40 & 2.71 & 2.40 & 2.61 & 45.33 \\
\hline 1974 & 143.99 & 63.96 & 40.59 & 23.39 & 2.83 & 2.35 & 2.56 & 46.43 \\
\hline 1975 & 139.86 & 62.50 & 42.21 & 23.93 & 2.99 & 2.29 & 2.49 & 47.57 \\
\hline 1976 & 140.71 & 62.63 & 43.00 & 24.13 & 3.15 & 2.24 & 2.43 & 48.64 \\
\hline 1977 & 141.63 & 63.82 & 43.92 & 24.27 & 3.32 & 2.24 & 2.44 & 49.68 \\
\hline 1978 & 149.77 & 65.87 & 45.30 & 24.76 & 3.56 & 2.22 & 2.40 & 50.80 \\
\hline 1979 & 149.54 & 67.44 & 47.77 & 25.85 & 3.60 & 2.28 & 2.47 & 52.38 \\
\hline 1980 & 147.36 & 67.40 & 50.36 & 27.27 & 3.82 & 2.42 & 2.60 & 54.10 \\
\hline 1981 & 146.33 & 67.21 & 50.94 & 28.00 & 3.84 & 2.56 & 2.72 & 55.95 \\
\hline 1982 & 143.46 & 67.12 & 51.59 & 29.07 & 3.90 & 2.74 & 2.88 & 58.48 \\
\hline 1983 & 141.09 & 68.39 & 50.69 & 29.47 & 3.81 & 2.85 & 2.95 & 60.50 \\
\hline 1984 & 161.22 & 70.75 & 49.94 & 29.54 & 3.68 & 3.00 & 3.05 & 61.63 \\
\hline 1985 & 165.94 & 70.79 & 50.13 & 30.42 & 3.57 & 3.21 & 3.25 & 62.98 \\
\hline 1986 & 172.81 & 70.85 & 49.82 & 31.50 & 3.89 & 3.43 & 3.50 & 64.47 \\
\hline 1987 & 172.81 & 71.81 & 49.35 & 32.35 & 4.03 & 3.71 & 3.79 & 65.57 \\
\hline 1988 & 179.53 & 73.01 & 49.00 & 33.23 & 3.99 & 4.10 & 4.20 & 66.75 \\
\hline 1989 & 181.42 & 74.83 & 49.20 & 34.47 & 3.99 & 4.47 & 4.63 & 67.90 \\
\hline 1990 & 181.67 & 75.52 & 49.35 & 35.47 & 3.94 & 4.85 & 5.07 & 68.71 \\
\hline 1991 & 176.04 & 75.76 & 48.57 & 35.06 & 3.99 & 5.15 & 5.42 & 69.02 \\
\hline 1992 & 184.98 & 74.09 & 47.47 & 34.38 & 3.98 & 5.45 & 5.76 & 68.89 \\
\hline 1993 & 187.72 & 77.42 & 46.54 & 33.61 & 4.03 & 5.79 & 6.09 & 68.37 \\
\hline 1994 & 195.38 & 78.99 & 46.61 & 33.56 & 4.12 & 6.11 & 6.39 & 68.28 \\
\hline 1995 & 194.55 & 79.86 & 47.54 & 34.06 & 4.19 & 6.38 & 6.68 & 68.16 \\
\hline 1996 & 199.49 & 80.39 & 48.54 & 34.83 & 4.27 & 6.61 & 7.01 & 68.22 \\
\hline 1997 & 205.21 & 81.85 & 49.60 & 35.90 & 4.36 & 6.85 & 7.39 & 68.39 \\
\hline 1998 & 217.13 & 81.78 & 51.18 & 37.36 & 4.47 & 7.26 & 7.91 & 68.54 \\
\hline 1999 & 227.58 & 81.46 & 52.23 & 40.03 & 4.62 & 7.79 & 9.03 & 68.83 \\
\hline 2000 & 234.13 & 82.32 & 52.71 & 44.11 & 4.88 & 8.29 & 10.69 & 69.05 \\
\hline 2001 & 258.36 & 93.06 & 52.93 & 49.54 & 5.29 & 8.65 & 12.73 & 69.31 \\
\hline 2002 & 268.19 & 94.87 & 52.36 & 54.71 & 5.77 & 8.71 & 14.72 & 69.29 \\
\hline
\end{tabular}




\begin{tabular}{rrrrrrrrr}
\hline $\mathbf{2 0 0 3}$ & $\mathbf{2 8 0 . 5 2}$ & $\mathbf{9 9 . 4 9}$ & $\mathbf{5 1 . 8 5}$ & $\mathbf{5 9 . 7 0}$ & $\mathbf{6 . 2 8}$ & $\mathbf{8 . 7 3}$ & $\mathbf{1 6 . 8 3}$ & $\mathbf{6 8 . 9 6}$ \\
$\mathbf{2 0 0 4}$ & $\mathbf{2 9 8 . 4 4}$ & $\mathbf{1 0 4 . 4 8}$ & $\mathbf{5 1 . 7 2}$ & $\mathbf{6 4 . 3 1}$ & $\mathbf{6 . 7 7}$ & $\mathbf{8 . 8 2}$ & $\mathbf{1 8 . 6 7}$ & $\mathbf{6 8 . 6 3}$ \\
$\mathbf{2 0 0 5}$ & $\mathbf{3 1 2 . 4 7}$ & $\mathbf{1 0 8 . 9 7}$ & $\mathbf{5 2 . 3 2}$ & $\mathbf{6 9 . 5 3}$ & $\mathbf{7 . 1 9}$ & $\mathbf{8 . 9 3}$ & $\mathbf{2 0 . 3 8}$ & $\mathbf{6 8 . 2 1}$ \\
$\mathbf{2 0 0 6}$ & $\mathbf{3 3 6 . 9 7}$ & $\mathbf{1 1 4 . 9 7}$ & $\mathbf{5 3 . 4 9}$ & $\mathbf{7 5 . 4 3}$ & $\mathbf{7 . 7 1}$ & $\mathbf{9 . 1 5}$ & $\mathbf{2 2 . 1 8}$ & $\mathbf{6 7 . 7 5}$ \\
$\mathbf{2 0 0 7}$ & $\mathbf{3 3 7 . 9 7}$ & $\mathbf{1 1 8 . 8 5}$ & $\mathbf{5 4 . 7 9}$ & $\mathbf{8 1 . 9 9}$ & $\mathbf{8 . 3 9}$ & $\mathbf{9 . 3 3}$ & $\mathbf{2 3 . 8 7}$ & $\mathbf{6 7 . 3 3}$ \\
$\mathbf{2 0 0 8}$ & $\mathbf{3 5 3 . 5 3}$ & $\mathbf{1 1 6 . 8 7}$ & $\mathbf{5 5 . 8 7}$ & $\mathbf{8 8 . 7 8}$ & $\mathbf{9 . 1 7}$ & $\mathbf{9 . 4 5}$ & $\mathbf{2 5 . 4 5}$ & $\mathbf{6 7 . 0 9}$ \\
$\mathbf{2 0 0 9}$ & $\mathbf{3 3 8 . 1 5}$ & $\mathbf{1 1 1 . 4 4}$ & $\mathbf{5 5 . 0 3}$ & $\mathbf{9 4 . 9 5}$ & $\mathbf{9 . 7 8}$ & $\mathbf{9 . 3 1}$ & $\mathbf{2 7 . 0 9}$ & $\mathbf{6 7 . 0 9}$ \\
$\mathbf{2 0 1 0}$ & $\mathbf{3 3 7 . 5 8}$ & $\mathbf{1 1 0 . 5 9}$ & $\mathbf{5 3 . 2 7}$ & $\mathbf{9 6 . 5 0}$ & $\mathbf{1 0 . 2 4}$ & $\mathbf{9 . 2 2}$ & $\mathbf{2 8 . 2 1}$ & $\mathbf{6 6 . 7 5}$ \\
$\mathbf{2 0 1 1}$ & $\mathbf{3 5 8 . 9 9}$ & $\mathbf{1 1 0 . 5 9}$ & $\mathbf{5 2 . 4 6}$ & $\mathbf{9 9 . 7 8}$ & $\mathbf{1 0 . 6 8}$ & $\mathbf{9 . 2 0}$ & $\mathbf{2 9 . 2 3}$ & $\mathbf{6 6 . 0 8}$ \\
$\mathbf{2 0 1 2}$ & $\mathbf{3 7 1 . 9 9}$ & $\mathbf{1 1 2 . 9 8}$ & $\mathbf{5 2 . 4 2}$ & $\mathbf{1 0 3 . 8 3}$ & $\mathbf{1 1 . 2 2}$ & $\mathbf{9 . 1 9}$ & $\mathbf{3 0 . 1 8}$ & $\mathbf{6 5 . 4 4}$ \\
$\mathbf{2 0 1 3}$ & $\mathbf{3 7 6 . 9 6}$ & $\mathbf{1 1 3 . 8 0}$ & $\mathbf{5 3 . 7 0}$ & $\mathbf{1 0 9 . 5 0}$ & $\mathbf{1 1 . 8 3}$ & $\mathbf{9 . 3 3}$ & $\mathbf{3 1 . 2 5}$ & $\mathbf{6 4 . 9 3}$ \\
$\mathbf{2 0 1 4}$ & $\mathbf{3 8 4 . 6 8}$ & $\mathbf{1 1 6 . 0 8}$ & $\mathbf{5 5 . 1 1}$ & $\mathbf{1 1 5 . 1 6}$ & $\mathbf{1 2 . 3 5}$ & $\mathbf{9 . 4 6}$ & $\mathbf{3 2 . 3 1}$ & $\mathbf{6 4 . 5 4}$ \\
\hline
\end{tabular}

Table A4 Continued: Real Value Added Output Volumes $\mathbf{y}^{\mathrm{t}}$ and Input Quantities $\mathrm{x}_{1}{ }^{\mathrm{t}}$ for Labour and $\mathbf{x}_{2}{ }^{t}-x_{15}{ }^{t}$ for Capital Services for the U.S. Corporate Nonfinancial Sector, 1960-2014 in Billions of 1960 U.S. Dollars

\begin{tabular}{|c|c|c|c|c|c|c|c|c|}
\hline Year & $\mathrm{x}_{8}{ }^{t}$ & $\mathrm{x}_{9}{ }^{\mathrm{t}}$ & $\mathrm{x}_{10}{ }^{\mathrm{t}}$ & $\mathrm{x}_{11}{ }^{\mathrm{t}}$ & $\mathrm{x}_{12}{ }^{\mathrm{t}}$ & $\mathrm{x}_{13}{ }^{\mathrm{t}}$ & $x_{14}{ }^{t}$ & $\mathrm{x}_{15}{ }^{\mathrm{t}}$ \\
\hline 1960 & 19.70 & 3.20 & 45.00 & 63.77 & 86.77 & 17.02 & 34.10 & 18.70 \\
\hline 1961 & 20.38 & 3.33 & 45.45 & 64.31 & 86.64 & 17.01 & 34.99 & 17.74 \\
\hline 1962 & 21.12 & 3.45 & 46.02 & 64.90 & 86.58 & 17.61 & 36.48 & 17.82 \\
\hline 1963 & 21.97 & 3.57 & 46.72 & 65.52 & 86.46 & 18.19 & 38.09 & 17.58 \\
\hline 1964 & 22.88 & 3.69 & 47.36 & 65.78 & 86.17 & 18.85 & 36.49 & 17.16 \\
\hline 1965 & 23.92 & 3.81 & 48.12 & 67.43 & 85.66 & 19.92 & 35.44 & 17.02 \\
\hline 1966 & 25.27 & 3.94 & 48.71 & 69.41 & 84.93 & 20.94 & 38.61 & 16.86 \\
\hline 1967 & 26.49 & 4.07 & 49.59 & 71.73 & 83.99 & 21.90 & 39.10 & 16.34 \\
\hline 1968 & 27.54 & 4.24 & 50.22 & 73.99 & 82.86 & 22.57 & 39.34 & 16.02 \\
\hline 1969 & 28.35 & 4.40 & 54.30 & 76.53 & 81.57 & 23.74 & 41.72 & 15.53 \\
\hline 1970 & 29.43 & 4.57 & 59.74 & 81.09 & 80.19 & 24.92 & 43.15 & 14.91 \\
\hline 1971 & 30.46 & 4.74 & 64.77 & 83.65 & 78.85 & 26.02 & 40.99 & 15.40 \\
\hline 1972 & 31.60 & 4.91 & 71.13 & 84.66 & 77.67 & 28.13 & 45.09 & 16.10 \\
\hline 1973 & 32.83 & 5.08 & 79.45 & 85.53 & 76.80 & 30.04 & 50.09 & 16.93 \\
\hline 1974 & 34.43 & 5.24 & 86.03 & 87.07 & 76.38 & 31.89 & 53.93 & 19.93 \\
\hline 1975 & 35.41 & 5.38 & 91.83 & 90.47 & 76.46 & 32.13 & 45.19 & 18.83 \\
\hline 1976 & 36.20 & 5.62 & 93.47 & 92.31 & 76.83 & 31.12 & 46.28 & 18.13 \\
\hline 1977 & 37.04 & 5.86 & 94.66 & 91.70 & 77.23 & 31.06 & 43.97 & 19.05 \\
\hline 1978 & 37.90 & 6.16 & 96.56 & 90.86 & 77.37 & 31.95 & 46.08 & 20.52 \\
\hline 1979 & 39.21 & 6.27 & 99.05 & 91.23 & 77.07 & 33.28 & 52.71 & 22.13 \\
\hline 1980 & 41.47 & 6.51 & 102.64 & 91.57 & 76.46 & 34.83 & 54.55 & 23.83 \\
\hline 1981 & 44.00 & 6.62 & 105.11 & 93.63 & 75.70 & 36.14 & 50.01 & 23.75 \\
\hline 1982 & 47.57 & 6.75 & 107.05 & 96.61 & 74.99 & 38.04 & 47.17 & 23.42 \\
\hline 1983 & 51.14 & 6.75 & 107.94 & 99.82 & 74.46 & 38.72 & 48.50 & 24.37 \\
\hline 1984 & 53.73 & 6.68 & 110.06 & 100.61 & 74.08 & 38.96 & 45.48 & 27.41 \\
\hline 1985 & 57.29 & 6.63 & 112.67 & 101.64 & 73.77 & 41.00 & 47.38 & 29.13 \\
\hline 1986 & 61.80 & 6.77 & 115.83 & 102.71 & 73.45 & 43.03 & 47.90 & 34.14 \\
\hline 1987 & 65.52 & 6.84 & 119.60 & 102.38 & 73.06 & 44.12 & 45.12 & 34.85 \\
\hline 1988 & 69.29 & 6.84 & 122.27 & 101.82 & 72.57 & 45.75 & 44.79 & 33.27 \\
\hline 1989 & 72.93 & 6.81 & 124.30 & 101.70 & 72.03 & 47.04 & 44.00 & 35.00 \\
\hline 1990 & 75.95 & 6.79 & 126.23 & 101.63 & 71.51 & 48.25 & 44.52 & 34.39 \\
\hline 1991 & 77.46 & 6.83 & 127.62 & 103.14 & 71.12 & 48.27 & 45.22 & 34.00 \\
\hline 1992 & 77.91 & 6.87 & 128.22 & 103.97 & 70.94 & 46.97 & 42.45 & 34.28 \\
\hline 1993 & $\mathbf{7 7 . 5 0}$ & 6.88 & 128.73 & 103.04 & 71.03 & 46.36 & 44.77 & 36.36 \\
\hline 1994 & 77.77 & 6.91 & 129.61 & 102.65 & 71.29 & 46.78 & 43.70 & 36.50 \\
\hline 1995 & 77.86 & 6.90 & 129.83 & 101.89 & 71.61 & 47.54 & 45.16 & 39.43 \\
\hline 1996 & 78.31 & 6.93 & 131.92 & 102.55 & 71.89 & 47.71 & 42.95 & 42.60 \\
\hline 1997 & 78.88 & 7.00 & 133.91 & 102.57 & 72.02 & 48.65 & 53.99 & 46.89 \\
\hline 1998 & 79.64 & 7.06 & 136.11 & 102.68 & 71.95 & 50.69 & 55.73 & 53.98 \\
\hline 1999 & 80.81 & 7.15 & 138.17 & 102.33 & 71.67 & 52.96 & 53.40 & 63.73 \\
\hline 2000 & 82.20 & 7.26 & 140.65 & 101.97 & 71.25 & 55.04 & 54.39 & 74.91 \\
\hline 2001 & 83.79 & 7.53 & 142.87 & 102.66 & 70.73 & $\mathbf{5 7 . 5 3}$ & 56.07 & 88.97 \\
\hline 2002 & 85.18 & 7.88 & 145.02 & 103.10 & 70.19 & 60.05 & 54.41 & 89.45 \\
\hline 2003 & 86.36 & 8.09 & 147.25 & 100.68 & 69.70 & 61.82 & 55.88 & 91.22 \\
\hline 2004 & 87.01 & 8.28 & 149.74 & 99.21 & 69.25 & 63.07 & 58.27 & 99.60 \\
\hline
\end{tabular}




\begin{tabular}{rrrrrrrrr}
\hline $\mathbf{2 0 0 5}$ & $\mathbf{8 7 . 6 7}$ & $\mathbf{8 . 3 9}$ & $\mathbf{1 5 2 . 0 3}$ & $\mathbf{9 7 . 9 9}$ & $\mathbf{6 8 . 8 0}$ & $\mathbf{6 6 . 7 9}$ & $\mathbf{5 8 . 3 7}$ & $\mathbf{1 1 4 . 8 1}$ \\
$\mathbf{2 0 0 6}$ & $\mathbf{8 8 . 3 6}$ & $\mathbf{8 . 4 9}$ & $\mathbf{1 5 3 . 3 4}$ & $\mathbf{9 5 . 9 4}$ & $\mathbf{6 8 . 3 5}$ & $\mathbf{7 2 . 1 7}$ & $\mathbf{5 8 . 8 9}$ & $\mathbf{1 3 0 . 0 2}$ \\
$\mathbf{2 0 0 7}$ & $\mathbf{9 0 . 0 3}$ & $\mathbf{8 . 6 2}$ & $\mathbf{1 5 5 . 6 3}$ & $\mathbf{9 5 . 5 7}$ & $\mathbf{6 7 . 8 7}$ & $\mathbf{7 6 . 2 4}$ & $\mathbf{5 8 . 7 3}$ & $\mathbf{1 4 5 . 1 0}$ \\
$\mathbf{2 0 0 8}$ & $\mathbf{9 2 . 4 7}$ & $\mathbf{8 . 9 0}$ & $\mathbf{1 5 7 . 2 4}$ & $\mathbf{9 6 . 7 7}$ & $\mathbf{6 7 . 4 4}$ & $\mathbf{7 8 . 3 9}$ & $\mathbf{5 7 . 0 7}$ & $\mathbf{1 5 4 . 8 9}$ \\
$\mathbf{2 0 0 9}$ & $\mathbf{9 5 . 3 4}$ & $\mathbf{9 . 1 7}$ & $\mathbf{1 5 7 . 8 3}$ & $\mathbf{9 8 . 6 8}$ & $\mathbf{6 7 . 1 2}$ & $\mathbf{7 9 . 6 8}$ & $\mathbf{6 0 . 2 4}$ & $\mathbf{1 5 3 . 1 0}$ \\
$\mathbf{2 0 1 0}$ & $\mathbf{9 6 . 7 2}$ & $\mathbf{9 . 5 8}$ & $\mathbf{1 5 7 . 4 5}$ & $\mathbf{9 9 . 6 5}$ & $\mathbf{6 6 . 9 6}$ & $\mathbf{7 4 . 6 4}$ & $\mathbf{5 5 . 1 8}$ & $\mathbf{1 5 3 . 0 3}$ \\
$\mathbf{2 0 1 1}$ & $\mathbf{9 7 . 1 1}$ & $\mathbf{9 . 8 9}$ & $\mathbf{1 5 6 . 3 4}$ & $\mathbf{1 0 0 . 2 9}$ & $\mathbf{6 7 . 0 1}$ & $\mathbf{7 3 . 6 9}$ & $\mathbf{5 9 . 4 4}$ & $\mathbf{1 5 1 . 1 2}$ \\
$\mathbf{2 0 1 2}$ & $\mathbf{9 7 . 5 8}$ & $\mathbf{1 0 . 1 9}$ & $\mathbf{1 5 5 . 4 3}$ & $\mathbf{1 0 1 . 4 1}$ & $\mathbf{6 7 . 2 8}$ & $\mathbf{7 3 . 3 5}$ & $\mathbf{6 3 . 5 9}$ & $\mathbf{1 5 2 . 2 8}$ \\
$\mathbf{2 0 1 3}$ & $\mathbf{9 8 . 3 5}$ & $\mathbf{1 0 . 6 4}$ & $\mathbf{1 5 4 . 6 6}$ & $\mathbf{1 0 1 . 6 8}$ & $\mathbf{6 7 . 8 7}$ & $\mathbf{7 4 . 1 3}$ & $\mathbf{6 2 . 5 0}$ & $\mathbf{1 6 0 . 9 4}$ \\
$\mathbf{2 0 1 4}$ & $\mathbf{9 9 . 3 0}$ & $\mathbf{1 0 . 9 3}$ & $\mathbf{1 5 4 . 8 1}$ & $\mathbf{1 0 2 . 9 9}$ & $\mathbf{6 7 . 8 7}$ & $\mathbf{7 6 . 0 1}$ & $\mathbf{6 2 . 5 7}$ & $\mathbf{1 6 2 . 7 9}$ \\
\hline
\end{tabular}

\section{Appendix B: Nonparametric TFP Decompositions when there are Many Outputs}

The analysis in this Appendix is an adaptation of the analysis presented in section 2 of Diewert (2014). We assume that we have information on the period t vector of net outputs produced by a production unit, $\mathrm{y}^{\mathrm{t}} \equiv\left[\mathrm{y}_{1}{ }^{\mathrm{t}}, \ldots, \mathrm{y}_{\mathrm{M}}{ }^{\mathrm{t}}\right]$ for $\mathrm{t}=1, \ldots, \mathrm{T}$ where the number of outputs is $\mathrm{M}$ $\geq 2 .{ }^{27}$ As in the main text, the vector of inputs used during period $t$ is $x^{t} \equiv\left[\mathrm{x}_{1}{ }^{t}, \ldots, \mathrm{x}_{N}{ }^{t}\right]>0_{N}$, and the corresponding vector of input prices is $\mathrm{w}^{\mathrm{t}} \equiv\left[\mathrm{w}_{1}{ }^{\mathrm{t}}, \ldots, \mathrm{w}_{\mathrm{N}}{ }^{\mathrm{t}}\right]>0_{\mathrm{N}}$ for periods $\mathrm{t}=1, \ldots, \mathrm{T}$. Again, the period $\mathrm{t}$ observed total input cost is defined as $\mathrm{C}^{\mathrm{t}} \equiv \mathrm{w}^{\mathrm{t}} \cdot \mathrm{x}^{\mathrm{t}}$ for $\mathrm{t}=1, \ldots, \mathrm{T}$.

We assume that the production unit's period t production possibilities set can be adequately approximated by the convex conical free disposal hull of the period $t$ actual production vector and past production vectors that are in our sample of time series observations for the unit. Using this assumption, for each vector of input prices $w>0_{N}$ and vector of feasible net outputs $\mathrm{y},{ }^{28}$ we define the period t best practice (total) cost function $\mathrm{C}(\mathrm{y}, \mathrm{w}, \mathrm{t})$, as follows for $\mathrm{t}$ $=1, \ldots, \mathrm{T}$ :

(B1) $\mathrm{C}(\mathrm{y}, \mathrm{w}, \mathrm{t}) \equiv \min _{\lambda_{1}, \ldots, \lambda_{t}}\left\{\mathrm{w} \cdot\left(\sum_{\mathrm{s}=1}{ }^{\mathrm{t}} \mathrm{x}_{\mathrm{s}} \lambda_{\mathrm{s}}\right) ; \sum_{\mathrm{s}=1}{ }^{\mathrm{t}} \mathrm{y}^{\mathrm{s}} \lambda_{\mathrm{s}} \geq \mathrm{y} ; \lambda_{1} \geq 0, \ldots, \lambda_{\mathrm{t}} \geq 0\right\}$.

It will not necessarily be the case that the production unit being studied achieves the best practice level of costs in period t; i.e., the following inequalities will be satisfied:

(B2) $\mathrm{C}^{\mathrm{t}}=\mathrm{w}^{\mathrm{t}} \cdot \mathrm{x}^{\mathrm{t}} \geq \mathrm{C}\left(\mathrm{y}^{\mathrm{t}}, \mathrm{w}^{\mathrm{t}}, \mathrm{t}\right)$;

$\mathrm{t}=1, \ldots, \mathrm{T}$.

Thus the observed period $t$ cost for the unit, $C^{t}$, will be equal to or greater than the best practice minimum cost, $\mathrm{C}\left(\mathrm{y}^{\mathrm{t}}, \mathrm{w}^{\mathrm{t}}, \mathrm{t}\right)$, where this minimum cost is computed using the period $\mathrm{t}$ best practice technology, the same vector of outputs $\mathrm{y}^{\mathrm{t}}$ that the unit produced during period $\mathrm{t}$ and facing the same input prices $\mathrm{w}^{\mathrm{t}}$ that the production unit faced during period $\mathrm{t}$. As in the main text, the difference between these two costs or their ratio can serve as a measure of the cost efficiency of the unit during period t. Thus again following Balk $(1998 ; 28)$, we define the cost efficiency of the production unit during period $\mathrm{t}, \mathrm{e}^{\mathrm{t}}$, as follows:

(B3) $\mathrm{e}^{\mathrm{t}} \equiv \mathrm{C}\left(\mathrm{y}^{\mathrm{t}}, \mathrm{w}^{\mathrm{t}}, \mathrm{t}\right) / \mathrm{w}^{\mathrm{t}} \cdot \mathrm{x}^{\mathrm{t}} \leq 1$;

$\mathrm{t}=1, \ldots, \mathrm{T}$

\footnotetext{
${ }^{27}$ If the mth net output is an intermediate input used during period $t$, then $\mathrm{y}_{\mathrm{m}}{ }^{\mathrm{t}}$ is equal to minus the quantity of this intermediate input that was used during the period.

${ }^{28}$ The net output vector $\mathrm{y}$ is a feasible net output vector if a feasible solution exists for the linear programming problem that is defined by (A1).
} 
where the inequalities in (B3) follow from (B2). Thus if the establishment or firm is cost efficient in period $\mathrm{t}, \mathrm{e}^{\mathrm{t}}$ will equal its upper bound of 1 . Given the above definition of cost efficiency in period $\mathrm{t}$, we can define an index of the change in the production unit's cost efficiency over the two periods as follows:

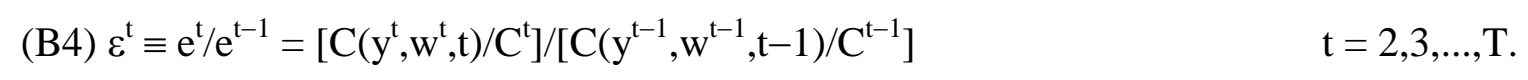

Thus if $\varepsilon^{t}>1$, then the cost efficiency of the production unit has improved going from period $\mathrm{t}-1$ to $\mathrm{t}$ whereas it has fallen if $\varepsilon^{\mathrm{t}}<1$.

At this point, we will follow the methodology that is used in the theoretical index number literature that originated with Konüs (1939) and Allen (1949) and we will use the reference cost function to define three families of indexes that vary only one of the three sets of variables, $\mathrm{t}, \mathrm{y}$ and $\mathrm{w}$, between the two periods under consideration and hold constant the other two sets of variables. ${ }^{29}$

Our first family of factors that explain cost changes is a family of cost based measures of output change, $\alpha\left(\mathrm{y}^{0}, \mathrm{y}^{1}, \mathrm{w}, \mathrm{s}\right)$ :

(B5) $\alpha\left(\mathrm{y}^{\mathrm{t}-1}, \mathrm{y}^{\mathrm{t}}, \mathrm{w}, \mathrm{s}\right) \equiv \mathrm{C}\left(\mathrm{y}^{\mathrm{t}}, \mathrm{w}, \mathrm{s}\right) / \mathrm{C}\left(\mathrm{y}^{\mathrm{t}-1}, \mathrm{w}, \mathrm{s}\right)$

For each choice of best practice technology for period s and for each choice of a reference vector of input prices $\mathrm{w}$, we obtain a measure of the effects on best practice cost of a change in output quantities going from period $t-1$ to $t$.

Following the example of Konüs (1939), it is natural to single out the following two special cases of the family of output quantity indexes defined by (B5):

(B6) $\alpha_{\mathrm{L}}{ }^{\mathrm{t}} \equiv \alpha\left(\mathrm{y}^{\mathrm{t}-1}, \mathrm{y}^{\mathrm{t}}, \mathrm{w}^{\mathrm{t}-1}, \mathrm{t}-1\right)=\mathrm{C}\left(\mathrm{y}^{\mathrm{t}}, \mathrm{w}^{\mathrm{t}-1}, \mathrm{t}-1\right) / \mathrm{C}\left(\mathrm{y}^{\mathrm{t}-1}, \mathrm{w}^{\mathrm{t}-1}, \mathrm{t}-1\right)$; $\mathrm{t}=2, \ldots, \mathrm{T}$

(B7) $\alpha_{P}{ }^{t} \equiv \alpha\left(y^{t-1}, y^{t}, w^{t}, t\right) \quad=C\left(y^{t}, w^{t}, t\right) / C\left(y^{t-1}, w^{t}, t\right)$; $\mathrm{t}=2, \ldots, \mathrm{T}$.

Since both measures of output change, $\alpha_{L}{ }^{t}$ and $\alpha_{P}{ }^{t}$, are equally representative, a single estimate of cost change due to output quantity changes between the two periods should be set equal to a symmetric average of these two estimates. We will choose the geometric mean as our preferred symmetric average and thus our preferred measure of cost based output quantity growth is the following Fisher type theoretical measure, $\alpha$ :

(B8) $\alpha^{t} \equiv\left[\alpha_{L}{ }^{t} \alpha_{P}^{t}\right]^{1 / 2}$ $\mathrm{t}=2, \ldots, \mathrm{T}$.

We now turn our attention to measures of the effects on best practice cost of input price change. We use the period s best practice cost function $\mathrm{C}(\mathrm{y}, \mathrm{w}, \mathrm{s})$ in order to define a family of input price indexes, $\beta\left(\mathrm{w}^{\mathrm{t}-1}, \mathrm{w}^{\mathrm{t}}, \mathrm{y}, \mathrm{s}\right)$, as follows:

\footnotetext{
${ }^{29}$ The theory which follows is adapted from Diewert (2014). This approach to the output quantity and input price indexes is a reasonably straightforward adaptation of the earlier work on theoretical price and quantity indexes by Konüs (1939), Allen (1949), Fisher and Shell (1972), Archibald (1977), Diewert (1980; 461) (1983; 1054-1083) (2011) (2012) and Balk (1998).
} 
(B9) $\beta\left(\mathrm{w}^{\mathrm{t}-1}, \mathrm{w}^{\mathrm{t}}, \mathrm{y}, \mathrm{s}\right) \equiv \mathrm{C}\left(\mathrm{y}, \mathrm{w}^{\mathrm{t}}, \mathrm{s}\right) / \mathrm{C}\left(\mathrm{y}, \mathrm{w}^{\mathrm{t}-1}, \mathrm{~s}\right)$.

Again following the example of Konüs (1939) in his analysis of the true cost of living index, it is natural to single out two special cases of the family of input price indexes defined by (B9): one choice where we use the period $\mathrm{t}-1$ technology and set the reference quantities equal to the period $\mathrm{t}-1$ quantities $\mathrm{y}^{\mathrm{t}-1}$ (which gives rise to a Laspeyres type input price index) and another choice where we use the period t technology and set the reference quantities equal to the period t quantities $\mathrm{y}^{\mathrm{t}}$ (which gives rise to a Paasche type input price index). Thus define these special cases as $\beta_{\mathrm{L}}{ }^{\mathrm{t}}$ and $\beta_{\mathrm{P}}{ }^{\mathrm{t}}$ :

(B10) $\beta_{\mathrm{L}}{ }^{\mathrm{t}} \equiv \beta\left(\mathrm{w}^{\mathrm{t}-1}, \mathrm{w}^{\mathrm{t}}, \mathrm{y}^{\mathrm{t}-1}, \mathrm{t}-1\right)=\mathrm{C}\left(\mathrm{y}^{\mathrm{t}-1}, \mathrm{w}^{\mathrm{t}}, \mathrm{t}-1\right) / \mathrm{C}\left(\mathrm{y}^{\mathrm{t}-1}, \mathrm{w}^{\mathrm{t}-1}, \mathrm{t}-1\right)$; $\mathrm{t}=2, \ldots, \mathrm{T}$

(B11) $\beta_{\mathrm{P}}{ }^{\mathrm{t}} \equiv \beta\left(\mathrm{w}^{\mathrm{t}-1}, \mathrm{w}^{\mathrm{t}}, \mathrm{y}^{\mathrm{t}}, \mathrm{t}\right)=\mathrm{C}\left(\mathrm{y}^{\mathrm{t}}, \mathrm{w}^{\mathrm{t}}, \mathrm{t}\right) / \mathrm{C}\left(\mathrm{y}^{\mathrm{t}}, \mathrm{w}^{\mathrm{t}-1}, \mathrm{t}\right)$; $\mathrm{t}=2, \ldots, \mathrm{T}$.

Since both input price indexes, $\beta_{\mathrm{L}}{ }^{\mathrm{t}}$ and $\beta_{\mathrm{P}}{ }^{\mathrm{t}}$, are equally representative, a single estimate of input price change should be set equal to a symmetric average of these two estimates. We again choose the geometric mean as our preferred symmetric average and thus our preferred overall measure of input price growth is the following Fisher type theoretical index, $\beta^{\mathrm{t}}$ :

(B12) $\beta^{\mathrm{t}} \equiv\left[\beta_{\mathrm{L}}^{\mathrm{t}} \beta_{\mathrm{P}}^{\mathrm{t}}\right]^{1 / 2}$ $\mathrm{t}=2, \ldots, \mathrm{T}$.

We now define our last family of cost function based indexes for this section. We again use reference total cost function defined by (B1) in order to define a family of indexes of technical progress going from period $\mathrm{t}-1$ to $\mathrm{t}, \tau(\mathrm{t}-1, \mathrm{t}, \mathrm{y}, \mathrm{w})$, for a reference vector of outputs $\mathrm{y}$ and a reference vector of input prices $\mathrm{w}$ as follows:

$$
\tau(\mathrm{t}-1, \mathrm{t}, \mathrm{y}, \mathrm{w}) \equiv \mathrm{C}(\mathrm{y}, \mathrm{w}, \mathrm{t}-1) / \mathrm{C}(\mathrm{y}, \mathrm{w}, \mathrm{t}) ; \quad \mathrm{t}=2, \ldots, \mathrm{T} \text {. }
$$

Technical progress measures are usually defined in terms of upward shifts in production functions or outward shifts of production possibilities sets due to the discovery of new techniques or managerial innovations over time. However, in the regulatory literature, it is quite common to specify technical progress in terms of downward shifts in the cost function over time. Thus in definition (B13), we pick reference vectors $\mathrm{y}$ and $\mathrm{w}$ and use the best practice technology of periods $t-1$ and $t$ to calculate the minimum cost of producing the output vector $\mathrm{y}$ at the input prices $\mathrm{w}$. This gives rise to the total costs, $\mathrm{C}(\mathrm{y}, \mathrm{w}, \mathrm{t}-1)$ and $\mathrm{C}(\mathrm{y}, \mathrm{w}, \mathrm{t})$, respectively. If there is positive technical progress going from period $t-1$ to $t$, then $C(y, w, t)$ will be less than $C(y, w, t-1)$ and hence $\tau(t-1, t, y, w)$ will be greater than one and this measure of technical progress is the reciprocal of the degree of proportional cost reduction that results from the expansion of the underlying best practice technology sets due to the passage of time. For each choice of a reference vector of output quantities y and reference vector of input prices $\mathrm{w}$, we obtain a possibly different measure of exogenous cost reduction and hence of technical progress.

Instead of singling out the reference vectors $\mathrm{y}$ and $\mathrm{w}$ that appear in the definition of $\tau(\mathrm{t}-1, \mathrm{t}, \mathrm{y}, \mathrm{w})$ to be the period $\mathrm{t}$ quantity and price vectors $\left(\mathrm{y}^{\mathrm{t}}, \mathrm{w}^{\mathrm{t}}\right)$, we will choose the mixed vectors $\left(\mathrm{y}^{0}, \mathrm{w}^{1}\right)$ and $\left(\mathrm{y}^{1}, \mathrm{w}^{0}\right)$ for special attention. The reason for these rather odd looking 
choices is that with these choices, we can obtain an exact decomposition of observed total cost into explanatory factors. ${ }^{30}$

B14) $\tau_{\mathrm{t}-1, \mathrm{t}}{ }^{\mathrm{t}} \equiv \tau\left(\mathrm{t}-1, \mathrm{t}, \mathrm{y}^{\mathrm{t}-1}, \mathrm{w}^{\mathrm{t}}\right)=\mathrm{C}\left(\mathrm{y}^{\mathrm{t}-1}, \mathrm{w}^{\mathrm{t}}, \mathrm{t}-1\right) / \mathrm{C}\left(\mathrm{y}^{\mathrm{t}-1}, \mathrm{w}^{\mathrm{t}}, \mathrm{t}\right)$;

$\mathrm{t}=2, \ldots, \mathrm{T}$

$\tau_{\mathrm{t}, \mathrm{t}-1}{ }^{\mathrm{t}} \equiv \tau\left(\mathrm{t}-1, \mathrm{t}, \mathrm{y}^{\mathrm{t}}, \mathrm{w}^{\mathrm{t}-1}\right)=\mathrm{C}\left(\mathrm{y}^{\mathrm{t}}, \mathrm{w}^{\mathrm{t}-1}, \mathrm{t}-1\right) / \mathrm{C}\left(\mathrm{y}^{\mathrm{t}}, \mathrm{w}^{\mathrm{t}-1}, \mathrm{t}\right)$;

$\mathrm{t}=2, \ldots, \mathrm{T}$.

Since both of the above measures of technical progress, $\tau_{t-1, t}{ }^{t}$ and $\tau_{t, t-1}{ }^{t}$, are equally representative, a single estimate of technical progress should be set equal to a symmetric average of these two estimates. We again choose the geometric mean as our preferred symmetric average ${ }^{31}$ and thus our preferred summary measure of technical progress going from period $t-1$ to $t$ is the following Fisher type index of technical progress, $\tau^{\mathrm{t}}$ :

(B16) $\tau^{\mathrm{t}} \equiv\left[\tau_{\mathrm{t}-1, \mathrm{t}}{ }^{\mathrm{t}} \tau_{\mathrm{t}, \mathrm{t}-1}{ }^{\mathrm{t}}\right]^{1 / 2}$

$\mathrm{t}=2, \ldots, \mathrm{T}$.

We want to explain the growth in total costs going from period $t-1$ to $t$ for the production unit under consideration, $\mathrm{C}^{\mathrm{t}} / \mathrm{C}^{\mathrm{t}-1}=\mathrm{w}^{\mathrm{t}} \cdot \mathrm{x}^{\mathrm{t}} / \mathrm{w}^{\mathrm{t}-1} \cdot \mathrm{x}^{\mathrm{t}-1}$, as the product of four growth factors:

- Growth in outputs; i.e., a factor of the form $\alpha\left(\mathrm{y}^{\mathrm{t}-1}, \mathrm{y}^{\mathrm{t}}, \mathrm{w}, \mathrm{s}\right)$ defined above by (B5);

- Growth in input prices; i.e., a factor of the form $\beta\left(\mathrm{w}^{\mathrm{t}-1}, \mathrm{w}^{\mathrm{t}}, \mathrm{y}, \mathrm{s}\right)$ defined by (B9);

- Exogenous reduction in costs due to technical progress; i.e., a factor of the form $\tau(\mathrm{t}-1, \mathrm{t}, \mathrm{y}, \mathrm{w})$ defined by (B13) and

- Changes in the production unit's cost efficiency over the two periods; i.e., the factor $\varepsilon^{\mathrm{t}}$ defined by (B4) above.

Using the algebra developed in Diewert (2014) and the above definitions, it can be shown we have the following decomposition of the observed cost ratio $\mathrm{C}^{\mathrm{t}} / \mathrm{C}^{\mathrm{t}-1}$ into explanatory factors of the above type:

(B17) $C^{t} / C^{t-1}=\alpha^{t} \beta^{t} / \tau^{t} \varepsilon^{t}$;

$\mathrm{t}=2, \ldots, \mathrm{T}$

where $\alpha^{\mathrm{t}}, \beta^{\mathrm{t}}, \tau^{\mathrm{t}}$ and $\varepsilon^{\mathrm{t}}$ are defined by (B8), (B12), (B16) and (B4) respectively. ${ }^{32}$

An (implicit) index of input growth going from period $\mathrm{t}-1$ to $\mathrm{t}, \gamma^{\mathrm{t}}$, can be defined as the cost ratio $\mathrm{C}^{\mathrm{t}} / \mathrm{C}^{\mathrm{t}-1}$ divided by the input price index $\beta^{\mathrm{t}}$ :

(B18) $\gamma^{\mathrm{t}} \equiv\left[\mathrm{C}^{\mathrm{t}} / \mathrm{C}^{\mathrm{t}-1}\right] / \beta^{\mathrm{t}}$

$\mathrm{t}=2, \ldots, \mathrm{T}$.

Total Factor Productivity growth going from period $\mathrm{t}-1$ to $\mathrm{t}$ can be defined as the index of output growth $\alpha^{t}$ divided by the index of input growth $\gamma^{t}$. Using this definition of TFP growth,

\footnotetext{
${ }^{30}$ With only one output, we did not have to use mixed vectors to define our measures of technical progress in the main text because with one output and constant returns to scale, the total cost function simplifies into the product of the single output level times the unit cost function.

${ }^{31}$ This will ensure that the resulting measure of technical progress satisfies the time reversal property; i.e., if we reverse the role of time and recalculate the measure of technical progress, we obtain the reciprocal of the original measure when we take the geometric average.

${ }^{32}$ Note that in order to define the growth factors $\alpha^{t}, \beta^{t}, \tau^{t}$ and $\varepsilon^{t}$, we need to solve 8 linear programming problems of the type defined by (B1) for each time period t.
} 
(B17) and (B18), it can be seen that we have the following exact decomposition of TFP growth into explanatory factors:

(B18) $\mathrm{TFP}_{\mathrm{G}}^{\mathrm{t}} \equiv \alpha^{\mathrm{t}} / \gamma^{\mathrm{t}}=\varepsilon^{\mathrm{t}} \tau^{\mathrm{t}}$

$$
\mathrm{t}=2, \ldots, \mathrm{T} \text {. }
$$

(B18) is the counterpart to (16) in the main text. Thus even in the case where there are many outputs and inputs, we can decompose TFP growth over two periods into the product of cost efficiency change, $\varepsilon^{\mathrm{t}}$, times an index of technical progress over the two periods, $\tau^{\mathrm{t}}{ }^{33}$

\section{References}

Aghion, P., and P. Howitt (1992), "A Model of Growth Through Creative Destruction. Econometrica 60, 323-51.

Aiyar, S., C-J. Dalgaard and O. Moav (2008), “Technological Progress and Regress in Preindustrial Times", Journal of Economic Growth 13, 125-144.

Allen, R.D.G. (1949), “The Economic Theory of Index Numbers”, Economica 16, 197-203.

Archibald, R.B. (1977), "On the Theory of Industrial Price Measurement: Output Price Indexes", Annals of Economic and Social Measurement 6, 57-62.

Balk, B.M. (1998), Industrial Price, Quantity and Productivity Indices, Boston: Kluwer Academic Publishers.

Byrne, D.M., J.G. Fernald and M.B. Reinsdorf (2016), "Does the United States Have a Productivity Slowdown or a Measurement Problem?" Federal Reserve Bank of San Francisco Working Paper 2016-03.

Christensen, L.R. and D.W. Jorgenson (1969), "The Measurement of U.S. Real Capital Input, 1929-1967", Review of Income and Wealth 15, 293-320.

Diamond, J. (1995), “Easter Island's End”, Discover Magazine.

Diewert, W.E. (1980a), “Aggregation Problems in the Measurement of Capital”, pp. 433-528 in The Measurement of Capital, D. Usher (ed.), Chicago: The University of Chicago Press.

Diewert, W.E. (1980b), "Capital and the Theory of Productivity Measurement", American Economic Review 70, 260-267.

Diewert, W.E. (1981), "The Theory of Total Factor Productivity Measurement in Regulated Industries", pp. 17-44 in Productivity Measurement in Regulated Industries, T. Cowing and R. Stevenson (eds.), New York: Academic Press.

\footnotetext{
${ }^{33}$ Equations (17)-(19) in the main text can be adapted to give a levels decomposition of TFP into explanatory factors in the many output case.
} 
Diewert, W.E. (1983), "The Theory of the Output Price Index and the Measurement of Real Output Change", pp. 1049-1113 in Price Level Measurement, W.E. Diewert and C. Montmarquette (eds.), Ottawa: Statistics Canada.

Diewert, W.E. (2011), "Measuring Productivity in the Public Sector: Some Conceptual Problems", Journal of Productivity Analysis 36, 177-191.

Diewert, W.E. (2012), “The Measurement of Productivity in the Nonmarket Sector", Journal of Productivity Analysis 37, 217-229.

Diewert, W.E. (2014), "Decompositions of Profitability Change using Cost Functions, Journal of Econometrics 183, 58-66.

Diewert, W.E. and K.J. Fox (2014), "Reference Technology Sets, Free Disposal Hulls and Productivity Decompositions", Economics Letters 122, 238-242.

Diewert, W.E. and K.J. Fox (2016a), "Decomposing Productivity Indexes into Explanatory Factors", European Journal of Operational Research, forthcoming.

Diewert, W.E and K.J. Fox (2016b), "Alternative User Costs, Rates of Return and TFP Growth Rates for the US Nonfinancial Corporate and Noncorporate Business Sectors: 1960-2014", Discussion Paper 16-03, Vancouver School of Economics, The University of British Columbia, Vancouver, Canada, V6T 1L4.

Diewert, W.E. and C. Parkan (1983), "Linear Programming Tests of Regularity Conditions for Production Functions", pp. 131-158 in Quantitative Studies on Production and Prices, W. Eichhorn, R. Henn, K. Neumann and R.W. Shephard (eds.), Vienna: Physica-Verlag.

Eaton, J., and S. Kortum (1999), "International Technology Diffusion: Theory and measurement", International Economic Review 40, 537-70.

Färe, R., S. Grosskopf, M. Norris and Z. Zhang (1994), "Productivity Growth, Technical Progress, and Efficiency Change in Industrialized Countries", American Economic Review 84, 66-83.

Farrell, M.J. (1957), "The Measurement of Production Efficiency", Journal of the Royal Statistical Society, Series A, 120, 253-278.

Fisher, I. (1922), The Making of Index Numbers, Boston: Houghton-Mifflin.

Fisher, F.M. and K. Shell (1972), “The Pure Theory of the National Output Deflator”, pp. 49113 in The Economic Theory of Price Indexes, New York: Academic Press.

Fox, K.J. and U. Kohli (1998), "GDP Growth, Terms of Trade Effects and Total Factor Productivity", Journal of International Trade and Development Economics 7, 87-110.

Grossman, G., and E. Helpman (1991), Innovation and Growth in the Global Economy, Cambridge: MIT Press. 
Gordon, R.J. (2012), "Is the U.S. Economic Growth Over? Faltering Innovation Confronts the Six Headwinds", National Bureau of Economic Research Working Paper 18315.

Jorgenson, D.W. and Z. Griliches (1967), The Explanation of Productivity Change", The Review of Economic Studies 34, 249-283.

Kohli, U. (1990), "Growth Accounting in the Open Economy: Parametric and Nonparametric Estimates”, Journal of Economic and Social Measurement 16, 125-136.

Konüs, A.A. (1939), "The Problem of the True Index of the Cost of Living”, Econometrica 7 , 10-29.

Kortum, S. (1997), "Research, patenting, and technological change”, Econometrica 65, 13891419.

Nelson, R. R. and E.S. Phelps (1966), "Investment in Humans, Technological Diffusion, and Economic Growth", American Economic Review 56, 69-75.

Olsson, O. (2000), "Knowledge as a Set in Idea Space: An epistemological view on growth," Journal of Economic Growth 5, 253-275.

Romer, P. (1990), "Endogenous technological change", Journal of Political Economy 98, S71-S102.

Salter, W. E. G. (1960), Productivity and Technical Change, Cambridge U.K.: Cambridge University Press.

Shephard, R.W. (1953), Cost and Production Functions, Princeton N.J.: Princeton University Press.

Syverson, C. (2011), “What Determines Productivity?” Journal of Economic Literature 49, 326-365.

Syverson, C. (2016), "Challenges to Mismeasurement Explanations for the U.S. Productivity Slowdown”, National Bureau of Economic Research Working Paper 21974.

Tulkens, H. (1986), "La Performance Productive d'un Service Public”, L'Actualité Economique 62, 306-335.

Tulkens, H. (1993), “On FDH Efficiency Analysis: Some Methodological Issues and Application to Retail Banking, Courts, and Urban Transit", Journal of Productivity Analysis 4, 183-210.

Weitzman, M. (1998), “Recombinant Growth”, Quarterly Journal of Economics 113, 331360.

Wilson, A. (2002), "Machines, Power and the Ancient Economy", Journal of Roman Studies 92, 1-32. 